\title{
Copyright
}

by

Shankar Mahadevan

2010 
The Dissertation Committee for Shankar Mahadevan certifies that this is the approved version of the following dissertation:

\section{Simulation of Direct-Current Surface Plasma Discharges in Air for Supersonic Flow Control}

Committee:

Laxminarayan L. Raja, Supervisor

Philip L. Varghese

Graham F. Carey

Gary A. Hallock

Ofodike A. Ezekoye 


\title{
Simulation of Direct-Current Surface Plasma Discharges in Air for Supersonic Flow Control
}

\author{
by
}

Shankar Mahadevan, B.Tech., M.S.E

\author{
Dissertation \\ Presented to the Faculty of the Graduate School of \\ The University of Texas at Austin \\ in Partial Fulfillment \\ of the Requirements \\ for the Degree of \\ Doctor Of Philosophy
}

The University Of Texas At Austin

May 2010 


\section{Acknowledgments}

There are many people who have contributed directly and indirectly to the completion of this dissertation, and to whom I owe a debt of extreme gratitude. My deepest gratitude is to my advisor, Dr. Laxminarayan Raja. His guidance and support have been instrumental in enabling me to complete my dissertation. I am thankful to Dr. Philip Varghese, Dr. Gary Hallock, Dr. Ofodike Ezekoye and Dr. Graham Carey for being on my dissertation committee, and for their insights, suggestions and advice that has led to the improvement of this manuscript.

I thank my past and present colleagues in the Plasma Research Laboratory: Xiaohui Yuan, Prashanth Kothnur, Jichul Shin, Guangye Chen, Thomas Deconinck, Utsav KC, Marshall Albright, Doug Breden, Hariswaran Sitaraman, and Timothy Min. I would also like to thank Donna Soward at the Center for Aeromechanics Research and Scott Messec, the manager of computing services in the Aerospace Engineering and Engineering Mechanics department for all their help.

I am grateful for the financial support I have received from the AFOSRMURI program (grant FA 9550-04-1-0387) during the course of my graduate studies. I would like to acknowledge the Texas Advanced Computing Center (TACC) for their High Performance Computing resources, and Dr. Chad Lan-

dis from the Aerospace Engineering and Engineering Mechanics department 
who graciously allowed me to use his Linux HPC cluster in the later stages of this research.

Finally, I would like to thank my family for all their support and encouragement. 


\title{
Simulation of Direct-Current Surface Plasma Discharges in Air for Supersonic Flow Control
}

\author{
Shankar Mahadevan, Ph.D. \\ The University of Texas at Austin, 2010
}

Supervisor: Laxminarayan L. Raja

\begin{abstract}
Computational simulations of air glow discharge plasma in the presence of supersonic flow are presented. The glow discharge model is based on a self-consistent, multi-species, continuum description of the plasma with finiterate chemistry effects. The glow discharge model is coupled to a compressible Navier-Stokes solver to study the effect of the plasma on the flow and the counter-effect of the flow on the plasma. A finite-rate air chemistry model is presented and validated against experiments from the literature at a pressure of 600 mTorr. Computational results are compared with experimentally measured V-I characteristics, axial positive ion densities and electron temperature, and reasonably good qualitative and quantitative agreement is observed. The validated air plasma model is then used to study the effect of the surface plasma discharge on $\mathrm{M}=3$ supersonic flow at freestream pressure 18 Torr and the corresponding effects of the flow on the discharge structure in two dimensions. The species concentrations and the gas temperature are examined in the absence and presence of bulk supersonic flow. The peak gas temperature from the computations is found to be $1180 \mathrm{~K}$ with the surface plasma alone in the
\end{abstract}


absence of flow, and $830 \mathrm{~K}$ in the presence of supersonic flow. Results indicate that $\mathrm{O}^{-}$ions can have comparable densities to electrons in the pressure range 1-20 Torr, and that $\mathrm{O}_{2}^{-}$ion densities are at least two orders of magnitude smaller over the pressure range considered. Different ion species are found to be dominant in the absence and presence of supersonic flow, highlighting the importance of including finite-rate chemistry effects in discharge models for understanding plasma actuator physical phenomena. Electrode polarity effects are investigated, and the cathode upstream actuation is found to be stronger than the actuation strength with the cathode downstream, which is consistent with experimental findings of several groups. A parallel computing implementation of the plasma and flow simulation tools has been developed and is used to study the three-dimensional plasma actuator configuration with circular pin electrodes. 


\section{Table of Contents}

Acknowledgments iv

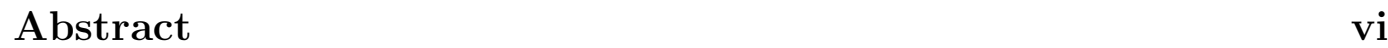

List of Tables $\quad$ x

List of Figures $\quad$ xii

Chapter 1. Introduction 1

1.1 Literature review . . . . . . . . . . . . . . . . 6

1.1.1 Surface-based approaches to high speed flow control . . 6

1.1.2 Continuum models of plasma discharges . . . . . . . 8

1.2 Objectives and methodology . . . . . . . . . . . . . . . . 11

1.3 Contributions of the present research . . . . . . . . . . 13

$\begin{array}{llr}\text { Chapter 2. } & \text { Model Description } & 14\end{array}$

2.1 Plasma Model . . . . . . . . . . . . . . . . . . . 14

2.1.1 Governing Equations . . . . . . . . . . . . . . 14

2.1.2 Boundary conditions . . . . . . . . . . . . . . . . 18

2.1.2.1 Species continuity boundary conditions . . . . . 19

2.1.2.2 Potential boundary conditions . . . . . . . . . 20

2.1.2.3 Electron and gas energy boundary conditions . 21

2.1.3 Spatial and temporal discretization . . . . . . . . 22

2.2 Navier-Stokes Solver . . . . . . . . . . . . . . . . . 27

2.2.1 Governing equations and boundary conditions . . . . . . 27

2.2.2 Spatial and temporal discretization . . . . . . . . . 28

2.3 Coupled plasma-compressible flow solution methodology . . . 29

$\begin{array}{lll}\text { Chapter 3. Air Plasma Discharge Validation } & 31\end{array}$

3.1 Air plasma chemistry and transport . . . . . . . . . . . . . . 31

3.2 Air plasma model validation results . . . . . . . . . . . . 38 
Chapter 4. Two-Dimensional Surface Plasma Actuator Study 56

4.1 Air surface plasma discharge in the absence of supersonic flow 59

4.2 Air surface plasma discharge in the presence of supersonic flow 63

4.3 Effect of electrode polarity . . . . . . . . . . . . . 75

Chapter 5. Development of a Parallel Computational Tool for Plasma and Flow Simulations $\quad 81$

5.1 Implementation . . . . . . . . . . . . . . . 82

5.2 Verification of the parallelized models and scalability . . . . . 87

Chapter 6. Three-Dimensional Pin Actuator Study 96

$\begin{array}{lll}\text { Chapter 7. Conclusion } & 106\end{array}$

$\begin{array}{ll}\text { Appendices } & 110\end{array}$

Appendix A. Electron impact excitation, ionization and attachment rate coefficients

Appendix B. Cross section data for electron impact collisions with $\mathrm{N}_{2}$ and $\mathrm{O}_{2}$

114

Bibliography

135

Vita

146 


\section{List of Tables}

3.1 Air plasma gas phase reaction mechanism. . . . . . . . . . . . 32

3.2 Air plasma surface reactions. . . . . . . . . . . . . . . 35

3.3 Neutral species transport properties. . . . . . . . . . . . . . . 36

3.4 Summary of species transport property data. . . . . . . . . . . 38

B.1 Cross section data for $\mathrm{N}_{2}$ Elastic $\left(\Delta E^{e}=1.95 e-005 \mathrm{eV}\right)$. . . 114

B.2 Cross section data for $\mathrm{N}_{2}$ Excitation $\left(\Delta E^{e}=0.02 \mathrm{eV}\right)$. . . . . 116

B.3 Cross section data for $\mathrm{N}_{2}$ Excitation $\left(\Delta E^{e}=0.291 \mathrm{eV}\right)$. . . . 116

B.4 Cross section data for $\mathrm{N}_{2}$ Excitation $\left(\Delta E^{e}=0.59 \mathrm{eV}\right)$. . . . 117

B.5 Cross section data for $\mathrm{N}_{2}$ Excitation $\left(\Delta E^{e}=0.88 \mathrm{eV}\right)$. . . . 117

B.6 Cross section data for $\mathrm{N}_{2}$ Excitation $\left(\Delta E^{e}=1.17 \mathrm{eV}\right)$. . . . . 118

B.7 Cross section data for $\mathrm{N}_{2}$ Excitation $\left(\Delta E^{e}=1.47 \mathrm{eV}\right)$. . . . 118

B.8 Cross section data for $\mathrm{N}_{2}$ Excitation $\left(\Delta E^{e}=1.76 \mathrm{eV}\right)$. . . . . 119

B.9 Cross section data for $\mathrm{N}_{2}$ Excitation $\left(\Delta E^{e}=2.06 \mathrm{eV}\right)$. . . . . 119

B.10 Cross section data for $\mathrm{N}_{2}$ Excitation $\left(\Delta E^{e}=2.35 \mathrm{eV}\right)$. . . . . 120

B.11 Cross section data for $\mathrm{N}_{2}$ Excitation $\left(\Delta E^{e}=6.17 \mathrm{eV}\right)$. . . . . 120

B.12 Cross section data for $\mathrm{N}_{2}$ Excitation $\left(\Delta E^{e}=7.35 \mathrm{eV}\right)$. . . . . 121

B.13 Cross section data for $\mathrm{N}_{2}$ Excitation $\left(\Delta E^{e}=7.36 \mathrm{eV}\right)$. . . . . 121

B.14 Cross section data for $\mathrm{N}_{2}$ Excitation $\left(\Delta E^{e}=8.16 \mathrm{eV}\right)$. . . . . 122

B.15 Cross section data for $\mathrm{N}_{2}$ Excitation $\left(\Delta E^{e}=8.4 \mathrm{eV}\right)$. . . . . 123

B.16 Cross section data for $\mathrm{N}_{2}$ Excitation $\left(\Delta E^{e}=8.55 \mathrm{eV}\right)$. . . 123

B.17 Cross section data for $\mathrm{N}_{2}$ Excitation $\left(\Delta E^{e}=8.89 \mathrm{eV}\right)$. . . . 124

B.18 Cross section data for $\mathrm{N}_{2}$ Excitation $\left(\Delta E^{e}=11.03 \mathrm{eV}\right)$. . . . 124

B.19 Cross section data for $\mathrm{N}_{2}$ Excitation $\left(\Delta E^{e}=11.88 \mathrm{eV}\right)$. . . . 125

B.20 Cross section data for $\mathrm{N}_{2}$ Excitation $\left(\Delta E^{e}=12.25 \mathrm{eV}\right)$. . . . 125

B.21 Cross section data for $\mathrm{N}_{2}$ Excitation $\left(\Delta E^{e}=13.0 \mathrm{eV}\right)$. . . . . 126

B.22 Cross section data for $\mathrm{N}_{2}$ Ionization $\left(\Delta E^{e}=15.6 \mathrm{eV}\right)$. . . . 126

B.23 Cross section data for $\mathrm{O}_{2}$ Elastic $\left(\Delta E^{e}=1.7 e-005 \mathrm{eV}\right) \quad$. . 127

B.24 Cross section data for $\mathrm{O}_{2}$ Excitation $\left(\Delta E^{e}=0.02 \mathrm{eV}\right)$. . . . 128 
B.25 Cross section data for $\mathrm{O}_{2}$ Excitation $\left(\Delta E^{e}=0.193 \mathrm{eV}\right) \quad \ldots \quad 129$

B.26 Cross section data for $\mathrm{O}_{2}$ Excitation $\left(\Delta E^{e}=0.98 \mathrm{eV}\right) \ldots \ldots .131$

B.27 Cross section data for $\mathrm{O}_{2}$ Excitation $\left(\Delta E^{e}=1.63 \mathrm{eV}\right) \ldots \ldots .131$

B.28 Cross section data for $\mathrm{O}_{2}$ Excitation $\left(\Delta E^{e}=4.5 \mathrm{eV}\right) \ldots \ldots$

B.29 Cross section data for $\mathrm{O}_{2}$ Excitation $\left(\Delta E^{e}=5.58 \mathrm{eV}\right) \ldots \ldots .132$

B.30 Cross section data for $\mathrm{O}_{2}$ Excitation $\left(\Delta E^{e}=8.4 \mathrm{eV}\right) \ldots \ldots 133$

B.31 Cross section data for $\mathrm{O}_{2}$ Attachment $\left(\Delta E^{e}=3.6 \mathrm{eV}\right) \quad \ldots \quad 133$

B.32 Cross section data for $\mathrm{O}_{2}$ Ionization $\left(\Delta E^{e}=12.07 \mathrm{eV}\right) \quad \ldots \quad 133$ 


\section{List of Figures}

1.1 Schematic of the surface plasma actuator configuration used in the present study showing the various actuation mechanisms. .

2.1 Equations solved in the plasma model in a given time step. . . 26

2.2 Flowchart of the combined plasma-flow model. . . . . . . . . . 30

3.1 Electron impact reaction rate coefficients . . . . . . . . . . 33

3.2 Mobility data for electrons . . . . . . . . . . . . . . . 36

3.3 Mobility data for ions . . . . . . . . . . . . . . . 37

3.4 Geometry and mesh used for the validation of the air plasma model. The mesh contains a total of 10160 cells, with 8000 cells in the plasma subdomain. . . . . . . . . . . . . . .

3.5 Comparison of current-voltage characteristic with experimental data. The pressure is 600 mTorr, and inter-electrode distance is $32 \mathrm{~mm}$. . . . . . . . . . . . . . . . .

3.6 Comparison of current-voltage characteristic with experimental data with various values of secondary electron emission coefficient for $\mathrm{N}_{2}^{+}$and $\mathrm{O}_{2}^{+}$. The pressure is $600 \mathrm{mTorr}$, and interelectrode distance is $32 \mathrm{~mm}$. . . . . . . . . . . . . . . . .

3.7 Comparison of the computed axial electron temperature (units: $\mathrm{K})$ with experimental data. The cathode is at $\mathrm{y}=0$ and the anode is at $\mathrm{y}=32 \mathrm{~mm}$. The applied voltage at the cathode is -408 V. . . . . . . . . . . . . . . . . . . . . . .

3.8 Comparison of the computed axial total positive ion density (units: $\mathrm{m}^{-3}$ ) with experimental data. The cathode is at $\mathrm{y}=$ 0 and the anode is at $\mathrm{y}=32 \mathrm{~mm}$. The applied voltage at the cathode is $-408 \mathrm{~V}$. . . . . . . . . . . . . . . . .

3.9 Number densities (units: $\mathrm{m}^{-3}$ ) of charged species in the air plasma model along the axis. The cathode is at $\mathrm{y}=0$ and the anode is at $\mathrm{y}=32 \mathrm{~mm}$. The applied voltage at the cathode is -408 V. . . . . . . . . . . . . . . . . . . . . . . .

3.10 Profiles of positive ion, electron, and negative ion density in the air plasma model along the discharge axis. The regions of the plasma are labeled: (1) cathode sheath, (2) electron-positive ion layer, (3) electron-positive ion-negative ion core and (4) anode sheath. The net charged species density is also shown. . . . . . 
3.11 Potential (units: V) profile in the air plasma model along the discharge axis. The cathode is at $\mathrm{y}=0$ and the anode is at $\mathrm{y}$ $=32 \mathrm{~mm}$. The applied voltage at the cathode is $-408 \mathrm{~V}$. . . .

3.12 Number density contours (units: $\mathrm{m}^{-3}$ ) of dominant negatively charged species (left: $\mathrm{O}^{-}$, right: $\mathrm{e}^{-}$) in the plasma subdomain. The applied voltage at the cathode is $-408 \mathrm{~V}$. . . . . . . . . .

3.13 Number density contours (units: $\mathrm{m}^{-3}$ ) of dominant positively charged species (left: $\mathrm{O}_{2}^{+}$, right: $\mathrm{N}_{2}^{+}$) in the plasma subdomain. The applied voltage at the cathode is $-408 \mathrm{~V}$. . . . . . . . . .

3.14 Contours of potential (units: V) in the plasma, quartz and outside subdomains and electron temperature (units: eV) in the plasma subdomain. . . . . . . . . . . . . . . .

3.15 Net surface charge density (units: $\mathrm{C} / \mathrm{m}^{2}$ ) on the plasma-quartz dielectric interface. . . . . . . . . . . . . .

3.16 Potential (units: V) and electron density (units: $\mathrm{m}^{-3}$ ) profiles in the air plasma model along the discharge axis for varying mesh resolutions in the plasma subdomain. The cathode is at $\mathrm{y}=0$ and the anode is at $\mathrm{y}=32 \mathrm{~mm}$. The applied voltage at the cathode is $-408 \mathrm{~V}$.

4.1 Computational domain and mesh for the plane plasma actuator simulations. The mesh contains 8504 cells, with the plasma model solved on the inset subdomain (in red) and the NavierStokes equations solved on the entire domain. . . . . . . . .

4.2 Computational mesh in the inset subdomain showing a schematic of the plane plasma actuator. The surface is modeled as a dielectric, with metal electrodes exposed to the incoming supersonic flow. The source voltage is applied at the cathode, with a resistance in series. . . . . . . . . . . . . . . . .

4.3 Species number densities (units: $\mathrm{m}^{-3}$ ) of (a) $\mathrm{e}^{-}$, and (b) $\mathrm{O}^{-}$ions in the surface plasma discharge (no bulk flow). The cathode source voltage is $-1000 \mathrm{~V}$, and the ambient pressure is fixed at 18 Torr. . . . . . . . . . . . . . .

4.4 Species number densities (units: $\mathrm{m}^{-3}$ ) of (a) $\mathrm{N}_{2}^{+}$, and (b) $\mathrm{O}_{2}^{+}$ ions in the surface plasma discharge (no bulk flow). The cathode source voltage is $-1000 \mathrm{~V}$, and the ambient pressure is fixed at 18 Torr.

Species number densities (units: $\mathrm{m}^{-3}$ ) of (a) $\mathrm{N}_{4}^{+}$, and (b) $\mathrm{O}_{4}^{+}$ ions in the surface plasma discharge (no bulk flow). The cathode source voltage is $-1000 \mathrm{~V}$, and the ambient pressure is fixed at 18 Torr. . . . . . . . . . . . . . . 
4.6 Gas Temperature contours (units: $\mathrm{K}$ ) in the surface plasma discharge (no bulk flow). The cathode source voltage is -1000 $\mathrm{V}$, and the ambient pressure is fixed at 18 Torr. . . . . . . . .

4.7 Species number densities (units: $\mathrm{m}^{-3}$ ) of (a) $\mathrm{e}^{-}$, and (b) $\mathrm{O}^{-}$ions in the presence of supersonic $M=3$ flow. The cathode source voltage is $-1000 \mathrm{~V}$, and the freestream pressure is 18 Torr. . .

4.8 Species number densities (units: $\mathrm{m}^{-3}$ ) of (a) $\mathrm{N}_{2}^{+}$, and (b) $\mathrm{O}_{2}^{+}$ ions in the presence of supersonic $\mathrm{M}=3$ flow. The cathode source voltage is $-1000 \mathrm{~V}$, and the freestream pressure is 18 Torr. . .

4.9 Species number densities (units: $\mathrm{m}^{-3}$ ) of (a) $\mathrm{N}_{4}^{+}$, and (b) $\mathrm{O}_{4}^{+}$ ions in the presence of supersonic $\mathrm{M}=3$ flow. The cathode source voltage is $-1000 \mathrm{~V}$, and the freestream pressure is 18 Torr. . .

4.10 Gas temperature contours (units: K) in the supersonic flow with the plasma (a) off and (b) on. The wall temperature is fixed at $300 \mathrm{~K}$, and the freestream temperature is $110 \mathrm{~K}$. Velocity streamlines in the boundary layer are also shown. . . . . . . .

4.11 Gas heating source terms (units: $\mathrm{W} / \mathrm{m}^{3}$ ) in the plasma with supersonic flow, showing the contributions of ion Joule heating, inelastic and elastic heating sources to the overall gas heating.

4.12 Pressure contours (units: $\mathrm{Pa}$ ) in the supersonic flow with the plasma (a) off and (b) on on the enitre flow domain. . . . . . .

4.13 (a) Effect of voltage on the electron density contours at fixed pressure (18 Torr) and (b) corresponding V-I curve. . . . . . .

4.14 Effect of freestream pressure on (a) the electron density contours (units: $\mathrm{m}^{-3}$ ) and (b) discharge input power and peak gas temperature. . . . . . . . . . . . .

4.15 Sensitivity of gas temperature in the plasma with supersonic flow to the ion Joule heating thermalization factor $\eta_{\mathrm{Th}}$. . . . .

4.16 Species number densities (units: $\mathrm{m}^{-3}$ ) of (a) $\mathrm{e}^{-}$, and (b) $\mathrm{O}^{-}$ ions with the cathode located downstream with respect to the supersonic flow. The cathode source voltage is $-1000 \mathrm{~V}$, and the freestream pressure is 18 Torr. . . . . . . . . . . . .

4.17 Species number densities (units: $\mathrm{m}^{-3}$ ) of (a) $\mathrm{N}_{2}^{+}$, and (b) $\mathrm{O}_{2}^{+}$ ions with the cathode located downstream with respect to the supersonic flow. The cathode source voltage is $-1000 \mathrm{~V}$, and the freestream pressure is 18 Torr. . . . . . . . . . . . . . .

4.18 Species number densities (units: $\mathrm{m}^{-3}$ ) of (a) $\mathrm{N}_{4}^{+}$, and (b) $\mathrm{O}_{4}^{+}$ ions with the cathode located downstream with respect to the supersonic flow. The cathode source voltage is $-1000 \mathrm{~V}$, and the freestream pressure is 18 Torr. . . . . . . . . . . . . 
4.19 (a) Gas temperature (units: K) and (b) pressure contours (units: $\mathrm{Pa}$ ) in the supersonic flow with the cathode located downstream with respect to the supersonic flow. . . . . . . . . . . .

5.1 Parallel algorithm to calculate fluxes at faces and matrix contributions at cells adjacent to faces. The unshaded boxes represent the serial implementation, and the shaded boxes denote additions to the serial implementation. . . . . . . . . . .

5.2 Schematic and mesh of the GEC RF reference cell. The mesh shown has 13170 cells, and is divided into 16 partitions using METIS.

5.3 Plot of the electron number density (units: $\mathrm{m}^{-3}$ ) on the axis at the midpoint between the electrodes as a function of the number of electron time steps on $\mathrm{N}=1,2,4$ and 8 processors. . . . .

5.4 Strong scaling curve for the GEC RF reference cell with 13,170 cells on 1-16 processors. . . . . . . . . . . . . . . . . . . . . .

5.5 Plot of the cathode current (units: $\mathrm{mA}$ ) as a function of the number of electron time steps on $\mathrm{N}=1,8$ processors. . . . . .

5.6 (a) Unweighted and (b) Weighted cell partitioning of the air plasma validation mesh into 8 partitions. The mesh contains 22920 cells.

5.7 Strong scaling curves for the air plasma validation case with 22,920 cells on $1-8$ processors. . . . . . . . . . . . . . . . . .

5.8 Weighted cell partitioning of the two-dimensional actuator mesh on 8 processors. The mesh contains 8504 cells with 4127 cells in the inset (plasma+flow) subdomain. . . . . . . . . . .

5.9 Strong scaling curve for the two-dimensional actuator case with 8504 cells on 1-8 processors. . . . . . . . . . . . . . . .

6.1 The circular pin-electrode actuator schematic is shown. The source voltage is applied at the cathode $(\mathrm{C})$, with a resistance in series. The anode $(\mathrm{A})$ is grounded. . . . . . . . . . . . .

6.2 The computational mesh used for the three-dimensional actuator simulations, divided into 128 partitions. The mesh contains a total of 343,010 cells. . . . . . . . . . . . . . . . . . . . . . .

6.3 Strong scaling curve for the three-dimensional actuator simulations with 343,010 cells on 1-128 processors. . . . . . . . . . .

6.4 Three-dimensional structure of the discharge in the pin-electrode configuration. The isosurface of $\mathrm{O}_{2}^{+}$at a value of $3 \times 10^{18} \mathrm{~m}^{-3}$ is shown. Contours of the potential (units: V) on the electrodes and the dielectric surface are also shown. 
6.5 Species number densities (units: $\mathrm{m}^{-3}$ ) of (a) $\mathrm{e}^{-}$, and (b) $\mathrm{O}^{-}$ ions in the surface plasma discharge (no bulk flow) in the pinelectrode configuration. The cathode source voltage is $-1000 \mathrm{~V}$, and the ambient pressure is fixed at 18 Torr. . . . . . . . . . . 102

6.6 Species number densities (units: $\mathrm{m}^{-3}$ ) of (a) $\mathrm{N}_{2}^{+}$, and (b) $\mathrm{O}_{2}^{+}$ ions in the surface plasma discharge (no bulk flow) in the pinelectrode configuration. The cathode source voltage is $-1000 \mathrm{~V}$, and the ambient pressure is fixed at 18 Torr. . . . . . . . . . . 103

6.7 Species number densities (units: $\mathrm{m}^{-3}$ ) of (a) $\mathrm{N}_{4}^{+}$, and (b) $\mathrm{O}_{4}^{+}$ ions in the surface plasma discharge (no bulk flow) in the pinelectrode configuration. The cathode source voltage is $-1000 \mathrm{~V}$, and the ambient pressure is fixed at 18 Torr. . . . . . . . .

6.8 Gas temperature (units: K) in the surface plasma discharge (no bulk flow) on the center-line in the pin-electrode configuration. The cathode source voltage is $-1000 \mathrm{~V}$, and the ambient pressure is fixed at 18 Torr. . . . . . . . . . . . . . . . 


\section{Chapter 1}

\section{Introduction}

Weakly ionized plasma discharges have a wide variety of applications in semiconductor processing [1], plasma displays, ozone generation [2], lamps,

lasers, environmental and biomedical applications [3], propulsion and aerodynamic flow control [4]. Weakly ionized plasmas are operated using directcurrent, radio-frequency, inductively-coupled, microwave and other sources that are characterized by ionization fractions of $10^{-7}-10^{-4}$. Under most operating conditions (currents $\leq 100 \mathrm{~mA}$ ) these plasmas are in thermal nonequilibrium since the temperature of the electrons $(\sim 1-10 \mathrm{eV})$ greatly exceeds the temperature of the ions and neutral species that remain close to room temperature $(\sim 300-1000 \mathrm{~K})$. They are hence referred to as non-thermal or "cold" plasmas.

In their simplest form, direct-current discharges are generated by applying a potential difference between parallel electrodes. Beyond a certain threshold known as the breakdown voltage, a self-sustained plasma is established between the electrodes. The bulk of the plasma is quasi-neutral, i.e. charge densities of positive charge carriers (positive ions) are equal to the charge densities of the negative charge carriers (electrons and negative ions, if any) in the plasma. However, in the region adjacent to the electrodes, a thin 
non-neutral region known as the sheath is formed. The primary function of the sheath is to maintain overall current constraints in the discharge by limiting the flux of highly mobile electrons to the electrode surfaces, resulting in a potential profile that accelerates positive ions towards the electrodes. When the positive ions impact the electrodes, secondary electrons are emitted from the metal surface and these electrons rapidly gain energy in the sheath sufficient to cause further ionization of the gas at the sheath edge. Hence, secondary electron emission is a key mechanism that helps establish a self-sustaining plasma for non-equilibrium direct-current discharges. At very low currents $(\sim$ $1 \mu \mathrm{A})$, a non-luminous plasma known as a "dark" or Townsend discharge is formed. As the current is increased to the order of 1-10 $\mathrm{mA}$, the plasma becomes luminous and exists in the so-called "glow" mode. Further increase of the discharge current to $\sim 100$ 's mA leads to the formation of the arc discharge. Arc discharges are thermal plasmas where the temperatures of the ions and neutrals (5000-10000 K) are comparable to the electron temperatures.

In recent years, there have been a significant number of computational and experimental studies investigating the application of plasma discharges as actuators for high speed flow control [4, 5, 6, 7]. Plasma actuators potentially offer important advantages such as sub-millisecond actuation time-scales, and the absence of moving mechanical parts. Non-equilibrium glow discharges are particularly attractive for flow actuation since they have relatively low power requirements compared to other surface discharges such as thermal arcs. 


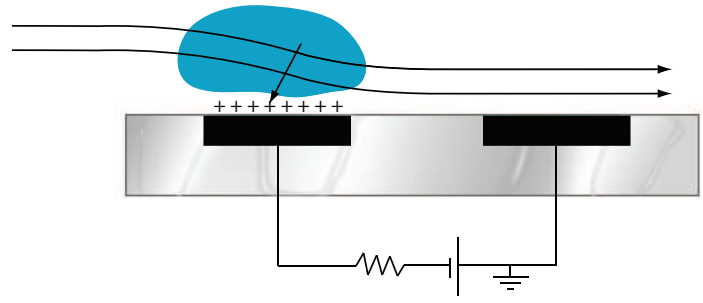

(a) Electrostatic forcing

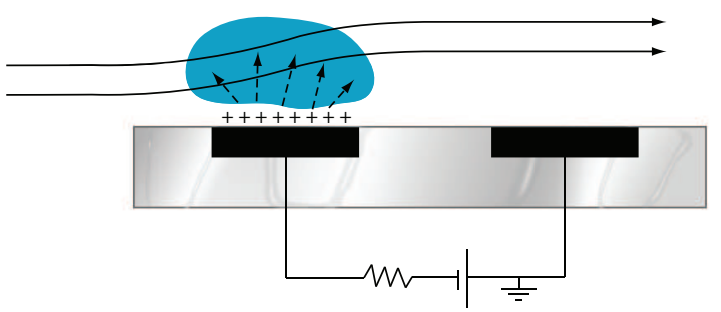

(b) Volumetric and surface heating

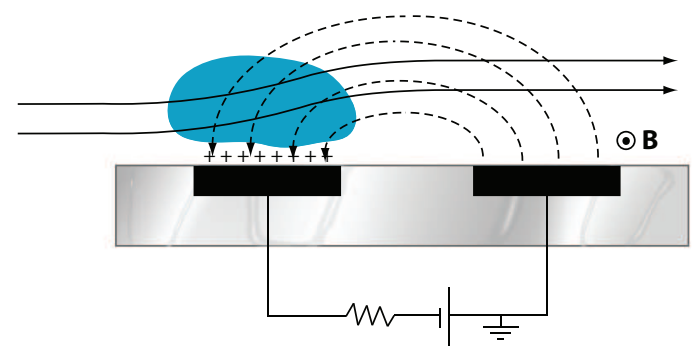

(c) Lorentz forcing

Figure 1.1: Schematic of the surface plasma actuator configuration used in the present study showing the various actuation mechanisms. The effect of the actuation mechanisms on the streamlines of the flow is shown. 
Figure 1.1 shows a schematic of the surface plasma actuator configuration used in the present work. The actuator consists of metal electrodes flushmounted on a dielectric plate, with one electrode powered (typically the cathode) and the other electrode grounded. Planar electrodes (two-dimensional) or pin electrodes (three-dimensional) may be used. At low discharge powers $(\sim 10-50 \mathrm{~W})$, the discharge exists in the glow regime with a luminous region visible above the cathode. The various mechanisms by which the surface direct-current discharge can affect a flow are also illustrated in Fig. 1.1. Electrostatic forcing, shown in Fig. 1.1(a), occurs in non-neutral regions of the surface plasma discharge which corresponds to the cathode sheath region in the case of direct-current discharges. Since the electrostatic force is towards the surface, the effect of electrostatic forcing is to push the flow streamlines towards the wall.

Volumetric and surface heating, shown in Fig. 1.1(b), occurs mainly in the vicinity of the cathode and leads to thickening of the boundary layer of the incoming flow due to dilatational effects. In the case of supersonic flow, the effect of volumetric heating is seen as a local surface pressure increase [8] accompanied by a weak shock wave at the cathode. The plasma actuator therefore behaves like a control surface. As mentioned above, one advantage of using a surface plasma discharge as a flow actuator is the high bandwidth: the surface plasma can be turned on and off in sub-millisecond time scales. The disadvantage of direct-current surface glow discharge plasmas as actuators is the relatively weak actuation forces due to low ionization fractions $\left(\sim 10^{-6}-10^{-4}\right)$. However, the direct-current surface glow discharge is one 
of the most basic actuator configurations and the objective of this study is to gain an understanding of the underlying physics of surface direct-current glow discharges in the presence of supersonic flow that could aid future surface plasma actuator designs.

In the presence of external magnetic fields, an additional body force due to Lorentz forcing $(\vec{j} \times \overrightarrow{\mathbf{B}})$ as seen in Fig. 1.1(c) can also affect the incoming flow. However, large current densities and external magnetic fields are required to ensure strong directional forces. The effect of external magnetic fields is not considered in this work.

The computational simulation of a surface plasma discharge interacting with a compressible supersonic flow is a highly stiff, multi-scale problem. The principal source of spatial stiffness results from the disparate characteristic dimensions associated with the localized surface discharge and the flow. For instance, the length scale associated with the flow could be $\sim 10$ 's $\mathrm{cm}$, whereas the typical surface plasma actuator device length is $\sim 1 \mathrm{~cm}$, and the plasma discharge is confined to a region of a few $\mathrm{mm}$ above the surface. Hence, for the simulation of a realistic flowfield with embedded regions of surface plasma actuators, the compressible flow equations could be solved on a larger domain appropriate to the overall flow length scales, with the plasma equations solved only on a smaller localized subdomain that is consistent with the length scale of the surface discharge. 


\subsection{Literature review}

In this section, we present a brief review of experimental work related to surface-based approaches to high speed flow control and continuum models of plasma discharges relevant to the current study.

\subsubsection{Surface-based approaches to high speed flow control}

Leonov et al. [9] investigated flow actuation at pressures 50-300 Torr with freestream Mach numbers 0.5-1.2 using plasma arcs. The typical electrical parameters in the experiment were power $\sim 1-10 \mathrm{~kW}$, and currents of few amperes. Gas temperatures in the discharge region were of order 1500-3000 K with vibrational temperatures of 5000-7000 K. The effect of the plasma was seen in the form of a shock wave, and a transverse magnetic field was found to effectively control the position of the shock. In subsequent work [10] the authors report that a transverse discharge with circular electrodes placed transversely with respect to the flow direction is more effective in depositing energy to the gas compared to the longitudinal configuration. They presented experimental results for Mach numbers 1.8-2 from various configurations including the discharge on a plane surface and on a backward facing step. Numerical simulations using the SST-turbulence model and five $1 \mathrm{~kW}$ heat sources were also shown, and the effect of the plasma was to reduce the pressure losses behind the step.

Kimmel et al. $[8,11]$ studied surface glow discharge effects on a laminar boundary layer at Mach 5. They used plane centimeter-scale electrodes such that the discharge was aligned with the flow direction (longitudinal config- 
uration). Peak electron densities of order $10^{12} \mathrm{~cm}^{-3}$ were measured using a Langmuir probe. For a $60 \mathrm{~W}$ discharge, the pressure increase observed was $\sim 10 \%$, and the pressure rise scaled linearly with power up to input powers of about 140 W. In Ref. [11], the authors reported that depending on the input power, either a diffuse luminous discharge above the cathode or a constricted discharge between the two electrodes were observed. The pressure rise measured just downstream of the cathode in the diffuse case $(140 \mathrm{~W})$ was found to be higher than the pressure rise in the constricted case $(170 \mathrm{~W})$. The structure of the discharge was altered in the presence of magnetic fields, but the authors were not able to conclusively determine whether the altered actuation was due to Lorentz forcing or due to the altered heating pattern. Computational simulations corresponding to the experiments have also been performed by Shang et al. $[12,13]$.

Shin et al. [14] performed a detailed characterization of pin-electrode direct-current plasma discharges in the presence of Mach 3 supersonic flow. At a given pressure, three discharge modes were observed: a diffuse mode at low currents $(\sim 10-100 \mathrm{~mA})$, a constricted mode at relatively higher current $(\sim 200$ $\mathrm{mA}$ ), and a bistable mode at intermediate currents where the discharge alternated between the diffuse and constricted modes. The discharge modes were observed both in the absence and presence of supersonic flow. The relatively low-power diffuse mode was found to produce a weak shockwave, but no shock was observed in the case of the high-power constricted mode. Peak rotational temperatures were estimated to be around 1200-1800 K in the absence of flow, and 500-800 $\mathrm{K}$ in the presence of supersonic flow. Different actuation char- 
acteristics were also observed by varying the relative position of the cathode: with the cathode upstream with respect to the flow, a weak shock was recorded in the diffuse mode but at the same power level a much weaker actuation was observed with the cathode downstream. The difference in actuation effects between cathode upstream and downstream was also observed by Menier et al. [15], who performed experiments of Mach 2 flow past a flat plate with plane electrodes.

\subsubsection{Continuum models of plasma discharges}

Continuum or fluid-based models of plasma discharges [16, 17, 18] are based on moments of the Boltzmann equation [19, 1], coupled to Poisson's equation to obtain the self-consistent electric field (in the absence of magnetic fields). Large spatial gradients of electron number densities in the sheath and disparities in characteristic timescales of the various plasma components can lead to numerical stiffness. The effect of boundary conditions on the stiffness of fluid models was studied by Wilcoxson and Manousiouthakis [20].

Qualitative and quantitative comparisons of plasma fluid model results with experimental measurements have been performed by several researchers. Boeuf and Pitchford [21] simulated a capacitively coupled RF discharge in argon at 100 mTorr and compared the plasma density results with experiments by Overzet et al. [22]. The simulations were able to qualitatively and quantitatively match the experimental density profiles and locations of the peak charge density. Bukowski et al. [23] compared their computed results from inductively coupled plasmas in chlorine and argon at pressures of 10 mTorr 
with experimental electron density, plasma potential and electron temperature measurements. While the computations displayed good qualitative agreement with the experimental data, the authors reported only a semi-quantitative agreement of their computed results. They further stated that the principal model assumption that lead to the quantitative discrepancy was the assumption of Maxwellian energy distribution function for electrons, but defended their choice by highlighting the complexity and relative uncertainties in alternative electron kinetics models. The issue of qualitative and quantitative agreement between experiments and plasma models was also discussed by Bogaerts and Gijbels [24], who presented three-dimensional simulations using a combination of fluid and Monte-Carlo approaches. Satisfactory agreement with comparisons of V-I characteristics, species densities and ion fluxes to the electrodes was reported. They comment on the uncertainties associated with input parameters in the computational models such as cross-section data that lead to uncertainties in the computed results, and also point out sources of uncertainty in experimental measurement techniques using Langmuir probes and laser-induced fluorescence spectroscopy.

One of the most comprehensive compilations of an air plasma chemistry is given by Kossyi et al. [25]. Their mechanism comprises $\sim 450$ reactions to represent finite rate kinetics in air, with ground state and electronically excited neutral species, and positive as well as negative ions. Vibrationally excited diatomic/polyatomic species were not included in their mechanism. Nahorny et al. [26] present a model with $\sim 430$ reactions similar to [25], with an added description of the vibrational kinetics of $\mathrm{O}_{2}, \mathrm{~N}_{2}$ and $\mathrm{NO}$. They used 
the model to perform a zero-dimensional (global) analysis of $\mathrm{N}_{2}-\mathrm{O}_{2}$ discharges at 2 Torr. The role of excited neutral atoms and molecules in $\mathrm{N}_{2}-\mathrm{O}_{2}$ discharges was subsequently studied by Gordiets et al. [27], who simplified the chemistry presented in Ref. [26] to include $\sim 120$ reactions. Castillo et al. [28] performed a series of experiments on direct-current hollow cathode discharges in the pressure range 2-35 mTorr, and developed a zero-dimensional model with $\sim 60$ reactions to help explain the experimental results. They found that $\mathrm{N}_{2}^{+}$and $\mathrm{NO}^{+}$were the dominant ion species over the investigated pressure range, and reasonable agreement with the experimental data was observed. Pancheshnyi et al. [29, 30] developed a reduced air chemistry model with 10 species and 15 reactions from based on the mechanism of Kossyi et al. [25] to simulate two-dimensional streamer discharges at pressures 300-760 Torr. Singh and Roy [31] used a simplified 8 species and 8 reactions chemistry model to compute dielectric barrier discharge (DBD) actuators at atmospheric pressure for low-speed flow control.

Computational models of direct-current glow discharges used to study high-speed flow control applications have been developed $[18,32,33]$ that use the drift-diffusion approximation to determine the species fluxes. In all of these models, simplified chemistries consisting of three species (positive ions, neutrals and electrons) are used along with the local field approximation (LFA) to determine electron and ion transport properties and rate coefficients. Deconinck et al. [34] studied supersonic flow effects on a surface plasma discharge in nitrogen by imposing a fixed flowfield velocity profile.

Parallel computing has become an indispensable tool used in several 
areas of computational research for large-scale simulations. To our knowledge, very few examples of parallelized plasma fluid models using the governing equations described in this section have been published in the literature. Young and $\mathrm{Wu}[35]$ developed a parallel vectorized code for computing rf discharges on a Cray X-MP, and a speedup of 1.988 was recorded on two processors. Hung et al. [36] presented a plasma fluid model parallelized using PETSc [37], and verified the model by simulating a quasi-1D argon rf discharge on 2-28 processors. Likhanskii et al. [38] have developed a parallel code for DBD actuators that they ran on up to 32 processors.

\subsection{Objectives and methodology}

The primary objective of this work is to obtain physical insight into the discharge structure, chemical composition, and energy deposition pathways of a surface plasma in the presence of a supersonic air flow using computational models to gain a better understanding of high-speed plasma actuators.

To model multi-dimensional glow discharge phenomena in air under typical actuator operating conditions, there is need for establishing a plasma chemical reaction mechanism that is capable of representing all important finite-rate chemistry effects in non-equilibrium air plasma. However, the plasma chemistry must be sufficiently reduced to enable feasible multidimensional computational simulations. Hence, we aim to develop and validate a plasma chemistry model that meets the following requirements: 1) To simulate nonequilibrium $\left(\mathrm{T}_{e} \sim 1-5 \mathrm{eV}, \mathrm{T}_{g} \sim 300-1500 \mathrm{~K}\right)$ glow discharge plasmas in air at pressures $\sim 1$ Torr (typical high-speed plasma actuator operating conditions); 
2) Sufficiently reduced for multidimensional plasma simulations and 3) can qualitatively and quantitatively reproduce experimental data for air glow discharges for high-speed plasma actuator operating conditions.

The air plasma model is self-consistently coupled to a compressible Navier-Stokes solver to study the effects of the surface plasma on supersonic flow and the effect of the flow on the discharge structure. The typical surface plasma actuator device length is $\sim 1 \mathrm{~cm}$, and the plasma discharge is confined to a region of a few mm above the surface. However, the length scale associated with the flow could be $\sim 10$ 's $\mathrm{cm}$, and solving the plasma model on the entire domain is neither feasible nor required for localized surface discharges in the present study. Hence, we aim to develop a combined model of the supersonic flow and the plasma with the ability to solve the plasma model on localized subdomains that are a subset of the overall simulation domain.

On the localized subdomains, both plasma and Navier-Stokes models are solved using an iteratively decoupled algorithm. Source terms are exchanged between the two models in each combined iteration: the bulk flow field pressure, velocity and gas temperature from the Navier-Stokes solver are used as inputs to the plasma model, and electrostatic force (momentum source) and volumetric heating (energy source) terms from the plasma model are used as inputs to the Navier-Stokes solver.

While the strategy outlined above allows us to study plane plasma actuators (2-D) in the presence of supersonic flow, there are practical limitations on the total mesh size and the number of species/reactions that can be 
included in the plasma chemistry model. Moreover, the actuators in practice have three dimensional geometry, for instance with circular pin-like electrodes. Hence three dimensional simulations of plasma discharges are necessary, and are impractical to do using a serial (single-processor) model implementation. The coupled plasma-compressible flow model is therefore parallelized using domain decomposition techniques (MPI), that enables the investigation of 3-D plasma actuators such as pin electrode actuators used in experiments [14].

\subsection{Contributions of the present research}

The principal contributions of the present work are:

- Development and validation of an air plasma chemistry model for pressures $\sim 1-20$ Torr.

- Implementation of a self-consistent coupled plasma discharge-compressible flow model on realistic geometries.

- Investigation of the discharge structure, chemical composition and energy deposition pathways of an air plasma discharge in supersonic flow.

- Domain decomposition based parallelization of plasma and supersonic flow models to study the physics of three-dimensional pin-electrode surface actuators. 


\section{Chapter 2}

\section{Model Description}

In this chapter, the fluid-based plasma model and compressible NavierStokes solver are described. The plasma model has been developed as a group effort [39, 40, 41, 42, 43] since 2007, while the Navier-Stokes code has been implemented primarily by the author to examine the physics of the plasma discharge in the presence of supersonic flow. In addition, each governing equation can be solved on select physical subdomains that are a subset of the overall problem domain. Both solver codes are implemented in $\mathrm{C}++$ with the same underlying mesh infrastructure and can be solved either independently in standalone fashion or in combination with each other. The models are discussed here in detail with emphasis on aspects most relevant to the present work.

\subsection{Plasma Model}

\subsubsection{Governing Equations}

The glow discharge model is based on a self-consistent multi-species continuum description of a plasma with finite-rate chemistry. Species continuity equations

$$
\frac{\partial n_{k}}{\partial t}+\vec{\nabla} \cdot \vec{f}_{k}=\dot{G}_{k} \quad\left(k=1, . ., K_{g}, k \neq k_{b}\right)
$$


are solved for each species $k$ in the discharge, except for a single "dominant background species" $k_{b}$ ( $\mathrm{N}_{2}$ in the case of an air plasma). In (2.1), $n_{k}$ is the species number density, $\vec{f}_{k}$ is the species number flux and $\dot{G}_{k}$ is the net rate of production of species $k$ due to chemical reactions. The dominant background species density is determined using the ideal gas law

$$
p=\sum_{k}^{K_{g}} n_{k} k_{B} T_{k}
$$

In (2.2), the pressure is specified as a constant when the plasma model is solved standalone, and is obtained from the Navier-Stokes solver as an input when the two solvers are combined. The species number flux in(2.1) is approximated by

$$
\overrightarrow{f_{k}}=n_{k} \vec{u}_{k}=-\mu_{k} n_{k} \vec{\nabla} \phi-D_{k} \vec{\nabla} n_{k}+n_{k} \vec{V}
$$

Equation (2.3) is essentially the drift-diffusion approximation with an additional bulk convection term, where $\vec{V}$ denotes the convective velocity. The drift-diffusion approximation is a simplification of the species momentum equation for a collisional plasma commonly used in glow discharge modeling [16, 18]. The potential $\phi$ in (2.3) is obtained self-consistently by solving Poisson's equation

$$
\nabla^{2} \phi=-\frac{e}{\epsilon_{0}} \sum_{k} Z_{k} n_{k}
$$

where $Z_{k}$ is the charge number of species $k, \mu_{k}$ is the species mobility and $D_{k}$ is the species diffusion coefficient. In most plasma actuator discharge modeling studies, the local field approximation (LFA) is used. In the LFA, electrons 
are assumed to be in equilibrium with the reduced electric field $\left(E / N_{g}\right)$ owing to relatively high pressure conditions for these discharges. However, the LFA may be inaccurate due to non-local transport effects at relatively low pressures and in the cathode fall region of the discharge where the electric field is strong [44]. We therefore solve the complete electron energy conservation equation to determine the mean electron energy $e_{e}$,

$$
\frac{\partial e_{e}}{\partial t}+\vec{\nabla} \cdot\left(\left(\frac{5}{3} \mu_{e} \vec{E}+\vec{V}\right) e_{e}-\frac{5}{3} D_{e} \vec{\nabla} e_{e}\right)=S_{e_{e}}
$$

where

$$
S_{e_{e}}=+e \overrightarrow{f_{e}} \cdot \vec{\nabla} \phi-e \sum_{i} \Delta E_{i}^{e} r_{i}-\frac{3}{2} k_{B} n_{e} \frac{2 m_{e}}{m_{k_{b}}}\left(T_{e}-T_{g}\right) \bar{\nu}_{e, k_{b}}
$$

In (2.5), $e_{e}=\frac{3}{2} n_{e} k_{B} T_{e}$ is the total mean electron energy per unit volume, $\mu_{e}$ is the electron mobility, $\vec{E}$ is the electric field $(\vec{E}=-\vec{\nabla} \phi), \vec{V}$ is the bulk flow velocity ( $=0$ in the case of a stationary plasma) and $D_{e}$ is the electron diffusion coefficient. On the right-hand side of (2.5) are the source terms denoted by $S_{e_{e}}$ : the electron Joule heating term $e \overrightarrow{f_{e}} \cdot \vec{\nabla} \phi$, the inelastic collision loss term $e \sum_{i} \Delta E_{i} r_{i}$, and the elastic collision loss term. $\Delta E_{i}^{e}$ is the energy lost for inelastic collision corresponding to reaction $i$, with rate of progress $r_{i}$. The electron transport coefficients are assumed to be functions of the local mean electron temperature obtained from the BOLSIG+ Boltzmann equation solver [45]. The heavy species (ions, neutrals) are assumed to have a common temperature $T_{g}$, which can be obtained by solving the gas energy equation. 
The gas energy conservation equation can be written as follows:

$$
\frac{\partial \sum_{k \in H} n_{k} h_{k}}{\partial t}+\vec{\nabla} \cdot\left(\sum_{k \in H} \overrightarrow{f_{k}} h_{k}-\sum_{k \in H} \kappa_{k} \vec{\nabla} T_{g}\right)=S_{T_{g}}
$$

where $H$ denotes the set of all heavy species and $h_{k}=\int_{0}^{T_{g}} C_{p_{k}} T_{k} \approx C_{p_{k}} T_{g}$ is the sensible enthalpy of species $\mathrm{k}$, and $S_{T_{g}}$ denotes the gas energy source terms, given by

$$
S_{T_{g}}=\eta_{\mathrm{Th}}\left(-e \sum_{k \in H} Z_{k} \vec{f}_{k} \cdot \vec{\nabla} \phi\right)+\frac{3}{2} k_{B} n_{e} \frac{2 m_{e}}{m_{k_{b}}}\left(T_{e}-T_{g}\right) \bar{\nu}_{e, k_{b}}-e \sum_{i} \Delta E_{i}^{g} r_{i}
$$

The gas energy source term in (2.7) has three components. The first source of gas heating in (2.7) is the ion Joule heating term. In plasma discharges, if the ion mean free path is comparable to the cathode sheath thickness, the ions gain kinetic energy from the electric field but fail to thermalize with the neutrals before they hit the surface of the electrode. As the current density increases, the sheath thickness decreases and the fraction of energetic ions that transfer kinetic energy to the gas decreases. Revel et al. [46] compared the ion Joule heating term calculated by a fluid model in an argon DC discharge with a more accurate Monte-Carlo simulation of ion transport. They found that the gas heating in the cathode sheath was non-local and the fluid model overpredicted the ion Joule heating in the sheath. Hence, to account for the fact that only a fraction of the computed ion Joule heating term is actually transferred to the gas energy pool, we include an empirical factor $\eta_{\mathrm{Th}}$ which represents the fraction of ion kinetic energy that is thermalized with the neutral gas [47]. The energy fraction $\left(1-\eta_{\mathrm{Th}}\right)$ of the ion Joule heating term is assumed to be lost to the surface. 
The second source term in (2.7) is the energy gained by the neutral gas pool due to elastic collisions with electrons. The final source term in (2.7) is also an inelastic collision source term, due to energy loss/gain from reactions involving ions/excited species and neutrals, such as non-resonant charge transfer reactions. The inelastic collision source term also includes energy transferred from electrons to rotational, vibrational and electronic excited states of the neutral gas molecules $\left(\mathrm{N}_{2}\right.$ and $\mathrm{O}_{2}$, in the case of air) which then thermalize with the neutral gas. For a stationary discharge, a reasonable assumption is that at steady state, all the energy deposited into excited states of the neutral gas is eventually transferred to the neutral gas energy pool. For a plasma discharge in the presence of a flow, the lifetime of the metastable and vibrationally excited states compared to the flow timescales determines the fraction of energy that is transferred to the gas in the discharge region. This issue will be discussed in further detail in the sections on plasma chemistry and the results.

In the case where the plasma discharge model is solved in conjunction with the Navier-Stokes flow model, the gas energy equation with source terms given in (2.7) is solved as a part of the flow model and the plasma gas energy equation (2.6) is not solved.

\subsubsection{Boundary conditions}

Boundary conditions for the governing equations discussed in the previous section are as follows: 


\subsubsection{Species continuity boundary conditions}

For solid surfaces, assuming that electrons are in equilibrium at temperature $T_{e}$ near the wall, the net number flux of electrons from the discharge towards the wall can be written as

$$
\vec{f}_{e} \cdot \hat{n}_{s}=\frac{1}{4} n_{e}\left(\frac{8 k_{B} T_{e}}{\pi m_{e}}\right)^{1 / 2}-\sum_{k} \gamma_{k} f_{k}^{s+}
$$

where $\gamma_{k}$ is the secondary electron emission coefficient of species $k$, and $f_{k}^{s+}$ is the flux of all species $k$ directed towards the wall. $\hat{n}_{s}$ is the outward unit vector normal to the surface. For ions,

$$
\overrightarrow{f_{i}} \cdot \hat{n}_{s}=f_{i}^{s+}=\frac{1}{4} n_{i}\left(\frac{8 k_{B} T_{g}}{\pi m_{i}}\right)^{1 / 2}+n_{i} \max \left(0,-\mu_{i} \hat{n}_{s} \cdot \vec{\nabla} \phi\right)
$$

and for neutrals,

$$
\vec{f}_{n} \cdot \hat{n}_{s}=f_{n}^{s+}=\frac{1}{4} n_{n}\left(\frac{8 k_{B} T_{g}}{\pi m_{n}}\right)^{1 / 2}
$$

In (2.9) and (2.10) we have made the implicit assumption that all heavy species have the same gas temperature $T_{g}$. The second term in (2.9) represents the mobility-limited flux for ions. In the case of positive ions, if the electric field is directed towards the wall, the mobility-limited flux term is positive, i.e. positive ions drift towards the wall. For negative ions, an electric field directed towards the surface keeps negative ions from drifting to the wall, resulting in a zero negative ion drift flux to the wall. The plasma model includes the capability of modeling surface reactions, and so the sign of the net flux of ions and neutrals to the wall depends on the surface chemistry. For the glow discharges studied in this work, neutral species are produced by surface recombination of ions and hence the net flux of neutrals towards a solid surface is negative. 
For symmetry boundaries, the fluxes of all species $k$ normal to the boundary are set to zero, i.e.

$$
\vec{f}_{k} \cdot \hat{n}_{s}=0
$$

An outflow boundary condition is defined such that the species are convected out of the domain with flux

$$
\vec{f}_{k} \cdot \hat{n}_{s}=n_{k} \vec{V}
$$

where $\vec{V}$ is the convective velocity defined in (2.3), and the number density $n_{k}$ is extrapolated from the interior. In addition, Dirichlet boundary conditions can be imposed in the form of fixed species densities and fixed species mole fractions.

\subsubsection{Potential boundary conditions}

At electrode surfaces, the potential is usually specified as a constant or calculated implicitly if an external circuit is present. In either case, the potential boundary condition at electrodes is essentially a Dirichlet boundary condition. At dielectric surfaces, charged species trapping can occur resulting in a local surface charge induced electric field. To model this phenomenon, we solve an ODE to determine the surface charge density $\rho_{s}$

$$
\frac{\partial \rho_{s}}{\partial t}=e \sum_{k} Z_{k} \vec{f}_{k} \cdot \hat{n}_{s}
$$

Once $\rho_{s}$ is computed from equation (2.13), the potential at a thin dielectric boundary surface can be found analytically from Gauss' Law using the inter-

face condition $\left(\vec{D}_{\text {dielectric }}-\vec{D}_{\text {interior }}\right) \cdot \hat{n}_{s}=\rho_{s}$, where $\vec{D}=\epsilon \vec{E}$ is the electric flux 
density. For a thin dielectric, the potential on the boundary can be written as

$$
\phi_{s}=\frac{\phi_{c}+\frac{\delta}{\epsilon_{0}}\left(\rho_{s}+\frac{\epsilon_{d}}{d} \phi_{\text {back }}\right)}{1+\frac{\delta}{\epsilon_{0}} \frac{\epsilon_{d}}{d}}
$$

In $(2.14), \phi_{c}$ is the potential in the solution domain (interior) at a distance $\delta$ from the boundary, $\epsilon_{d}$ and $d$ are the permittivity and thickness of the dielectric respectively, and $\phi_{\text {back }}$ is the potential on the other side of the dielectric. At symmetry boundaries, the normal component of the potential gradient is set to zero:

$$
\vec{\nabla} \phi \cdot \hat{n}_{s}=0
$$

\subsubsection{Electron and gas energy boundary conditions}

The energy flux of electrons towards a solid surface is given by

$$
\overrightarrow{Q_{e}} \cdot \hat{n}_{s}=\varepsilon_{s e}\left(\frac{1}{4} n_{e}\left(\frac{8 k_{B} T_{e}}{\pi m_{e}}\right)^{1 / 2}\right)-e \Delta E_{s e}^{e} \sum_{k} \gamma_{k} f_{k}^{s+}
$$

Equation (2.16) follows from (2.8), where every electron that hits the surface is assumed to carry $\varepsilon_{s e}$ units of energy. For the simulations presented in this work, $\varepsilon_{s e}$ was assumed to have a value $\frac{5}{2} k_{B} T_{e}$. A more appropriate choice for $\varepsilon_{s e}$ is $2 k_{B} T_{e}$, which can be derived from a Maxwellian distribution function for electrons, and is recommended for future work. For discharges studied in the present work, with all other simulation parameters being exactly the same, the difference between results corresponding to $\varepsilon_{s e}=\frac{5}{2} k_{B} T_{e}$ and $\varepsilon_{s e}=2 k_{B} T_{e}$ are negligibly small.

$\Delta E_{s e}^{e}$ is the energy with which secondary electrons enter the solution domain, which depends on material properties such as the work function of the 
surface. As in the case of the species continuity equation, symmetry condition is imposed by setting the normal flux to zero, i.e.

$$
\overrightarrow{Q_{e}} \cdot \hat{n}_{s}=0
$$

For the gas energy equation (2.6), Dirichlet boundary conditions are applied at solid surfaces and a symmetry boundary condition is imposed similar to $(2.17)$.

\subsubsection{Spatial and temporal discretization}

The species density, potential, electron energy and gas energy equations described above can be cast as transient convection diffusion-source conservation equations that are written in the integral form as

$$
\iiint_{V} \frac{\partial \varphi}{\partial t} d V+\iint_{\partial V}\left(\vec{c}_{\varphi} \varphi-D_{\varphi} \vec{\nabla} \varphi\right) \cdot \hat{n} d S=\iiint_{V} S_{\varphi} d V
$$

Here, $\varphi$ is the dependent variable (e.g., species density $n_{k}$ or electron energy $\left.e_{e}\right), \vec{c}_{\varphi}$ is the convective velocity, $D_{\varphi}$ is the diffusion coefficient associated with the transport of $\varphi$ and $S_{\varphi}$ is the volumetric source term that represents the net production of $\varphi$ in a control volume $V$ bounded by closed control surface $\partial V$. The equations are discretized using a cell-centered finite-

volume scheme in a generalized mesh framework. Writing (2.18) in discrete form for a cell $c$

$$
\left(\frac{\partial \varphi}{\partial t}\right)_{c} V_{c}+\sum_{f=1}^{N_{f}}\left(\vec{c}_{\varphi} \varphi-D_{\varphi} \vec{\nabla} \varphi\right)_{f} \cdot \vec{A}_{f}=\left(S_{\varphi}\right)_{c} V_{c}
$$


Spatial discretization of the fluxes in (2.19) is performed using the firstorder upwind scheme or the Scharfetter-Gummel exponential scheme [48] using the formulation of Patankar [49]. The transport coefficients in (2.19) are typically "lagged", i.e. calculated using values of properties at the previous timestep.

On structured mesh implementations, the Joule heating source terms are typically evaluated using multi-dimensional averaging [50]. On unstructured meshes, multi-dimensional averaging can lead to erroneous results since the faces of the grid cells are not aligned with the Cartesian coordinate directions. A species flux reconstruction approach [41] for unstructured grids is used to evaluate the Joule heating source terms appearing in the electron energy equation (2.5) and in the gas energy equation (2.6).

The potential equation (2.4) is a specialized form of (2.18) with the time derivative and convection terms set to zero, and diffusion coefficient $D_{\phi}=\epsilon_{r}$, where $\epsilon_{r}$ is the relative permittivity of the medium. In practice however, a purely explicit discretization of the potential equation in a plasma discharge leads to very stringent restrictions on the time step used in the electron species continuity and electron energy equations. The stiffness arises from the coupling between the potential equation, which has the net species number density $\sum Z_{k} n_{k}$ in the source term, and the species continuity fluxes $\vec{f}_{k}$, which have the electric field appearing in the drift term in (2.3). In the bulk plasma, the net species density is not identically zero but a very small value associated with the ambipolar electric field that maintains quasi-neutrality in the bulk plasma. Small perturbations in the species densities can lead to an unstable numerical 
solution of the species continuity-Poisson system. The characteristic timescale for coupling between the charged species density and potential equations is the dielectric relaxation timestep $\Delta t_{d}=\epsilon_{0} / \sigma$, where $\sigma$ is the plasma conductivity, which can be smaller than the limiting CFL timestep $\frac{\Delta x}{\mu_{e} E}$. To overcome the limitation of the dielectric relaxation timestep, the source term in the potential equation is written as

$$
-\vec{\nabla} \cdot\left[\epsilon_{r} \vec{\nabla} \phi^{m+1}\right]=\frac{e}{\epsilon_{0}}\left(\sum_{i} Z_{i} n_{i}^{m}-n_{e}^{m+1}\right)
$$

In (2.20), the solution is sought for time level $(m+1)$ and $m$ denotes the current time level. The ions in (2.20) are treated explicitly since the ion time scales are typically much larger than the electron time scale. The electron density $n_{e}^{m+1}$ is estimated using the electron continuity equation

$$
n_{e}^{m+1}=n_{e}^{m}-\Delta t \vec{\nabla} \cdot\left[\left(n_{e} \vec{V}-D_{e} \vec{\nabla} n_{e}\right)^{m}-\left(\mu_{e} n_{e}\right)^{m} \vec{\nabla} \phi^{m+1}\right]+\Delta t \dot{G}_{e}^{m}
$$

In the above equation, the potential gradient term is treated implicitly (at time level $m+1$ ). Substituting for $n_{e}^{m+1}$ in (2.20), and grouping all terms at time level $m+1$ on the left hand side, we get

$$
\begin{aligned}
-\vec{\nabla} \cdot\left[\left(\epsilon_{r}-\frac{e}{\epsilon_{0}} \mu_{e}^{m} n_{e}^{m} \Delta t\right) \vec{\nabla} \phi^{m+1}\right]= & \frac{e}{\epsilon_{0}}\left(\sum_{i} Z_{i} n_{i}^{m}-n_{e}^{m}\right) \\
& +\frac{e}{\epsilon_{0}} \Delta t\left(\vec{\nabla} \cdot \vec{f}_{e}^{m}-\dot{G}_{e}^{m}\right)
\end{aligned}
$$

In (2.22), $\vec{f}_{e}^{\prime}$ is the electron flux from (2.3) without the mobility flux contribution. The electron mobility flux has been taken to the left hand side 
so the effective diffusion coefficient is $D_{\phi}=\epsilon_{r}-\frac{e}{\epsilon_{0}} \mu_{e}^{m} n_{e}^{m} \Delta t$. By solving (2.22), we can take time steps that are $\sim 10$ times larger than the dielectric relaxation timestep without loss of accuracy. Similar approaches have been taken by Boeuf et al. [21], and Hagelaar et al. [51].

The discretized equations are non-dimensionalized and solved using Krylov subspace (KSP) iterative methods from PETSc [37]. A time-splitting approach is used to solve different governing equations. This approach entails solving each governing equation individually in a segregated fashion with characteristic time steps defined by accuracy and stability criterion for that equation, as shown in Fig. 2.1.

The electron species conservation, electron energy and Poisson's equation are solved at every time step with the smallest time step size (typically $\sim 10^{-12}-10^{-10} \mathrm{~s}$ for the simulations presented in this work). Since we seek a steady state solution, a larger time step of the order of $10^{-9}-10^{-8} \mathrm{~s}$ may be used for neutral species. For certain problems, it is also possible to take time steps larger than the electron time step for positive and negative ions. However, in the present study, the positive ion time step was set to equal the electron time step. In addition, at a given electron time step, the species conservation equations for the heavy species can be skipped, i.e. the neutral species conservation equations can be solved once for every 10 electron time steps, for example. The gas chemistry source terms can also be frozen at a given electron time step and updated once every 2 to 5 electron time steps. Likewise, the heavy species transport properties, which vary on relatively larger time scales, are updated less frequently (every 10-100 electron 


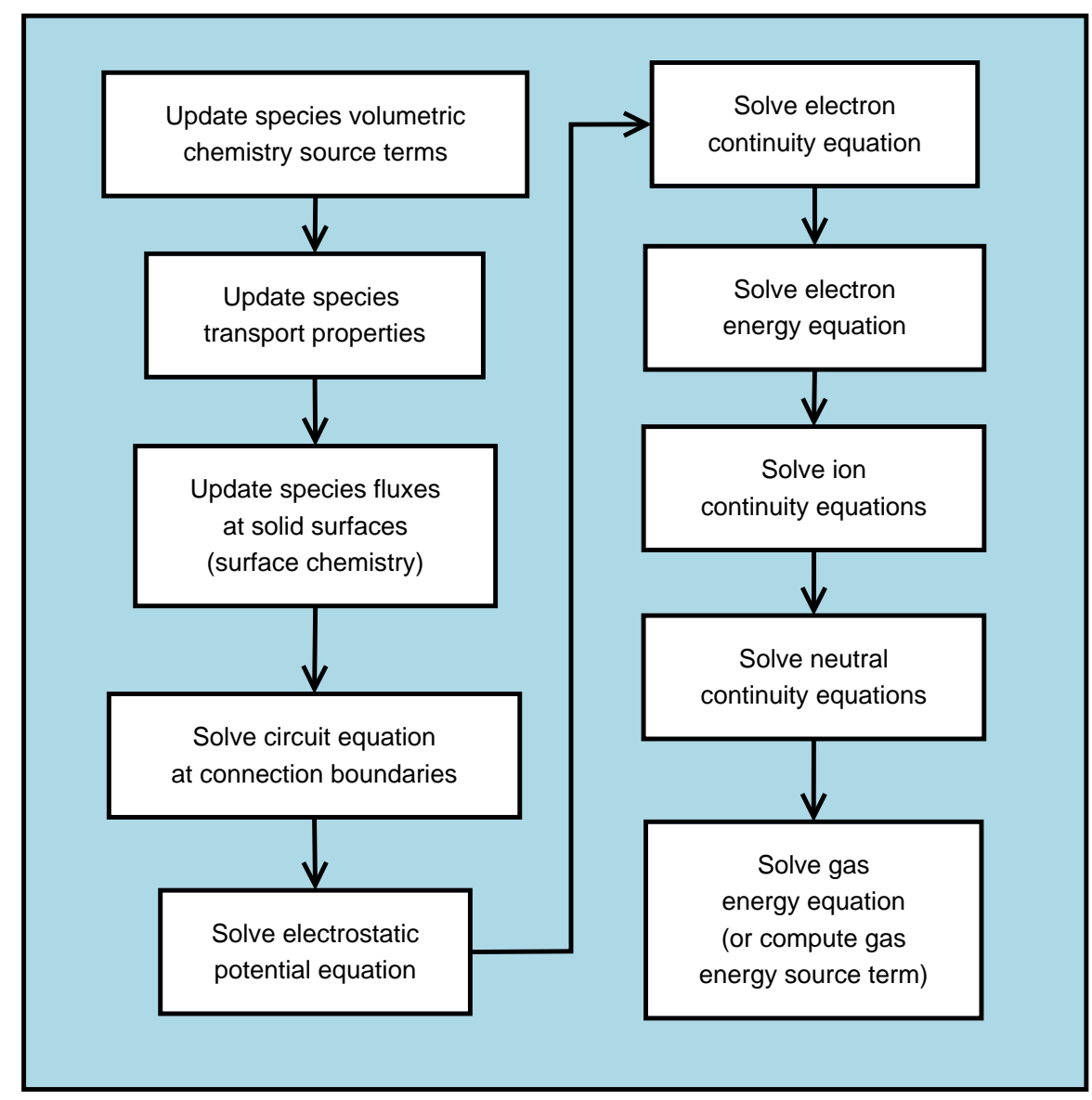

Figure 2.1: Equations solved in the plasma model in a given time step.

time steps). The strategy described above can lead to significant reduction in the overall computational time to reach a steady state since it allows individual governing equations to evolve according to their own characteristic physical timescales resulting in significant alleviation of the temporal stiffness problem in the model. In addition, non-dimensionalization of the discretized equations results in better conditioned matrices that improve the convergence rate of the iterative KSP algorithm in each timestep. 


\subsection{Navier-Stokes Solver}

\subsubsection{Governing equations and boundary conditions}

A cell-centered finite volume compressible Navier-Stokes solver has been implemented on the same generalized grid framework as the plasma model described above and can solve 2-D, axisymmetric and $3-\mathrm{D}$ problems. In two dimensions, the compressible Navier-Stokes flow equations are written as

$$
\frac{d \mathbf{U}}{d t}+\vec{\nabla} \cdot \overrightarrow{\mathbf{F}}_{\text {inviscid }}=\vec{\nabla} \cdot \overrightarrow{\mathbf{F}}_{\text {visc }}+\mathbf{S}
$$

where,

$$
\begin{aligned}
& \mathbf{U}=\left[\begin{array}{c}
\rho \\
\rho u \\
\rho v \\
\rho e_{t}
\end{array}\right] \\
& \overrightarrow{\mathbf{F}}_{\text {inviscid }}=\left[\begin{array}{c}
\rho u \\
\rho u^{2}+p \\
\rho u v \\
\left(\rho e_{t}+p\right) u
\end{array}\right] \hat{\mathbf{i}}+\left[\begin{array}{c}
\rho v \\
\rho v u \\
\rho v^{2}+p \\
\left(\rho e_{t}+p\right) v
\end{array}\right] \hat{\mathbf{j}} \\
& \overrightarrow{\mathbf{F}}_{\text {viscous }}=\left[\begin{array}{c}
0 \\
\tau_{x x} \\
\tau_{x y} \\
u \tau_{x x}+v \tau_{x y}+-\dot{q}_{x}
\end{array}\right] \hat{\mathbf{i}}+\left[\begin{array}{c}
0 \\
\tau_{y x} \\
\tau_{y y} \\
u \tau_{y x}+v \tau_{y y}-\dot{q}_{y}
\end{array}\right] \hat{\mathbf{j}} \\
& \mathbf{S}=\left[\begin{array}{c}
0 \\
f_{x} \\
f_{y} \\
\vec{f} \cdot \vec{V}+S_{T_{g}}
\end{array}\right]
\end{aligned}
$$

Primitive variables of the governing equations include the fluid velocity $\vec{V}=u \hat{i}+v \hat{j}$, the density $\rho$ of a single composite neutral species (air), pressure 
$p$ and the total fluid energy $e_{t}=\frac{p}{(\gamma-1) \rho}+\frac{1}{2} V^{2}$ (thermal + kinetic), where $\gamma$ is the ratio of specific heats for the fluid. U represents the conservative variables, $\mathbf{F}_{\text {inviscid }}$ and $\mathbf{F}_{\text {viscous }}$ represent the inviscid and viscous flux vectors, respectively, and $\mathbf{S}$ represents the vector of source terms. Closure of the governing equations is achieved using the Fourier law $\dot{q}_{x}=-k \frac{\partial T}{\partial x}, \dot{q}_{y}=-k \frac{\partial T}{\partial y}$ for the heat flux components and $\tau_{x x}=\frac{2 \mu}{3}\left(2 \frac{\partial u}{\partial x}-\frac{\partial u}{\partial y}\right), \tau_{y y}=\frac{2 \mu}{3}\left(2 \frac{\partial v}{\partial y}-\frac{\partial u}{\partial x}\right), \tau_{x y}=\mu\left(\frac{\partial u}{\partial y}+\frac{\partial v}{\partial x}\right)$ for the viscous stress terms. The effect of plasma flow actuation appears in the body force (per unit volume) term $\vec{f}_{E S}=f_{x} \hat{i}+f_{y} \hat{j}$, and $S_{T_{g}}$ the volumetric heat source term given in (2.7). The viscosity $\mu$ is calculated using Sutherlands law [52] and the thermal conductivity is computed assuming a constant Prandtl number $\operatorname{Pr}=0.72$ for air. Characteristic-based boundary conditions are implemented for inflow and outflow boundaries while no-slip boundary conditions are implemented at solid wall surfaces. Constant temperature wall boundary conditions are also implemented for the energy equation at the wall surfaces.

\subsubsection{Spatial and temporal discretization}

The above flow governing equations are discretized in space based on the finite volume approach. Second-order spatial discretization is achieved using the Green-Gauss gradient reconstruction $[53,54]$ of the solution variables, with slope limiting of the solution variables using the Venkatakrishnan [55] scheme to preserve monotonicity of the reconstruction. The inviscid flux terms are discretized using the AUSM+ [56] upwind scheme. The viscous flux terms are discretized using the approach of Haselbacher and Blazek [57] to prevent 
the decoupling of solution on quadrilateral or hexahedral meshes. Temporal discretization is achieved using a second-order backward Euler scheme with a dual-time stepping approach for relaxation of the solution at each time step using a Runge-Kutta based algorithm [58] or using an implicit GMRES algorithm with ILU preconditioning $[59,60]$.

\subsection{Coupled plasma-compressible flow solution method- ology}

The problem of a surface plasma discharge interacting with a flow is a highly stiff, multi-scale problem. Temporal stiffness occurs due to very small time scales associated with plasma phenomena compared to flow time scales. Spatial stiffness also results from the disparate characteristic dimensions associated with the localized surface discharge and the flow. To simulate the plasma discharge in the supersonic air stream, the plasma solver described in Section 2.1 is coupled with the compressible Navier-Stokes solver described in Section 2.2 in a self-consistent manner. Source terms for the momentum and energy equations, $\vec{f}_{E S}$ and $S_{T_{g}}$ respectively are calculated from the plasma model and input into the Navier-Stokes solver. In turn, the pressure $p$, gas

temperature $T_{g}$ and velocity field $\vec{V}$ from the Navier-Stokes solution are fed back into the plasma model. The combined plasma-model is illustrated in Fig. 2.2.

At each update to the plasma solver, the densities of $\mathrm{N}_{2}$ and $\mathrm{O}_{2}$ are recalculated with fixed mole fractions 0.79 and 0.21 respectively based on the neutral gas density $\rho$ to ensure consistency with the Navier-Stokes solver. Both 


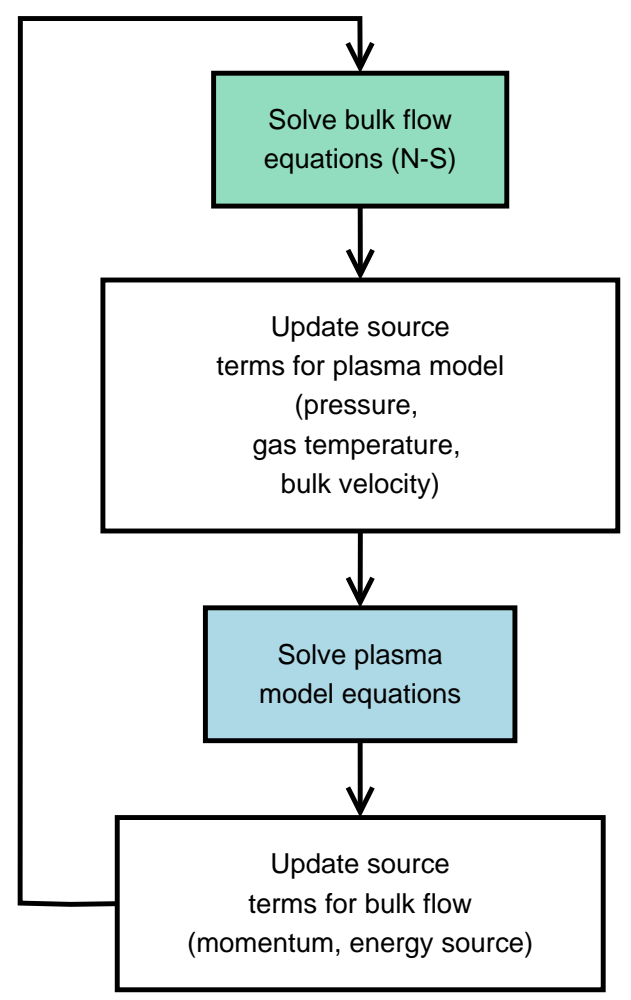

Figure 2.2: Flowchart of the combined plasma-flow model.

models are solved on the same computational mesh, i.e. no interpolation of source terms is performed, thereby ensuring conservation of energy and mass. The solutions are iterated back and forth between the two solvers till a steady state is reached. 


\section{Chapter 3}

\section{Air Plasma Discharge Validation}

Modeling multi-dimensional glow discharge phenomena in air requires a plasma chemical reaction mechanism that is capable of representing important finite-rate chemistry effects in the non-equilibrium air plasma while being sufficiently reduced to enable feasible multidimensional computational simulations. Hence, as a part of the current research, we aim to develop and validate a plasma chemistry model that can: $(1)$ simulate nonequilibrium $\left(T_{e} \sim 1-5 \mathrm{eV}\right.$, $\left.T_{g} \sim 300-1500 \mathrm{~K}\right)$ glow discharge plasmas in air at pressures $\sim 1$-20 Torr, and (2) is sufficiently reduced so we can use it in 2-D and 3-D multidimensional applications. In this chapter, the air plasma chemistry mechanism including the species and reactions considered, reaction rate coefficients and transport coefficients is described. Then, the air plasma model is validated against an experiment from the literature corresponding to typical high-speed actuator operating conditions.

\subsection{Air plasma chemistry and transport}

The reaction mechanism used in the present study is primarily adopted from Ref. [30], in which the authors compile a reduced mechanism of 10 species and 15 reactions derived from a more comprehensive mechanism in Ref. [25], 
which includes $\sim 450$ reactions. A list of the reactions and their rate coefficients are given in Table 3.1. The air plasma chemistry used in this study has 11 species: e, $\mathrm{N}_{2}, \mathrm{O}_{2}, \mathrm{~N}_{2}^{+}, \mathrm{N}_{4}^{+}, \mathrm{O}_{2}^{+}, \mathrm{O}_{4}^{+}, \mathrm{O}_{2}^{+} \mathrm{N}_{2}, \mathrm{O}_{2}^{-}, \mathrm{O}^{-}$and $\mathrm{O}$. The reaction rate coefficients for all electron impact reactions with a finite energy threshold are obtained using an off-line zero-dimensional electron Boltzmann solver BOLSIG+ [45].

\begin{tabular}{|c|c|c|c|c|c|}
\hline & Reaction & Rate $^{a}$ & $\Delta E^{e}(\mathrm{eV})$ & $\Delta E^{g}(\mathrm{eV})$ & Ref. \\
\hline $\mathrm{G}_{1}$ & $\mathrm{e}^{-}+\mathrm{N}_{2} \rightarrow 2 \mathrm{e}^{-}+\mathrm{N}_{2}^{+}$ & BOLSIG+ & 15.6 & & [45] \\
\hline $\mathrm{G}_{2}$ & $\mathrm{e}^{-}+\mathrm{O}_{2} \rightarrow 2 \mathrm{e}^{-}+\mathrm{O}_{2}^{+}$ & BOLSIG+ & 12.07 & & {$[45]$} \\
\hline $\mathrm{G}_{3}$ & $\mathrm{~N}_{2}^{+}+\mathrm{N}_{2}+\mathrm{M} \rightarrow \mathrm{N}_{4}^{+}+\mathrm{M}$ & $5 \times 10^{-41}$ & & & {$[25]^{b}$} \\
\hline $\mathrm{G}_{4}$ & $\mathrm{~N}_{4}^{+}+\mathrm{O}_{2} \rightarrow \mathrm{O}_{2}^{+}+2 \mathrm{~N}_{2}$ & $2.5 \times 10^{-16}$ & & -3.51 & {$[25]$} \\
\hline $\mathrm{G}_{5}$ & $\mathrm{~N}_{2}^{+}+\mathrm{O}_{2} \rightarrow \mathrm{O}_{2}^{+}+\mathrm{N}_{2}$ & $1.05 \times 10^{-15} T_{g}^{-0.5}$ & & -3.51 & {$[25]$} \\
\hline $\mathrm{G}_{6}$ & $\mathrm{O}_{2}^{+}+2 \mathrm{~N}_{2} \rightarrow \mathrm{O}_{2}^{+} \mathrm{N}_{2}+\mathrm{N}_{2}$ & $8.1 \times 10^{-38} T_{g}^{-2.0}$ & & & {$[25]$} \\
\hline $\mathrm{G}_{7}$ & $\mathrm{O}_{2}^{+} \mathrm{N}_{2}+\mathrm{N}_{2} \rightarrow \mathrm{O}_{2}^{+}+2 \mathrm{~N}_{2}$ & $14.8 T_{g}^{-5.3} e^{2357 / T_{g}}$ & & & {$[25]$} \\
\hline $\mathrm{G}_{8}$ & $\mathrm{O}_{2}^{+} \mathrm{N}_{2}+\mathrm{O}_{2} \rightarrow \mathrm{O}_{4}^{+}+\mathrm{N}_{2}$ & $1 \times 10^{-15}$ & & & {$[25]$} \\
\hline $\mathrm{G}_{9}$ & $\mathrm{O}_{2}^{+}+\mathrm{O}_{2}+\mathrm{M} \rightarrow \mathrm{O}_{4}^{+}+\mathrm{M}$ & $2.03 \times 10^{-34} T_{g}^{-3.2}$ & & & {$[25]^{b}$} \\
\hline $\mathrm{G}_{10}$ & $\mathrm{e}^{-}+\mathrm{O}_{4}^{+} \rightarrow 2 \mathrm{O}_{2}$ & $2.42 \times 10^{-11} T_{e}^{-0.5}$ & & -12.07 & {$[25]$} \\
\hline $\mathrm{G}_{11}$ & $\mathrm{e}^{-}+\mathrm{O}_{2}^{+} \rightarrow \mathrm{O}_{2}$ & $6 \times 10^{-11} T_{e}^{-1.0}$ & & -6.91 & {$[25]$} \\
\hline $\mathrm{G}_{12}$ & $\mathrm{e}^{-}+2 \mathrm{O}_{2} \rightarrow \mathrm{O}_{2}^{-}+\mathrm{O}_{2}$ & $6 \times 10^{-39} T_{e}^{-1.0}$ & & -0.43 & {$[25]$} \\
\hline $\mathrm{G}_{13}$ & $\mathrm{O}_{2}^{-}+\mathrm{O}_{4}^{+} \rightarrow 3 \mathrm{O}_{2}$ & $1 \times 10^{-13}$ & & -11.64 & {$[25]$} \\
\hline $\mathrm{G}_{14}$ & $\mathrm{O}_{2}^{-}+\mathrm{O}_{4}^{+}+\mathrm{M} \rightarrow 3 \mathrm{O}_{2}+\mathrm{M}$ & $3.12 \times 10^{-31} T_{g}^{-2.5}$ & & & {$[25]^{b}$} \\
\hline $\mathrm{G}_{15}$ & $\mathrm{O}_{2}^{-}+\mathrm{O}_{2}^{+}+\mathrm{M} \rightarrow 2 \mathrm{O}_{2}+\mathrm{M}$ & $3.12 \times 10^{-31} T_{g}^{-2.5}$ & & & {$[25]^{b}$} \\
\hline $\mathrm{G}_{16}$ & $\mathrm{e}^{-}+\mathrm{O}_{2} \rightarrow \mathrm{e}^{-}+2 \mathrm{O}$ & BOLSIG + & 5.58 & & {$[45]$} \\
\hline $\mathrm{G}_{17}$ & $\mathrm{e}^{-}+\mathrm{O}_{2} \rightarrow \mathrm{e}^{-}+2 \mathrm{O}$ & BOLSIG+ & 8.4 & -2.82 & {$[45]^{c}$} \\
\hline $\mathrm{G}_{18}$ & $\mathrm{e}^{-}+\mathrm{O}_{2} \rightarrow \mathrm{O}^{-}+\mathrm{O}$ & BOLSIG+ & 3.6 & & {$[45]$} \\
\hline $\mathrm{G}_{19}$ & $\mathrm{O}^{-}+\mathrm{O}_{2}^{+} \rightarrow \mathrm{O}+\mathrm{O}_{2}$ & $3.46 \times 10^{-12} T_{g}^{-0.5}$ & & -10.61 & {$[45]$} \\
\hline $\mathrm{G}_{20}$ & $\mathrm{e}^{-}+\mathrm{N}_{2} \rightarrow \mathrm{e}^{-}+\mathrm{N}_{2}$ & BOLSIG+ & 1.0 & -1.0 & {$[45]^{d}$} \\
\hline $\mathrm{G}_{21}$ & $\mathrm{e}^{-}+\mathrm{O}_{2} \rightarrow \mathrm{e}^{-}+\mathrm{O}_{2}$ & BOLSIG+ & 1.0 & -1.0 & {$[45]^{d}$} \\
\hline
\end{tabular}

Table 3.1: Air plasma gas phase reaction mechanism.

Figure 3.1 shows the reaction rate coefficients for all electron impact reactions computed using BOLSIG+. The symbols are data obtained from BOLSIG+ [45], and the lines are curve-fits that are used in the plasma model. The Arrhenius form for reaction rate coefficients did not give satisfactory fits 


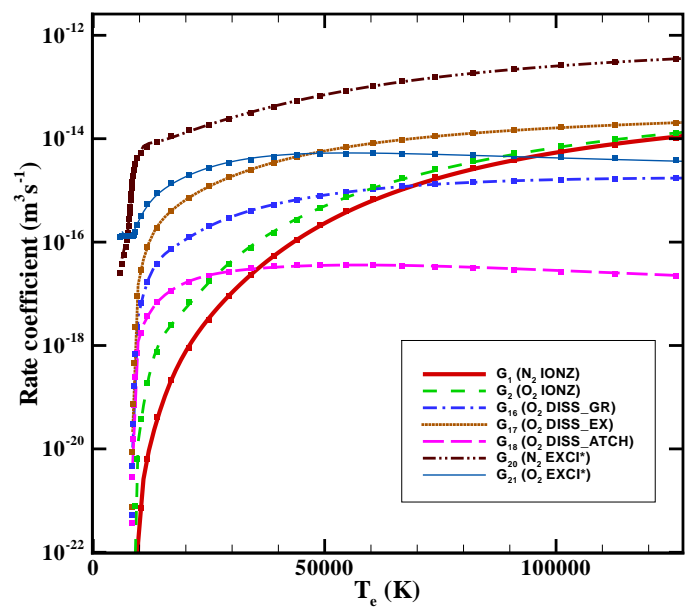

Figure 3.1: Electron impact ionization, excitation, dissociation and dissociative attachment rate coefficients in the air plasma model as a function of the electron temperature.

for the data from BOLSIG+, and hence polynomial fits are used. Appendix A describes the procedure for determining the reaction rate coefficients as a function of the electron temperature using BOLSIG+. These reactions include electron impact ionization reactions $\mathrm{G}_{1}$ and $\mathrm{G}_{2}$, dissociation reactions $\mathrm{G}_{16}$ and $\mathrm{G}_{17}$, and dissociative attachment reaction $\mathrm{G}_{18} \cdot \mathrm{G}_{16}$ and $\mathrm{G}_{17}$ represent electron impact dissociation of $\mathrm{O}_{2}$ that were included in the EEDF calculations and hence added to the air plasma chemistry model for consistency. The dissociative attachment of $\mathrm{O}_{2}$ is the primary source of $\mathrm{O}^{-}$ion production in the discharge, and one of the principal aims of this work is to study the importance of negative ions in the air plasma discharge. $\mathrm{G}_{19}$ provides a channel of destruction of $\mathrm{O}^{-}$ions through two body ion-ion recombination that was in the original mechanism of Kossyi et al. [25]. 
While the excited states of $\mathrm{N}_{2}$ and $\mathrm{O}_{2}$ are not explicitly included in the present model, their role in affecting the EEDF and consequently the electron energetics in the discharge is accounted for. To calculate the electron energy inelastic loss term due to the rotational, vibrational and electronic excitation of ground state $\mathrm{N}_{2}$ and $\mathrm{O}_{2}$, we have included additional reactions $\mathrm{G}_{20}$ and $\mathrm{G}_{21}$. These are "lumped" reactions that cause no net species production and act only as a sink of electron energy. Reaction $\mathrm{G}_{20}$ accounts for the loss of electron energy to produce $\mathrm{N}_{2}$ excited states $\mathrm{N}_{2}$ (rotational), $\mathrm{N}_{2}(\mathrm{X}, \mathrm{v}=1-8), \mathrm{N}_{2}\left(\mathrm{~A}^{3} \Sigma_{u}^{+}\right)$, $\mathrm{N}_{2}\left(\mathrm{~B}^{3} \Pi_{g}\right), \mathrm{N}_{2}\left(\mathrm{~W}^{3} \Delta_{u}\right), \mathrm{N}_{2}\left(\mathrm{~B}^{\prime 3} \Sigma_{u}^{-}\right), \mathrm{N}_{2}\left(\mathrm{a}^{\prime 1} \Sigma_{u}^{-}\right), \mathrm{N}_{2}\left(\mathrm{a}^{1} \Pi_{g}\right), \mathrm{N}_{2}\left(\mathrm{w}^{1} \Delta_{u}\right), \mathrm{N}_{2}\left(\mathrm{C}^{3} \Pi_{u}\right)$, $\mathrm{N}_{2}\left(\mathrm{E}^{3} \Sigma_{g}^{+}\right), \mathrm{N}_{2}\left(\mathrm{a}^{\prime \prime 1} \Sigma_{g}^{+}\right)$, and sum of singlet states [61], for which integral cross section data is obtained from $[62,61]$. Reaction $\mathrm{G}_{21}$ accounts for the loss of electron energy to produce $\mathrm{O}_{2}$ excited states $\mathrm{O}_{2}$ (rotational), $\mathrm{O}_{2}$ (vsum) [63], $\mathrm{O}_{2}\left(\mathrm{a}^{1} \Delta_{g}\right), \mathrm{O}_{2}\left(\mathrm{~b}^{1} \Sigma_{u}^{+}\right)$, and $\mathrm{O}_{2}(4.5)$, with cross section data from $[64,63]$. The integral cross section data is listed in Appendix B. The electron temperature dependent reaction rate coefficients for $\mathrm{G}_{20}$ and $\mathrm{G}_{21}$ are obtained using BOLSIG+ using the same procedure as for the other electron impact reactions in the mechanism (see Appendix A). For the surface chemistry, we assume that ions and radicals that hit solid surfaces return to the plasma as their parent neutral molecule. The surface reactions included in the model are listed in Table 3.2. A unity sticking coefficient is used for all excited neutrals, radicals, and ion species impacting the surfaces.

The electron mobility is also obtained from BOLSIG+ [45] and can be tabulated as a function of the electron temperature. Figure 3.2 shows the reduced electron mobility $\mu_{e} N_{g}$ as a function of the electron temperature. 


\begin{tabular}{|c|c|c|}
\hline & Reaction & Sticking Coefficient \\
\hline $\mathrm{S}_{1}$ & $\mathrm{e}^{-} \rightarrow$ & 1 \\
$\mathrm{~S}_{2}$ & $\mathrm{~N}_{2}^{+} \rightarrow \mathrm{N}_{2}$ & 1 \\
$\mathrm{~S}_{3}$ & $\mathrm{~N}_{4}^{+} \rightarrow 2 \mathrm{~N}_{2}$ & 1 \\
$\mathrm{~S}_{4}$ & $\mathrm{O} \rightarrow 0.5 \mathrm{O}_{2}$ & 1 \\
$\mathrm{~S}_{5}$ & $\mathrm{O}_{2}^{+} \rightarrow \mathrm{O}_{2}$ & 1 \\
$\mathrm{~S}_{6}$ & $\mathrm{O}_{4}^{+} \rightarrow 2 \mathrm{O}_{2}$ & 1 \\
$\mathrm{~S}_{7}$ & $\mathrm{O}_{2}^{-} \rightarrow \mathrm{O}_{2}$ & 1 \\
$\mathrm{~S}_{8}$ & $\mathrm{O}^{-} \rightarrow 0.5 \mathrm{O}_{2}$ & 1 \\
\hline
\end{tabular}

Table 3.2: Air plasma surface reactions.

The ion mobility data is obtained from the literature: $\mathrm{N}_{2}^{+}, \mathrm{O}_{2}^{+}$and $\mathrm{O}^{-}$data from [65], $\mathrm{N}_{4}^{+}$data from [66] (same is assumed for $\mathrm{O}_{4}^{+}$and $\mathrm{O}_{2}^{+} \mathrm{N}_{2}$ ), and $\mathrm{O}_{2}^{-}$data from [67]. The standard mobility value $\mu_{0}$ is defined to obtain the mobility $\mu=N_{0} \mu_{0} / N_{g}$, where $N_{g}$ is the sum of densities of $\mathrm{N}_{2}$ and $\mathrm{O}_{2}$ and $N_{0}$ is Loschmidt's number. For positive and negative ions, the ion mobility tabulated as a function of the reduced electric field $\left(E / N_{g}\right)$ is shown in Fig. 3.3.

For electrons, negative ions and positive ions, the species diffusion coefficient and the mobility used in the drift-diffusion approximation in (2.3) are related by Einstein's relation

$$
D_{k}=\frac{k_{B} T_{k}}{e Z_{k}} \mu_{k}
$$

For neutral species, the diffusion coefficient is found using the formula

$$
D_{k}=\frac{k_{B} T_{g}}{m \bar{\nu}_{k, k_{b}}}
$$

where the momentum transfer collision frequency is $\bar{\nu}_{k, k_{b}}=n_{k} \overline{\bar{\sigma}}_{k, k_{b}} \bar{g}_{k, k_{b}}$ and $\overline{\bar{\sigma}}_{k, k_{b}}$ is the momentum transfer cross section obtained from Lennard-Jones collision diameters [68] listed in Table 3.3, and $\bar{g}_{k, k_{b}}$ is the average relative 


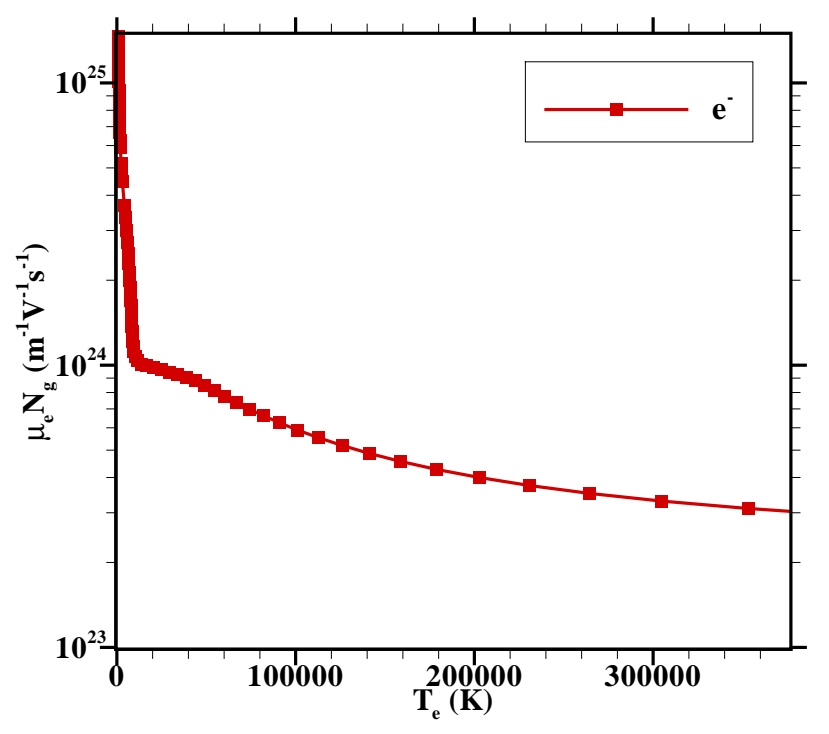

Figure 3.2: Mobility data for electrons as a function of electron temperature obtained from BOLSIG+.

molecular speed of species $k$. The species transport properties are summarized in Table 3.4 .

\begin{tabular}{|c|c|c|c|}
\hline Species & L-J Collision Diameter $(\AA)$ & $\overline{\bar{\sigma}}_{k, k_{b}}\left(\AA^{2}\right)$ & Ref. \\
\hline $\mathrm{N}_{2}$ & 3.458 & 38.98 & {$[68]$} \\
$\mathrm{O}_{2}$ & 2.750 & 31.54 & {$[68]$} \\
$\mathrm{O}$ & 3.621 & 40.81 & {$[68]$} \\
\hline
\end{tabular}

Table 3.3: Neutral species transport properties.

The secondary electron emission coefficients $\gamma_{k}$ appearing in the electron flux boundary conditions, Eqs. (2.8) and (2.16) are chosen according to the empirical correlation $\gamma_{k} \approx 0.016\left(\varepsilon_{i z}-2 \varepsilon_{\phi}\right)[69,1]$ for clean surfaces, where $\varepsilon_{i z}$ is the ionization potential of the impacting ion and $\varepsilon_{\phi}$ is the work function of the surface. Assuming a work function value $\varepsilon_{\phi}=4.5 \mathrm{eV}$ (for tungsten), 


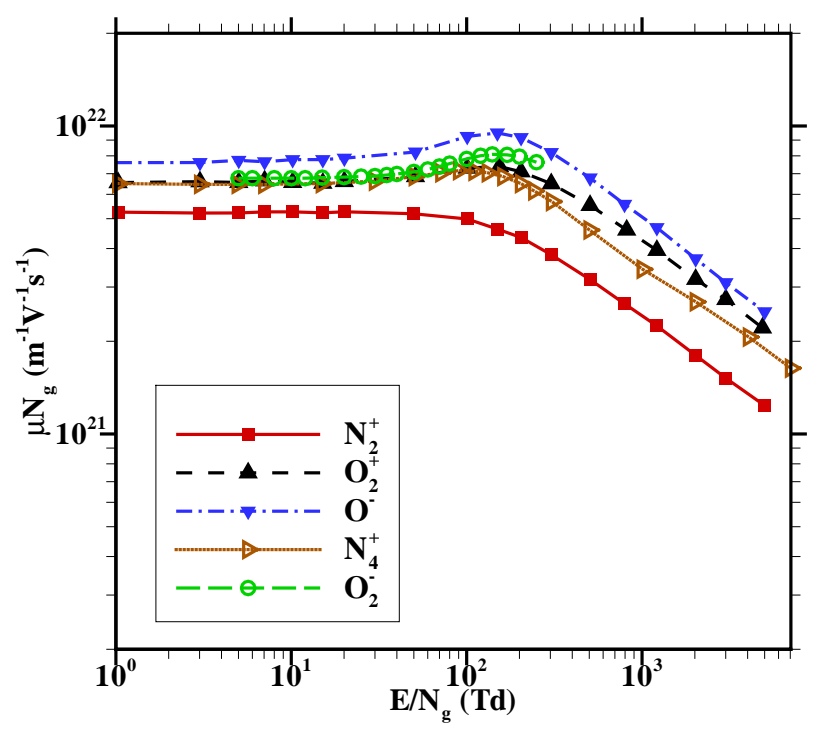

Figure 3.3: Ion mobility data obtained from the literature as a function of the reduced electric field.

the secondary electron emission coefficients are evaluated as $\sim 0.1$ for $\mathrm{N}_{2}^{+}$and $\sim 0.05$ for $\mathrm{O}_{2}^{+}$ions. For all other species in the model, the secondary electron emission coefficient is assumed to be zero. For the results presented in this paper, the model was found to be most sensitive to the secondary electron emission coefficient of $\mathrm{N}_{2}^{+}$.

The energy of secondary electrons entering the plasma is computed as follows. For electrons at the top of the conduction band of the solid, the electron energy released due to secondary emission is $\Delta E_{e}^{s e} \mid \max =\left(\varepsilon_{i z}-\right.$ $\left.2 \varepsilon_{\phi}\right)$ [1]. If both electrons are in the center of the conduction band, the electron energy released is $\Delta E_{e}^{s e}=\left(\varepsilon_{i z}-2 \varepsilon_{\phi}-\varepsilon_{F}\right)$. Assuming values of work function $\varepsilon_{\phi}=4.5 \mathrm{eV}$ and Fermi energy $\varepsilon_{F}=4.5 \mathrm{eV}$, the energy of the secondary 


\begin{tabular}{|c|c|c|c|}
\hline & Species & Transport Property & Ref. \\
\hline 1 & $\mathrm{e}^{-}$ & Mobility & BOLSIG+ [45] (Fig. 3.2) \\
2 & $\mathrm{~N}_{2}$ & Momentum transfer cross section & {$[68]$ (Table 3.3) } \\
3 & $\mathrm{O}_{2}$ & Momentum transfer cross section & [68] (Table 3.3) \\
4 & $\mathrm{O}$ & Momentum transfer cross section & [68] (Table 3.3) \\
5 & $\mathrm{~N}_{2}^{+}$ & Mobility & {$[65]$ (Fig. 3.3) } \\
6 & $\mathrm{O}_{2}^{+}$ & Mobility & [65] (Fig. 3.3) \\
7 & $\mathrm{O}^{-}$ & Mobility & [65] (Fig. 3.3) \\
8 & $\mathrm{~N}_{4}^{+}$ & Mobility & same as $\mathrm{N}_{4}^{+}$ \\
9 & $\mathrm{O}_{4}^{+}$ & Mobility & same as $\mathrm{N}_{4}^{+}$ \\
10 & $\mathrm{O}_{2}^{+} \mathrm{N}_{2}^{+}$ & Mobility & [67] (Fig. 3.3) \\
11 & $\mathrm{O}_{2}^{-}$ & Mobility & \\
\hline
\end{tabular}

Table 3.4: Summary of transport property data for all species in the air plasma chemistry model.

electrons entering the gas due to impact of $\mathrm{N}_{2}^{+}$is estimated to be about 2.1 $\mathrm{eV}$, which was the value used in the simulations.

\subsection{Air plasma model validation results}

The air plasma model is validated using experimental data from the literature. The experiments were performed by Lisovskiy et al. [70] in a parallelplate direct-current glow discharge environment. The discharge geometry and the computational mesh used for the simulations are shown in Fig. 3.4. The discharge is formed between circular plate electrodes of radius $80 \mathrm{~mm}$ separated by a distance $\mathrm{L}$, and is radially bounded by a quartz dielectric tube of inner radius $50 \mathrm{~mm}$. The thickness of the dielectric is assumed to be 3 $\mathrm{mm}$. The computational mesh has three subdomains containing a total of 10600 cells. The plasma subdomain contains 8000 cells, bounded by a solid subdomain for the quartz wall and a dielectric (air) with vacuum permittivity 
outside.

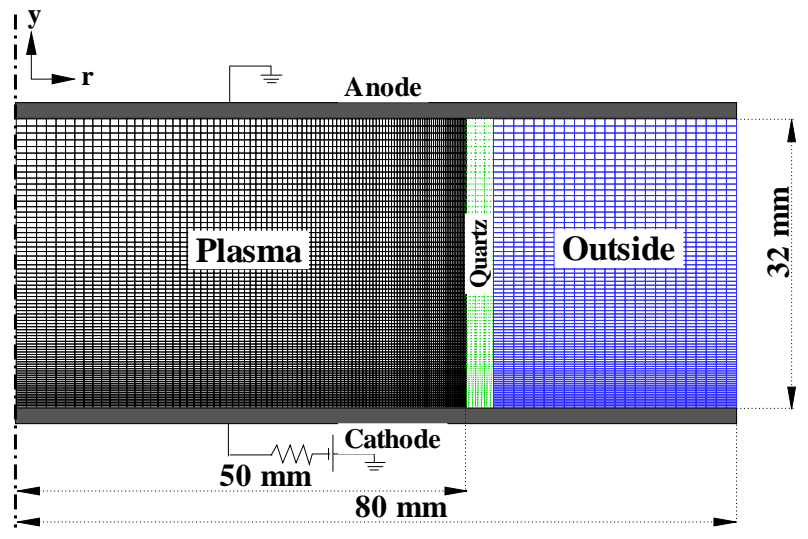

Figure 3.4: Geometry and mesh used for the validation of the air plasma model. The mesh contains a total of 10160 cells, with 8000 cells in the plasma subdomain.

Poisson's equation is solved on all three subdomains, and the species conservation and electron energy equations are solved on the plasma subdomain alone. Note that the Poisson's equation reduces to the Laplace equation (in the absence of space charge) within the quartz tube and the outer air subdomains. A fixed gas temperature of $300 \mathrm{~K}$ is applied in all subdomains (a gas energy equation is not solved in the plasma) since it is expected that gas heating is not important under these conditions. The experimental studies by Lisovskiy et al. [70] were conducted for glow discharges in air for pressures of $\sim 1$ Torr, which matches the pressure range of interest for the application of glow discharges as high-speed flow actuators. Experimental results for an 
inter-electrode distance $\mathrm{L}=32 \mathrm{~mm}$ and pressure $\mathrm{p}=600 \mathrm{~m}$ Torr are chosen for comparison/validation in this study.

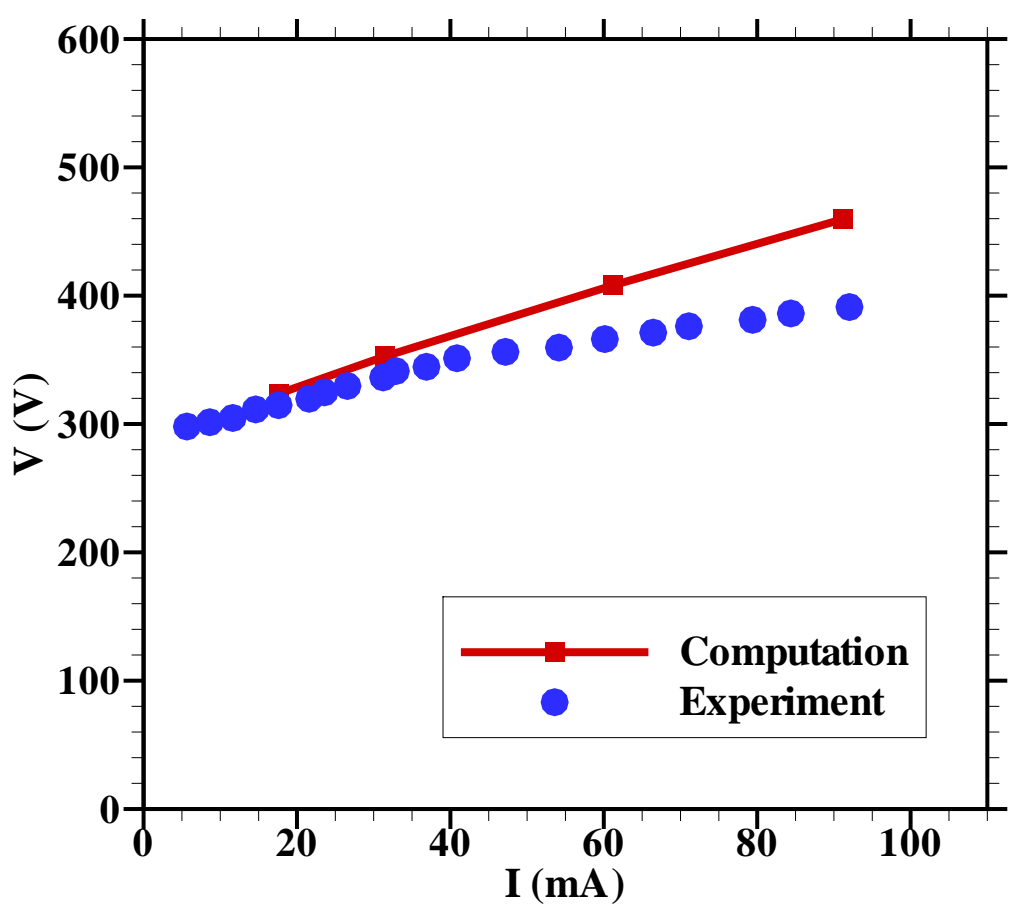

Figure 3.5: Comparison of current-voltage characteristic with experimental data. The pressure is $600 \mathrm{mTorr}$, and inter-electrode distance is $32 \mathrm{~mm}$.

Figure 3.5 shows the computed voltage-current (V-I) characteristic and corresponding experimental results. In the experimental studies a series ballast resistance of $1.5 \mathrm{k} \Omega$ was used in the external circuit to stabilize the discharge. From Fig. 3.5 we see that the computational model shows good agreement with the experimental results particularly at lower currents, with the discharge voltage being increasingly over-predicted as the current increases. The experimental V-I curve shows a gradual decrease in slope (differential re- 
sistivity) with increasing current. This decrease in the differential resistivity is not predicted well by the computational model. A possible explanation for this discrepancy is the dependence of the secondary electron emission coefficient on the ion impact energy (related to the reduced electric field) at the cathode surface. Since the present model assumes a constant secondary electron emission coefficient, the computed V-I curve has a single slope (nearly constant differential resistivity) over the range of discharge currents studied. Previous work from our group has shown the importance of using a variable secondary electron emission coefficient that depends on the reduced electric field for an argon microhollow cathode discharge [71]. The variable secondary electron emission coefficient increases with increasing reduced electric field at the cathode surface [72] (i.e. increasing ion impact energies).

We have performed additional calculations in the air glow discharge to show that increasing the constant secondary electron emission coefficient results in a decrease in the discharge voltage for a given discharge current. These calculations are shown in Fig. 3.6. The choice of secondary electron emission coefficients based on the empirical correlation $\left(\gamma_{\mathrm{N}_{2}^{+}}=0.1\right.$ and $\gamma_{\mathrm{O}_{2}^{+}}=$ 0.05) discussed in Section 3.1 is found to provide a good match at the low current range, which matches the range of currents studied for the actuator simulations in Chapters 4 and 6. For lower values of the secondary electron emission coefficients, the computed V-I curve matches neither the slope nor the values from the experimental dataset. As the secondary electron emission coefficients are increased, the slope of the $\mathrm{V}$-I characteristic is found to decrease and is closer to the slope from the experiments at higher currents. Hence it 


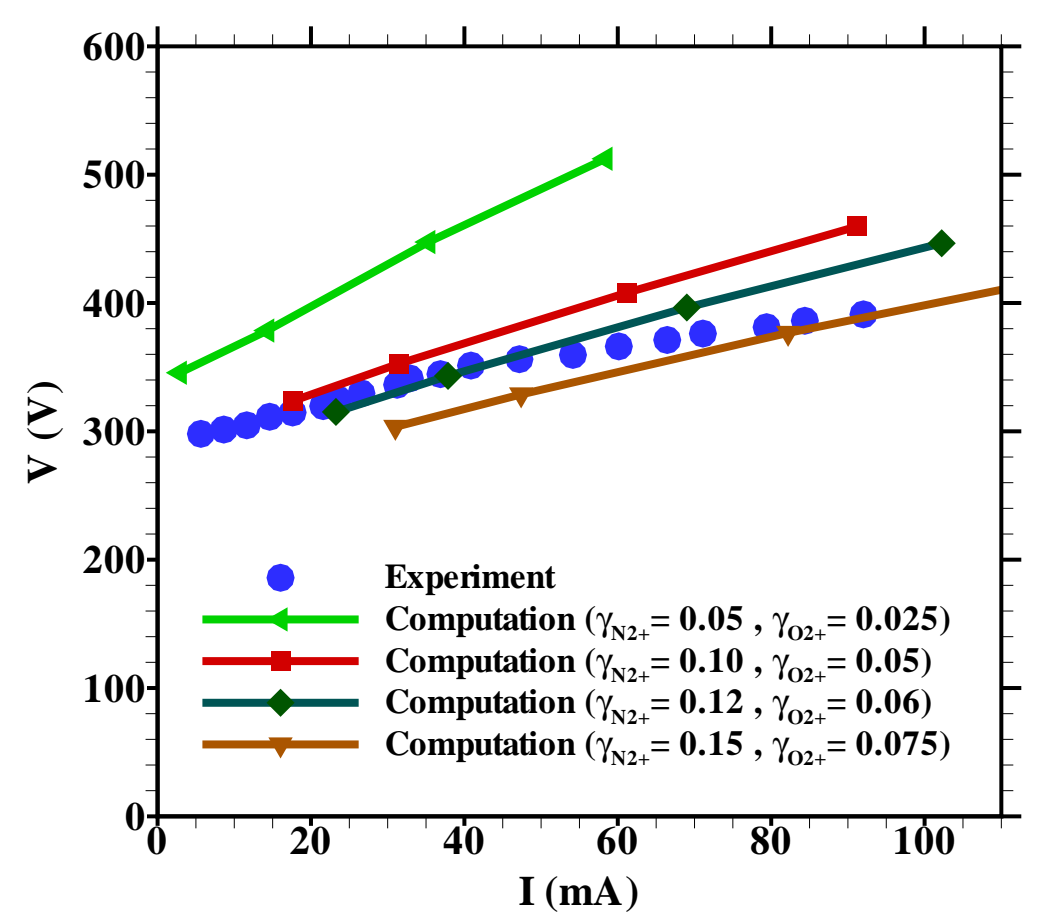

Figure 3.6: Comparison of current-voltage characteristic with experimental data with various values of secondary electron emission coefficient for $\mathrm{N}_{2}^{+}$and $\mathrm{O}_{2}^{+}$. The pressure is $600 \mathrm{mTorr}$, and inter-electrode distance is $32 \mathrm{~mm}$.

is plausible that if the secondary electron emission coefficient increased with increasing ion impact energy at the cathode surface (owing to increased sheath potential drop), the slope of the V-I curve would provide better agreement with the experimental data at higher currents. Consequently, we expect improved treatment of secondary electron emission model using a variable coefficient formulation would provide improved prediction of the V-I curve over the entire discharge current range studied.

The electron temperature and total positive ion density (sum of all pos- 


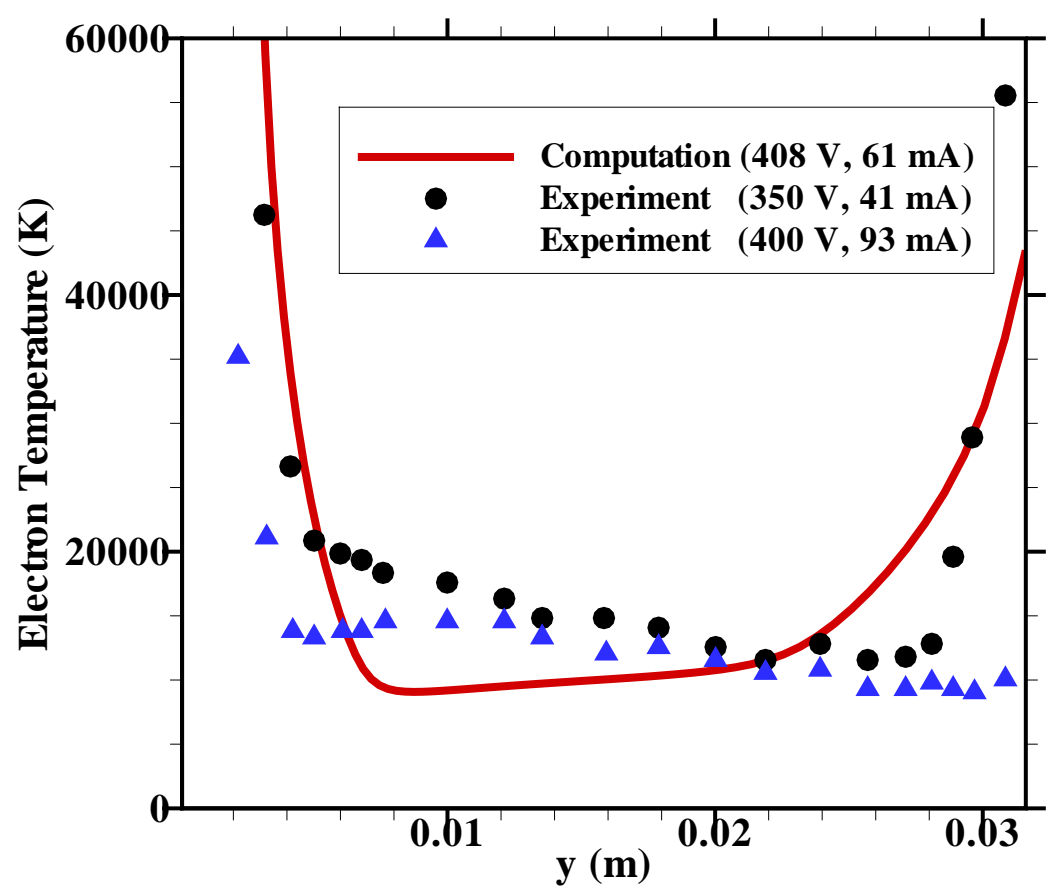

Figure 3.7: Comparison of the computed axial electron temperature (units: $\mathrm{K})$ with experimental data. The cathode is at $\mathrm{y}=0$ and the anode is at $\mathrm{y}=$ $32 \mathrm{~mm}$. The applied voltage at the cathode is $-408 \mathrm{~V}$.

itive ions) profiles along the discharge centerline (axis) are shown in Figs. 3.7 and 3.8 , respectively $(\mathrm{y}=0 \mathrm{~mm}$ corresponds to the cathode and $\mathrm{y}=32 \mathrm{~mm}$ to the anode). The figures show experimental profile data from Lisovskiy et al. [70] for two different operating conditions: 1) discharge voltage drop of $350 \mathrm{~V}$ resulting in discharge current of $41 \mathrm{~mA}$ and 2) discharge voltage drop of $400 \mathrm{~V}$ resulting in a discharge current of $93 \mathrm{~mA}$. The computational results are shown for a case with discharge voltage drop of $408 \mathrm{~V}$ which results in a computed discharge current of $61 \mathrm{~mA}$. The discharge condition for the computation result 


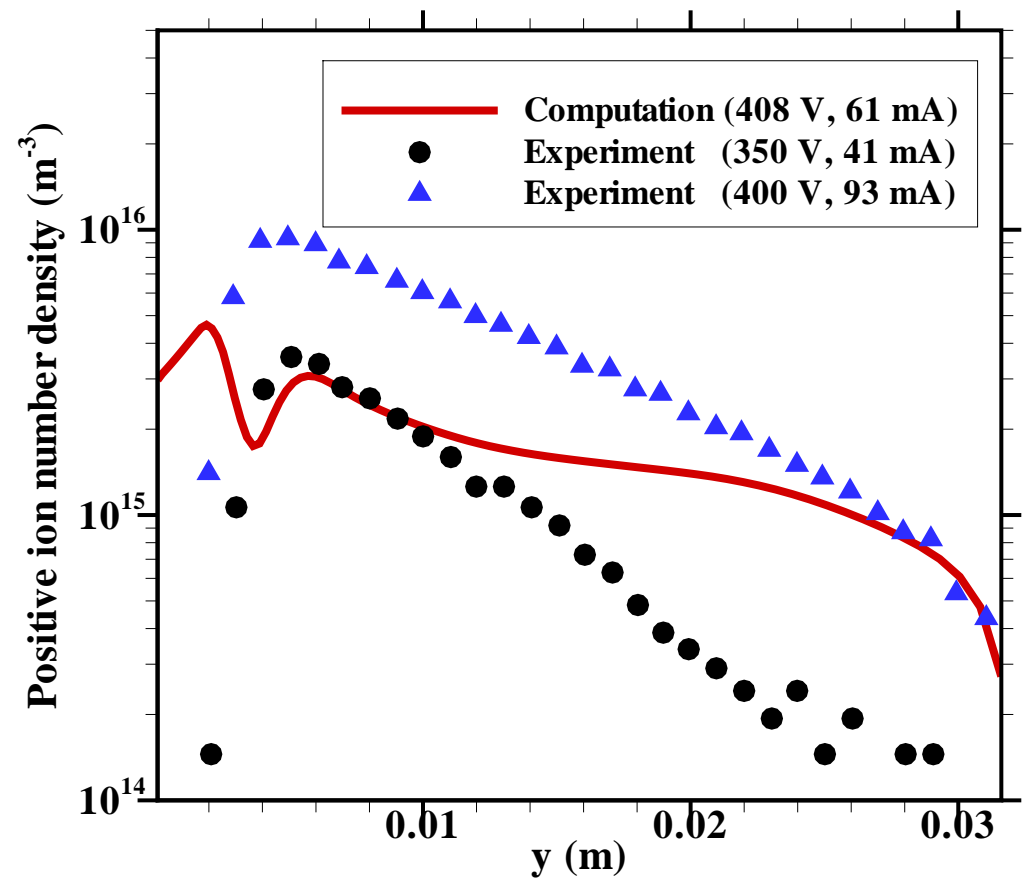

Figure 3.8: Comparison of the computed axial total positive ion density (units: $\mathrm{m}^{-3}$ ) with experimental data. The cathode is at $\mathrm{y}=0$ and the anode is at $\mathrm{y}$ $=32 \mathrm{~mm}$. The applied voltage at the cathode is $-408 \mathrm{~V}$.

is therefore bracketed by the two experimental conditions shown. The electron temperature curve in Fig. 3.7 shows good qualitative agreement with the experimental measurements. The major features of a relatively uniform electron temperature in the bulk plasma and increase in temperature in the cathode and anode sheath regions are captured well by the model. Quantitative discrepancies observed in the computational electron temperature profile are no more than about $50 \%$. The total positive ion density on the axis is compared with experimental values in Fig. 3.8. The peak computed values differ from 
the experimental measurements by a factor of 2 . Two number density peaks are observed from the computation results; one peak in the cathode sheath and the second peak in the bulk plasma. In the experiments, only one number density peak is observed for both discharge conditions in Fig. 3.8. However, the experimental ion number density profile in the immediate vicinity of the cathode surface is not available perhaps due to experimental limitations, and hence the detailed comparison of this profile with the computational results in the cathode sheath region is not possible.

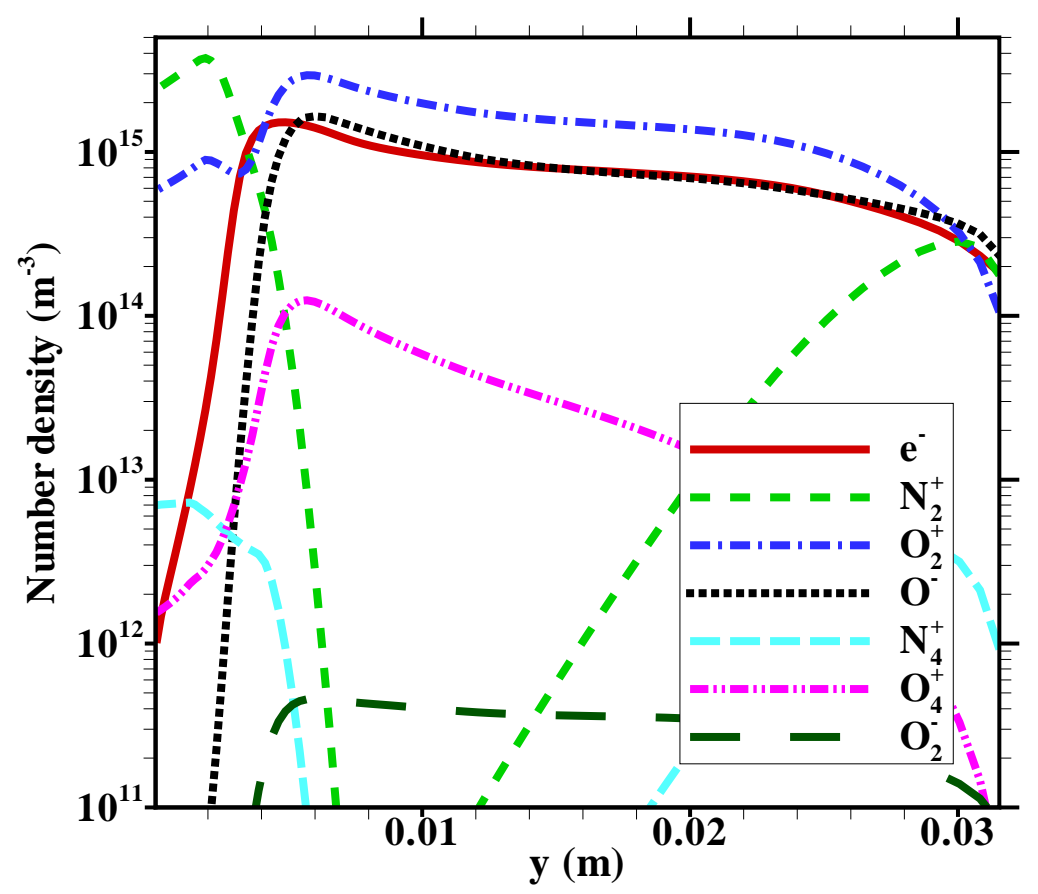

Figure 3.9: Number densities (units: $\mathrm{m}^{-3}$ ) of charged species in the air plasma model along the axis. The cathode is at $\mathrm{y}=0$ and the anode is at $\mathrm{y}=32 \mathrm{~mm}$. The applied voltage at the cathode is $-408 \mathrm{~V}$. 
Figure 3.9 presents computational results for charged species density profiles along the axis of the discharge. The dominant positive ion in the bulk plasma is $\mathrm{O}_{2}^{+}$. The dominant positive ion species in the cathode sheath is $\mathrm{N}_{2}^{+}$ with $\mathrm{O}_{2}^{+}$being less important but significant. As a consequence of the dominance of $\mathrm{N}_{2}^{+}$in the cathode sheath, the direct-current discharge model is most sensitive to the secondary electron emission coefficient value of $\mathrm{N}_{2}^{+}$, as noted previously in Section 3.1. In the bulk plasma, the $\mathrm{N}_{2}^{+}$ion density decreases sharply to negligible values followed by a small increase as the anode boundary is approached. The profile of $\mathrm{N}_{2}^{+}$ions in the discharge can be attributed to the following reasons. At the relatively low electron temperatures of $\sim 1 \mathrm{eV}$ observed in the bulk plasma, the ionization rate of $\mathrm{O}_{2}$ to form $\mathrm{O}_{2}^{+}$(reaction $\mathrm{G}_{2}$ ) is relatively high compared to the equivalent reaction for the formation of $\mathrm{N}_{2}^{+}$(reaction $\mathrm{G}_{1}$ ) (see Fig. 3.1). Besides, $\mathrm{N}_{2}^{+}$formed by reaction $\mathrm{G}_{1}$ is also converted to $\mathrm{O}_{2}^{+}$by reaction $\mathrm{G}_{5}$. In the cathode and anode sheath regions the increase in the electron temperatures results in an increase in the $\mathrm{N}_{2}^{+}$ion production rate relative to the $\mathrm{O}_{2}^{+}$ion production rates through reactions $\mathrm{G}_{1}$ and $\mathrm{G}_{2}$, respectively. The two positive ion density peaks observed in Fig. 3.8 correspond to the peak density of $\mathrm{N}_{2}^{+}$in the cathode sheath and the $\mathrm{O}_{2}^{+}$peak density in the bulk plasma. Density profiles of cluster ions $\mathrm{O}_{4}^{+}$and $\mathrm{N}_{4}^{+}$, are also shown in Fig. 3.9. Both cluster ions have significantly lower density than the dominant dimer ions $\mathrm{O}_{2}^{+}$and $\mathrm{N}_{2}^{+}$in the bulk plasma and sheath regions. The $\mathrm{N}_{4}^{+}$ion is formed by three-body ion conversion reaction $\mathrm{G}_{3}$, while $\mathrm{O}_{4}^{+}$is formed by reactions $\mathrm{G}_{8}$ and $\mathrm{G}_{9}$. In reaction $\mathrm{G}_{8}$, the $\mathrm{O}_{4}^{+}$ion is formed through the intermediate ion complex $\mathrm{O}_{2}^{+} \mathrm{N}_{2}$ which is in turn produced by the three- 
body reaction $\mathrm{G}_{6}$. The density of $\mathrm{O}_{2}^{+} \mathrm{N}_{2}$ is about $10^{10} \mathrm{~m}^{-3}$, which is negligible compared to the other ionized species in the plasma and hence does not appear in Fig. 3.9. For the operating pressure of 600 mTorr considered in this study the rate-of-progress of three-body reactions is not high enough to produce the cluster ions. However, at higher pressures (>10 Torr) it is anticipated that cluster ion densities formed by ion conversion can be significant in the discharge [40, 73].

The dominant negative ion in the discharge is $\mathrm{O}^{-}$with negligible $\mathrm{O}_{2}^{-}$ negative ion density. The reason for this behavior is that $\mathrm{O}_{2}^{-}$is produced by three-body reaction $\mathrm{G}_{12}$; while $\mathrm{O}^{-}$is produced by the bimolecular dissociative attachment reaction $\mathrm{G}_{18}$. At higher discharge pressures we again expect that the $\mathrm{O}_{2}^{-}$ion densities will be comparable or even exceed the $\mathrm{O}^{-}$ion densities. The total negative ion density in the bulk plasma is comparable to the electron density. The negative ions are completely excluded from the cathode region owing to the sheath electrostatic field.

Fig. 3.10 shows the profiles of the total positive ion density, total negative ion density and electron density along the axis of the discharge. The dotted line in Fig. 3.10 shows the net charged species density $\left(=n_{\text {positive ions }}-\right.$ $\left.\mathrm{n}_{\text {negative ions }}-\mathrm{n}_{\text {electrons }}\right)$ in the plasma, from which the cathode sheath thickness can be inferred to be about $4 \mathrm{~mm}$. As seen in Fig. 3.10, just outside the cathode sheath edge, an electron-ion plasma region is observed, beyond which the total negative ion density increases to equal the electron density. A pure ion-ion core characteristic of strongly electronegative plasmas is not found; the air plasma under these conditions is therefore only moderately electronegative. 


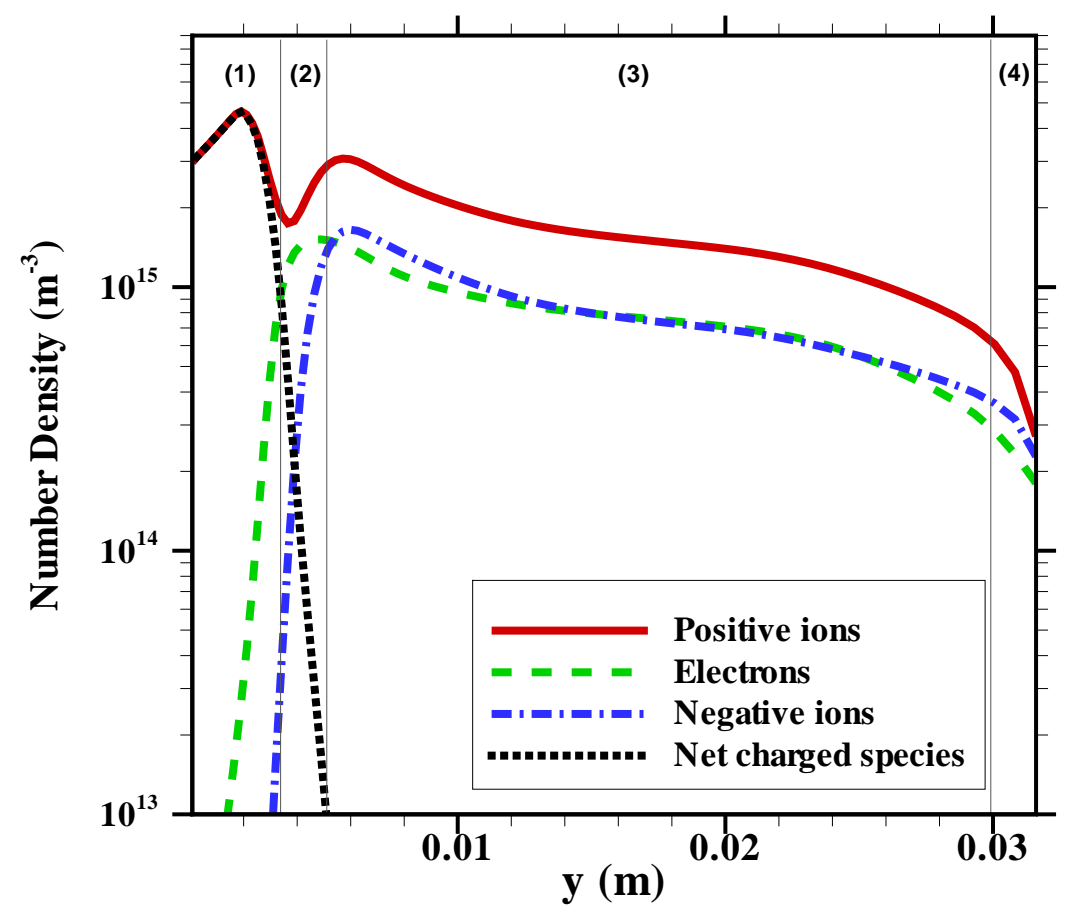

Figure 3.10: Profiles of positive ion, electron, and negative ion density in the air plasma model along the discharge axis. The regions of the plasma are labeled: (1) cathode sheath, (2) electron-positive ion layer, (3) electronpositive ion-negative ion core and (4) anode sheath. The net charged species density is also shown.

The fine structure of electronegative discharges, with a electropositive sheath, an adjacent thin electron-positive ion layer, and electron-positive ion-negative ion core in the bulk plasma, is captured well by the present model. In the vicinity of the anode, the sum of the total negative ion density and the electron density is higher than the positive ion species density resulting in a net negative space charge in the vicinity of the anode. Consequently the anode sheath electrostatic field points away from the anode resulting in an inverted 
anode sheath as shown in Fig. 3.11.

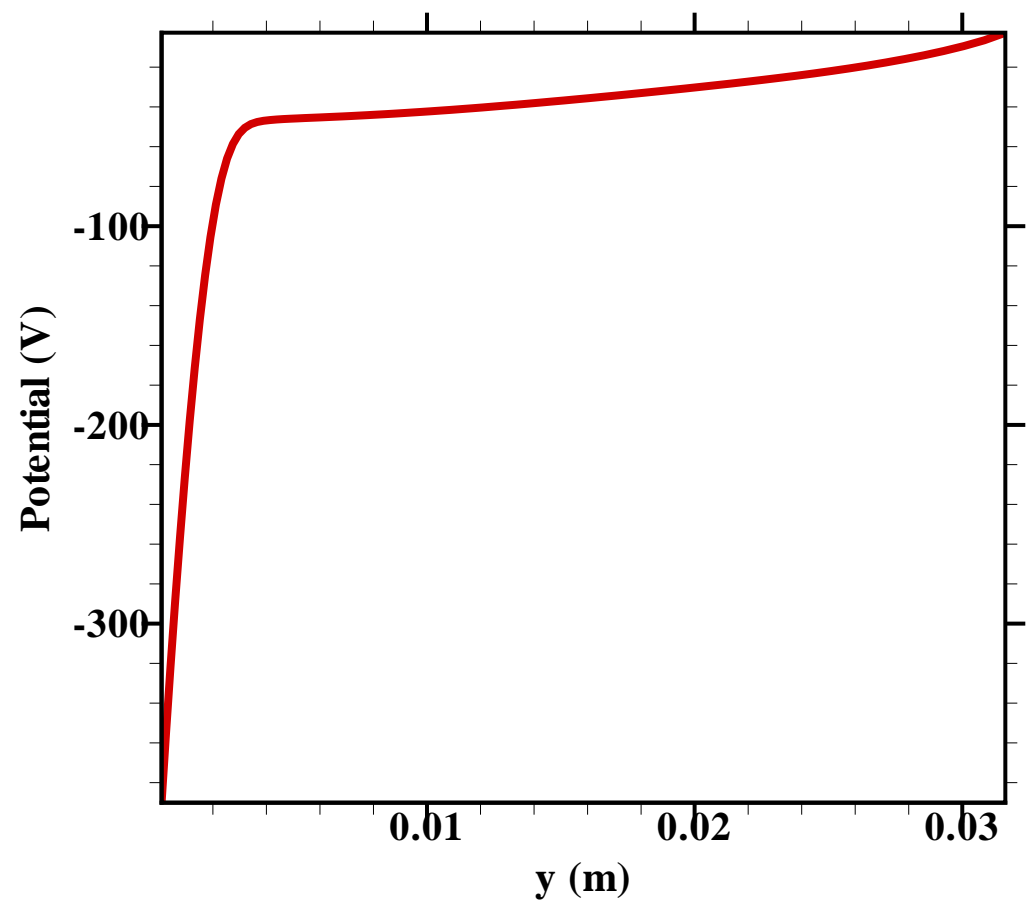

Figure 3.11: Potential (units: V) profile in the air plasma model along the discharge axis. The cathode is at $\mathrm{y}=0$ and the anode is at $\mathrm{y}=32 \mathrm{~mm}$. The applied voltage at the cathode is $-408 \mathrm{~V}$.

An explanation for the formation of an inverted sheath is as follows [74]. In a purely electropositive (no negative ions) discharge at low pressures, a positive current from the anode is preserved by a net negative (electron) charge flux to the anode surface. Under these conditions, a low voltage positive (noninverted) sheath is necessary at the anode to prevent excessive electron flux to the anode surface so as to maintain the necessary current condition at the anode. For electronegative discharges at higher pressures, the electron density 
at the anode sheath edge decreases in proportion to the positive ion density because of the contribution of negative ion in maintaining quasineutrality in the bulk plasma. The significantly lower mobility of negative ions in comparison to the electrons changes the anode sheath potential requirement. An inverted sheath is formed to extract negative ions (and electrons) from the bulk plasma towards the anode and prevent flux of positive ions towards anode so as to maintain the necessary current condition at the anode. With increasing electronegativity of the plasma the magnitude of the inverted anode sheath potential increases. The inverted anode sheath potential also results in electrons gaining energy as they approach the anode, which is consistent with the rise in electron temperature in the anode sheath as shown in Fig. 3.7.

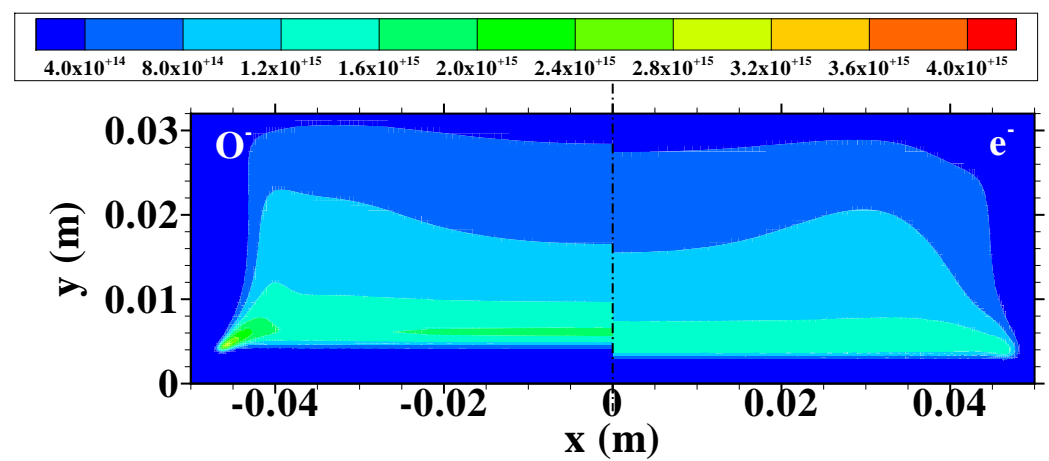

Figure 3.12: Number density contours (units: $\mathrm{m}^{-3}$ ) of dominant negatively charged species (left: $\mathrm{O}^{-}$, right: $\mathrm{e}^{-}$) in the plasma subdomain. The applied voltage at the cathode is $-408 \mathrm{~V}$.

The two-dimensional structure of the discharge is shown in Figs. 3.12 and 3.13 which present number density contours of the major charged species $\left(\mathrm{e}^{-}, \mathrm{O}^{-}, \mathrm{O}_{2}^{+}\right.$and $\left.\mathrm{N}_{2}^{+}\right)$in the plasma subdomain. For all the charged species 


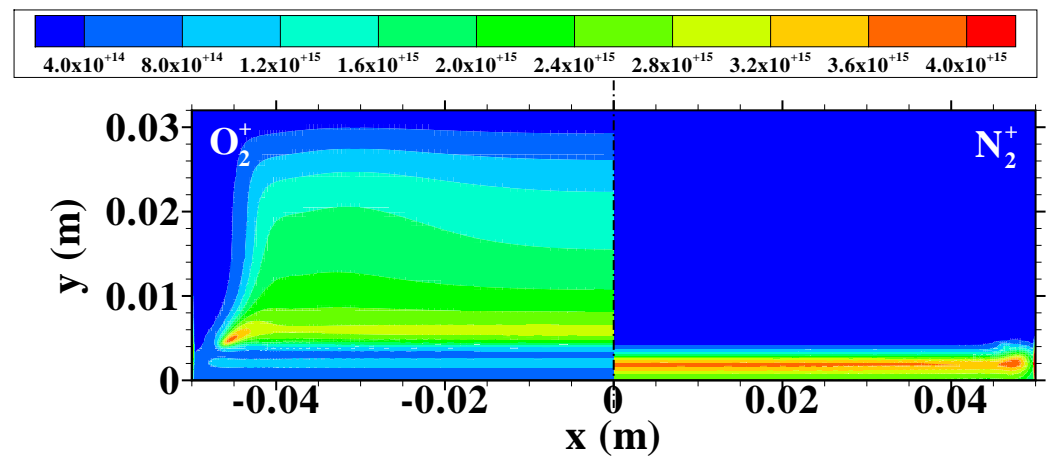

Figure 3.13: Number density contours (units: $\mathrm{m}^{-3}$ ) of dominant positively charged species (left: $\mathrm{O}_{2}^{+}$, right: $\mathrm{N}_{2}^{+}$) in the plasma subdomain. The applied voltage at the cathode is $-408 \mathrm{~V}$.

shown, the peak density is found close to the corner between the outer confining quartz wall and the cathode surface, the discharge therefore has a toroidal ringshaped structure. The toroidal structure of the plasma at low currents was also reported in the experiments by Lisovskiy et al. [70].

Figure 3.14 shows the electrostatic potential and the electron temperature contours in the domain. The electrostatic potential contours are presented for the complete simulation domain including the plasma, quartz and outside subdomains. For clarity, the outline of the quartz boundaries is shown. The electrostatic potential near the quartz wall on the outer side increases uniformly from $-408 \mathrm{~V}$ at the cathode to $0 \mathrm{~V}$ at the anode, which results in the highest electric fields observed in the vicinity of the cathode-quartz boundary intersection. The lower part of the quartz surface therefore acts like an extension of the cathode surface when the plasma is formed, resulting in local peaks in the charged species profiles at this corner of the discharge. 


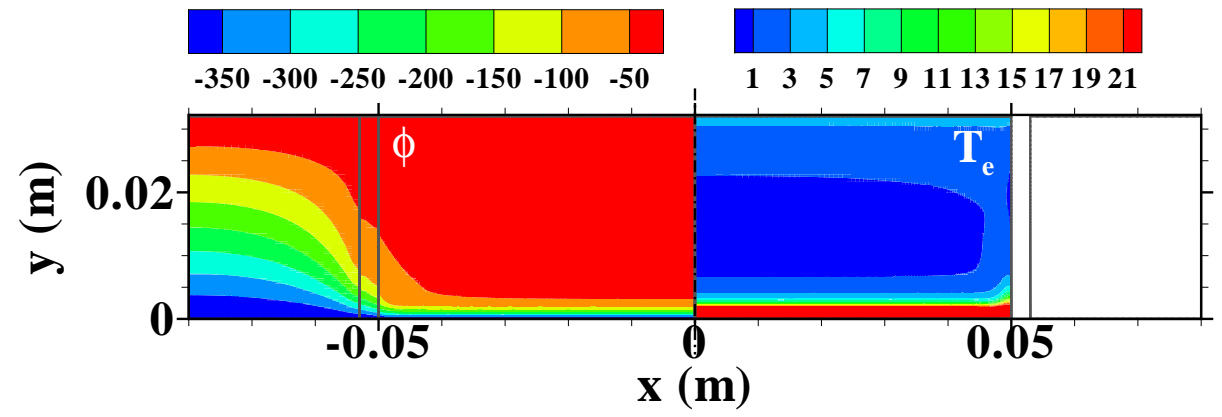

Figure 3.14: Contours of potential (units: V) in the plasma, quartz and outside subdomains and electron temperature (units: eV) in the plasma subdomain. The applied voltage at the cathode is $-408 \mathrm{~V}$.

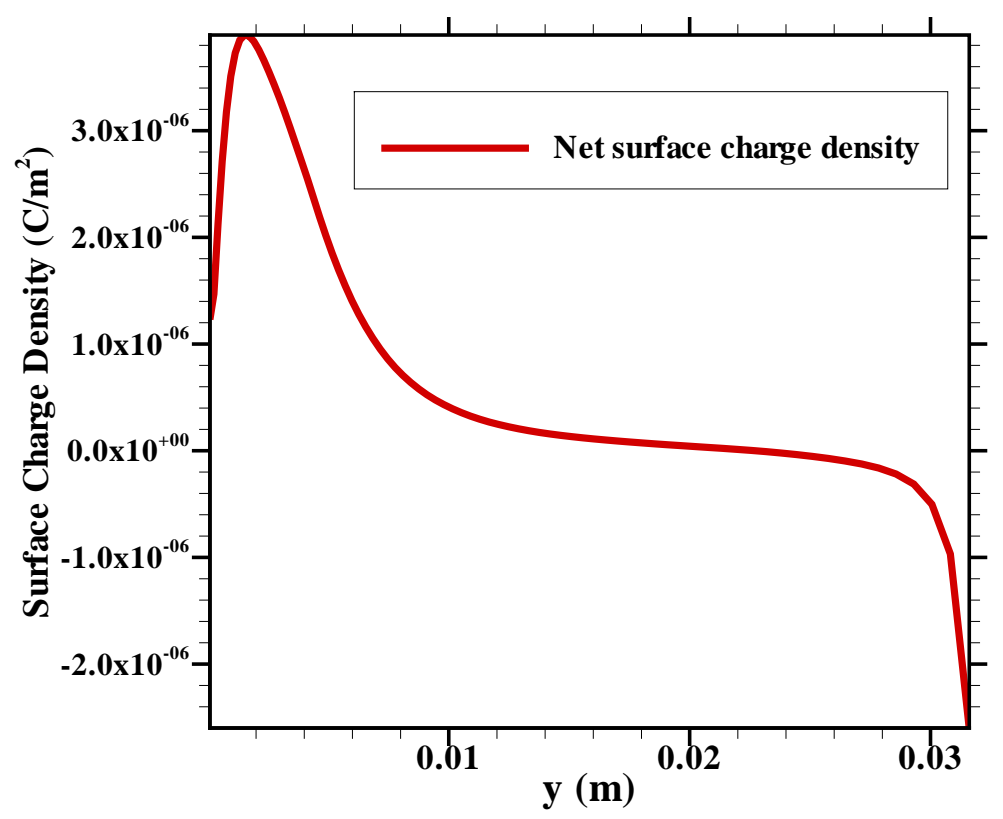

Figure 3.15: Net surface charge density (units: $\mathrm{C} / \mathrm{m}^{2}$ ) on the plasma-quartz dielectric interface. 
The electron temperature is found to be nearly uniform in the bulk plasma region with a value of about $12,000 \mathrm{~K}(\sim 1 \mathrm{eV})$ and rises sharply in the cathode sheath regions adjacent to the cathode surface and the lower part of the quartz dielectric wall (the extended cathode surface).

Fig. 3.15 shows the surface charge density distribution on the plasmadielectric interface. The cathodic nature of the lower side of the quartz results in positive charge accumulation on the dielectric surface. Although the lower part of the quartz dielectric acts like an extension of the cathode, the distinction between the two is that the cathode carries a current while no net current is carried through the dielectric. The positive surface charge trapping on the quartz dielectric surface is merely a requirement to ensure this "no current condition" at the surface.

Finally, we make some additional comments about the air plasma model and results from the simulations. In most electropositive discharges, the principal structural features of the discharge include the cathode sheath and the bulk plasma. The computational mesh for simulating such discharges needs to resolve the cathode sheath and a coarser mesh may be used in the quasineutral bulk plasma region. For the conditions discussed in this study, the air plasma was found to be moderately electronegative, with discharge fine structure including the cathode sheath, the thin electron-positive ion region within the bulk plasma, the larger electron-positive ion-negative ion bulk plasma, and the anode sheath. The mesh resolution requirements are therefore more stringent in the case of air discharges, with a need to resolve the cathode sheath and also additional thin features with the bulk plasma. As shown in Fig. 3.4, 
additional mesh resolution was also required in the corner region between the cathode and the lower surface of the dielectric in order to sufficiently resolve the toroidal ring-shape of the discharge.
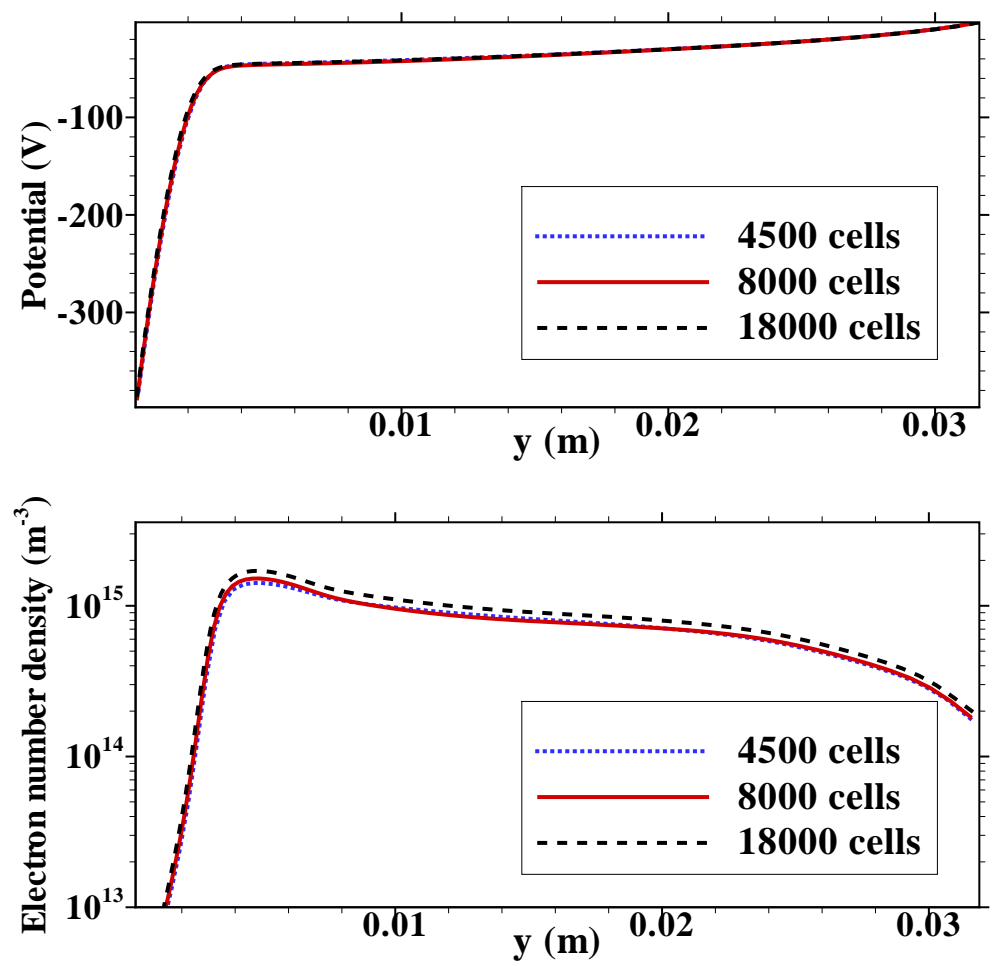

Figure 3.16: Potential (units: V) and electron density (units: $\mathrm{m}^{-3}$ ) profiles in the air plasma model along the discharge axis for varying mesh resolutions in the plasma subdomain. The cathode is at $\mathrm{y}=0$ and the anode is at $\mathrm{y}=32$ $\mathrm{mm}$. The applied voltage at the cathode is $-408 \mathrm{~V}$.

The effect of mesh resolution on the computational results presented in this work is examined in Fig. 3.16. Profiles of potential and electron density along the axis are shown for cell counts of 4500, 8000 and 18000 cells in the plasma subdomain with 60,80 and 120 cells respectively in the axial direction geometrically stretched away from the cathode. The number of cells in the 
quartz and outer subdomains were held fixed (2160 cells) for all three cases. While excellent agreement is observed in the predicted potential profile, the electron density profile is more sensitive to the computational mesh used. Differences in peak electron density magnitudes between the 8000 and 18000 cell cases are approximately $15 \%$.

The fact that the plasma is moderately electronegative under the present conditions and the electronegativity has a strong influence on the discharge potential and electron temperature profiles indicates that a pure nitrogen plasma model is not a good surrogate for an air plasma model as used in several recent modeling studies.

The time-splitting approach used to alleviate temporal stiffness in the model described in Section 2.1.3 was an important factor in obtaining the steady state simulation results. Despite the improved efficiency of the timesplitting approach, about 1-1.5 million electron-Poisson time steps were required to obtain a steady state, which took 3-4 weeks of run time on a highperformance blade server (serial computation) for the current air chemistry model (11 species) and computational mesh (8000 cells). For the same time step parameters, the solution time scales roughly as the mesh size, hence for the 18000 cell mesh the computational run time was about twice that of the 8000 cell mesh. Hence there are practical limitations on the grid size and number of species that can be included in the air chemistry model. Large simulation times also make the study of sensitivity to model parameters more difficult, which motivates the need for further algorithmic improvements for future studies in addition to the parallelization of continuum plasma models. 


\section{Chapter 4}

\section{Two-Dimensional Surface Plasma Actuator Study}

In this chapter, the air plasma chemistry model discussed in Chapter 3 is used to simulate bare electrode plasma actuator with plane electrodes (2-D). The effect of the bulk supersonic flow on the plasma discharge is simulated self-consistently using the formulation described in Chapter 2.

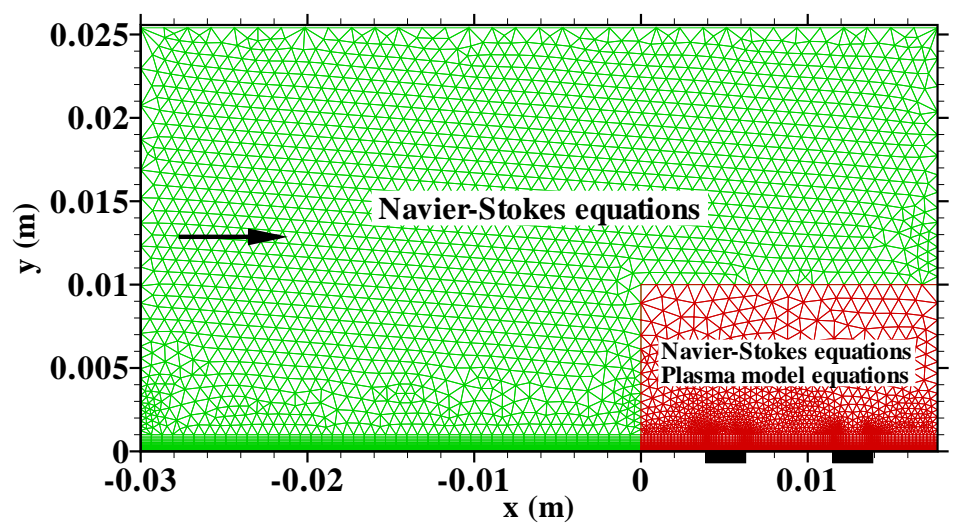

Figure 4.1: Computational domain and mesh for the plane plasma actuator simulations. The mesh contains 8504 cells, with the plasma model solved on the inset subdomain (in red) and the Navier-Stokes equations solved on the entire domain.

Figure 4.1 shows the actuator configuration used for the combined plasma-flow calculations. The electrodes are of length $2.4 \mathrm{~mm}$, and the distance between the centers of the electrodes is $7.62 \mathrm{~mm}$. The dimensions of 
the actuator are similar to those used in previous experiments [14]. However, the experiments were done with pin electrodes and so the discharge is $3-\mathrm{D}$, but the results shown in this chapter are two dimensional. The length of the computational domain is taken to be $\sim 5 \mathrm{~cm}$, representative of a typical flow length scale. The plasma discharge is confined to a region of a few $\mathrm{mm}$ above the surface, and hence the plasma model equations can be solved in an inset subdomain. The inset subdomain corresponds to the mesh region colored red in Fig. 4.1. Solving the air plasma model with 11 species on the entire domain is computationally prohibitive, and here we use the fact that surface discharge is localized to solve the plasma model on the inset subdomain alone. Figure 4.1 also shows the computational mesh used for the combined plasma-flow calculations. The unstructured mixed mesh has a total of 8504 cells, and the inset subdomain contains 4171 cells. Source terms are exchanged between the two models on the inset subdomain alone.

Figure 4.2 shows the inset subdomain in greater detail. The mesh is generated to provide higher resolution in the sheath region (and the supersonic boundary layer in the case of the flow solution), with a relatively coarse mesh in the farfield region. The highest electric fields in this configuration are typically found at the edge of the electrodes at the electrode-dielectric interface, and the mesh is refined to provide the highest resolution in that region.

The boundary conditions used for the plasma model in the present chapter are described below. The metal electrodes are exposed to the incoming supersonic flow, and the surrounding surface is modeled as a dielectric. For the species continuity equations, flux boundary conditions are applied 


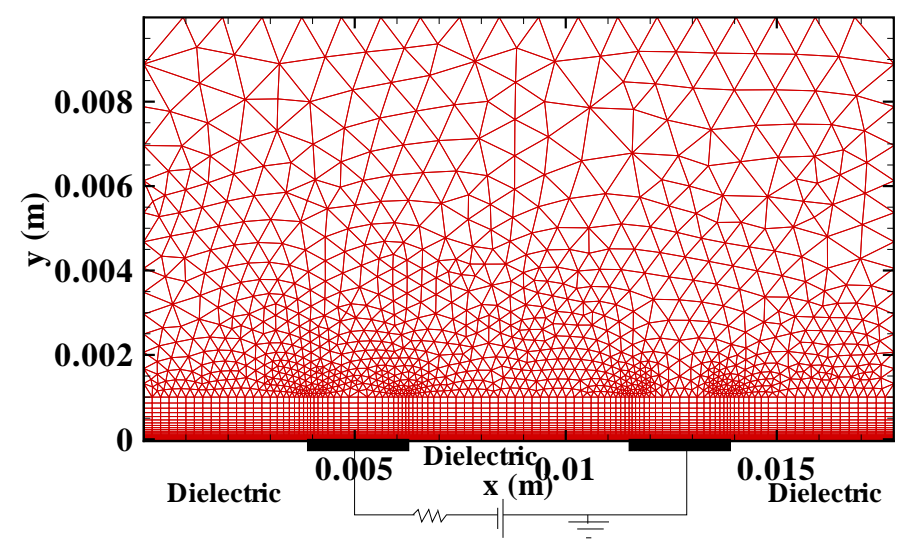

Figure 4.2: Computational mesh in the inset subdomain showing a schematic of the plane plasma actuator. The surface is modeled as a dielectric, with metal electrodes exposed to the incoming supersonic flow. The source voltage is applied at the cathode, with a resistance in series.

at solid surfaces (given by equations (2.8)-(2.10)). At the inflow to the inset subdomain, the species mole fractions of $\mathrm{N}_{2}$ and $\mathrm{O}_{2}$ are set to 0.79 and 0.21 respectively, and the rest of the species mole fractions are set to zero. The density is calculated from the pressure and the temperature given by the Navier-Stokes model at the cells adjacent to the boundary faces, and the mole fractions are used to specify the individual species densities. The fixed mole fraction boundary condition is also applied at the top boundary. On the right, the outflow boundary condition given by (2.12) is applied.

The potential at the dielectric boundaries are calculated using the thin dielectric boundary condition (2.14). The potential at the cathode is calculated using $V_{\text {cathode }}=V_{\text {source }}-i \mathrm{R}$, where $V_{\text {source }}$ is the source voltage, $i$ is the total current integrated over the cathode surface, and $\mathrm{R}$ is the specified series resistance. The potential at the anode is set to $0 \mathrm{~V}$ (ground). In the far-field, a 
potential boundary condition is applied such that no net current flows through the far-field boundaries. The thin dielectric boundary condition enforces the necessary zero current condition regardless of the distance at which the far field boundaries are located from the electrode surface. However, the thin dielectric boundary condition was found to add to the temporal stiffness of the model. The symmetry condition was instead found to provide zero current in the far-field, with essentially similar results for the plasma species densities and discharge currents while allowing a larger time step for the electron energy and density equations, and hence was used in the calculations presented here. Flux boundary conditions discussed in Section 2.1.2.3 are used for the electron energy equation at the surface, and symmetry boundary conditions are used at the farfield.

For the supersonic flow, the freestream conditions are $\mathrm{p}_{\infty}=2399 \mathrm{~Pa}(18$ Torr $), \mathrm{T}_{\infty}=110 \mathrm{~K}, \mathrm{U}_{\infty}=603.37 \mathrm{~m} / \mathrm{s}(\mathrm{M}=2.87)$, which are the same as those used in experiments [14]. At the wall surface, viscous wall boundary conditions (no-slip) with fixed wall temperature $=300 \mathrm{~K}$ are applied.

\subsection{Air surface plasma discharge in the absence of su- personic flow}

We first present results for the surface plasma discharge in stagnant air, with no bulk flow present. Figures 4.3-4.5 show charge density contours of the dominant negatively and positively charged species in the vicinity of the electrodes. The plasma model is solved on the inset subdomain shown in Fig. 4.2. The ambient pressure is fixed at 18 Torr. The cathode source voltage 


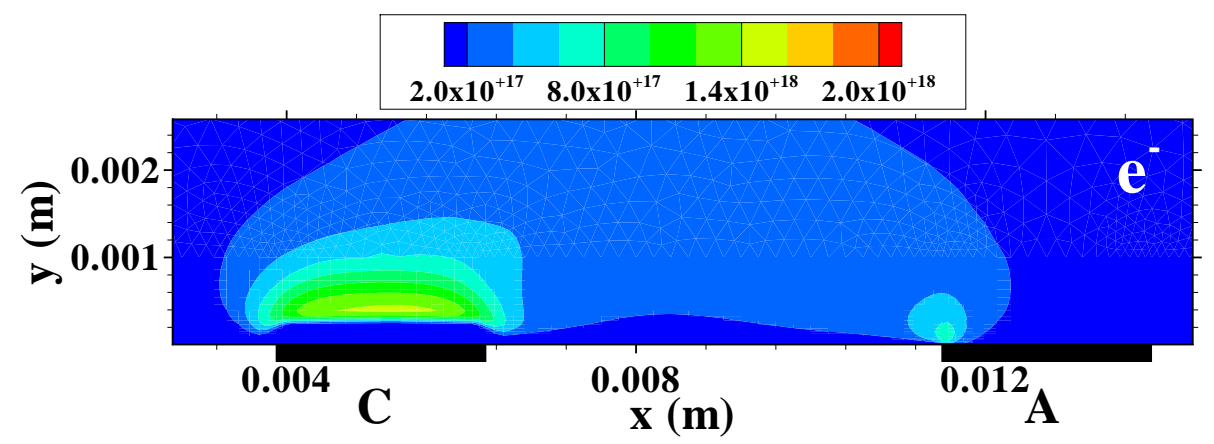

(a)

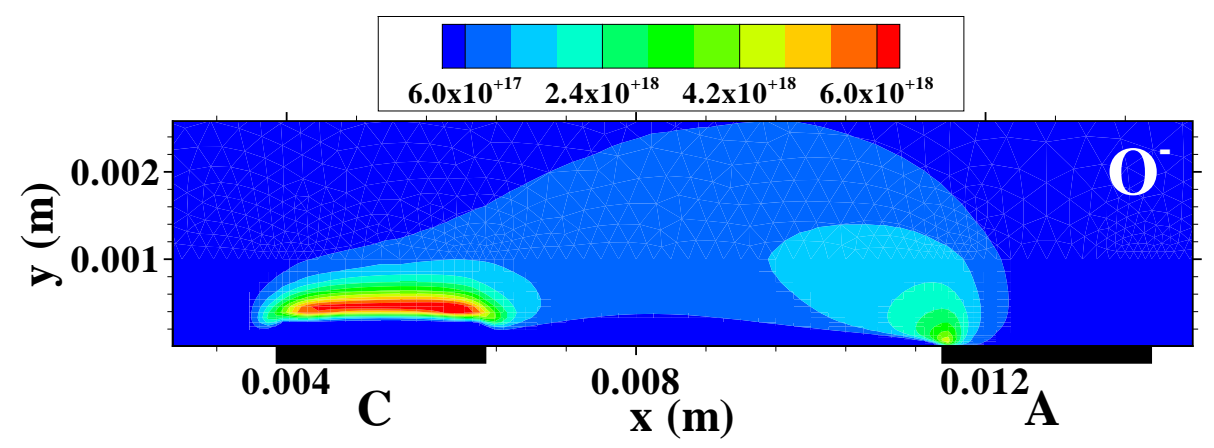

(b)

Figure 4.3: Species number densities (units: $\mathrm{m}^{-3}$ ) of (a) $\mathrm{e}^{-}$, and (b) $\mathrm{O}^{-}$ions in the surface plasma discharge (no bulk flow). The cathode source voltage is $-1000 \mathrm{~V}$, and the ambient pressure is fixed at 18 Torr.

is $-1000 \mathrm{~V}$, with a ballast resistance of $1 \mathrm{k} \Omega$. The electron density contours plotted in Fig. 4.3(a) show that the discharge is formed primarily above the cathode, with a spot attachment at the leading edge of the anode. Based on a depth of $1 \mathrm{~mm}$, the current is $\sim 10 \mathrm{~mA}$, which is similar to the low-power diffuse mode observed in experiments [14]. From Fig. 4.3(a) and Fig. 4.3(b) we see that the $\mathrm{O}^{-}$and electron densities are of comparable magnitude, and $\mathrm{O}^{-}$peak density is about three times that of the peak electron density. The peak $\mathrm{O}_{2}^{-}$density (not shown) is about 2 orders of magnitude smaller than $\mathrm{O}^{-}$, 


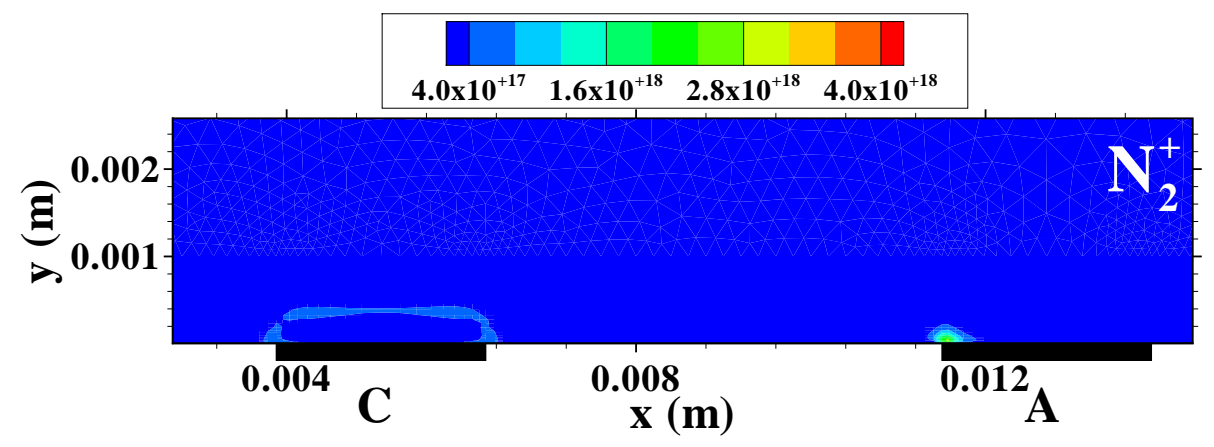

(a)

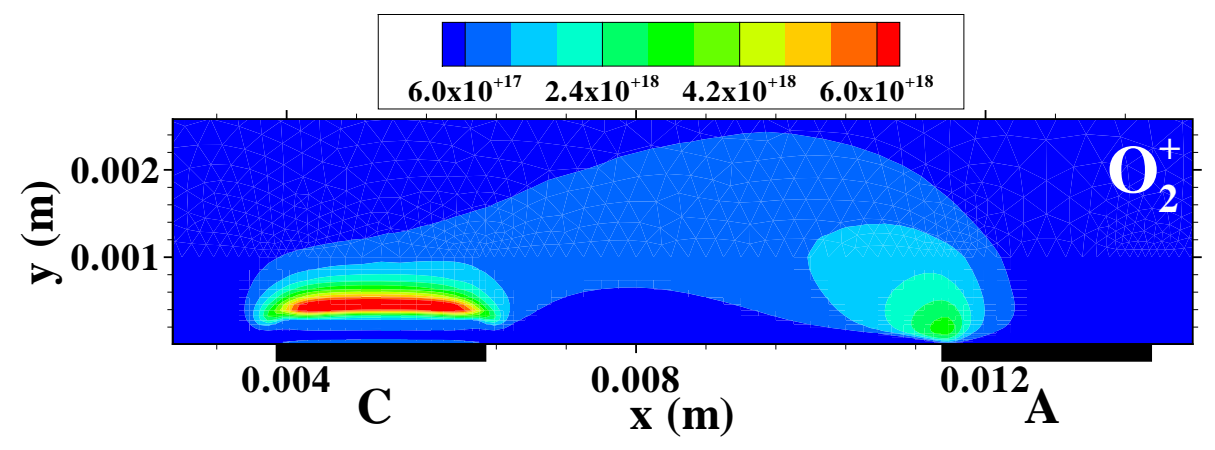

(b)

Figure 4.4: Species number densities (units: $\mathrm{m}^{-3}$ ) of (a) $\mathrm{N}_{2}^{+}$, and (b) $\mathrm{O}_{2}^{+}$ions in the surface plasma discharge (no bulk flow). The cathode source voltage is $-1000 \mathrm{~V}$, and the ambient pressure is fixed at 18 Torr.

which is in agreement with Fridman's estimate (pg. 34 in [73]) that threebody attachment to form $\mathrm{O}_{2}^{-}\left(\mathrm{G}_{12}\right.$ in Table 3.1) dominates over dissociative attachment that forms $\mathrm{O}^{-}$ions $\left(\mathrm{G}_{18}\right)$ at pressures $>30$ Torr.

Figures 4.4(a) and 4.4(b) show the density contours of $\mathrm{N}_{2}^{+}$and $\mathrm{O}_{2}^{+}$ions respectively. The dominant positive ion species in the bulk plasma is $\mathrm{O}_{2}^{+}$, and the dominant ion species in the cathode sheath is $\mathrm{N}_{2}^{+}$. As was the case in the air plasma validation study, the density of $\mathrm{O}_{2}^{+}$is higher than $\mathrm{N}_{2}^{+}$, which can be attributed to the higher ionization rate of $\mathrm{O}_{2}$ to form $\mathrm{O}_{2}^{+}$in the bulk plasma 


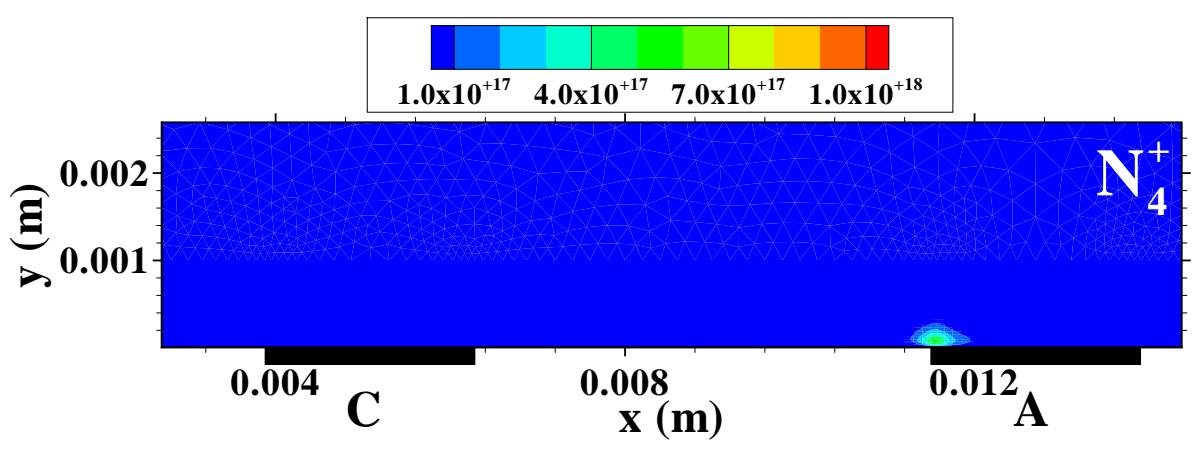

(a)

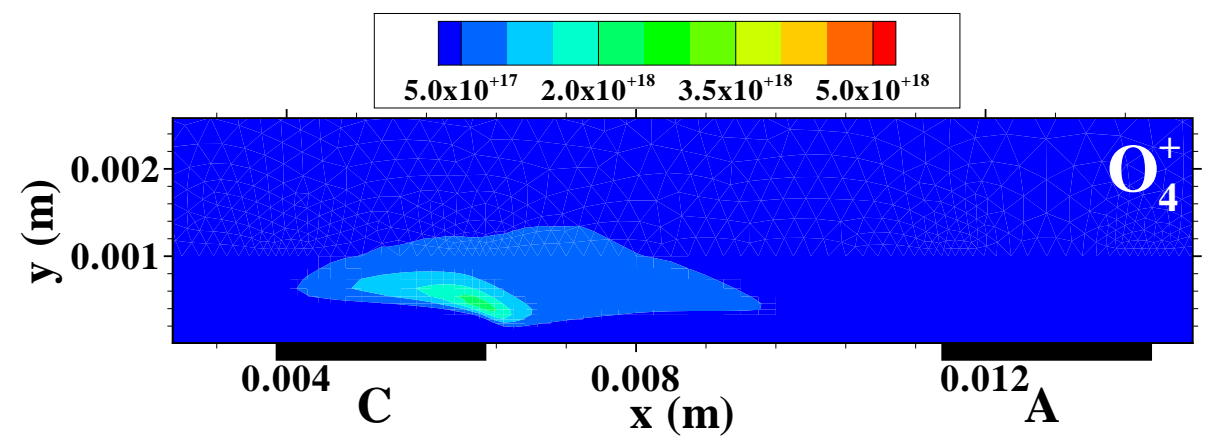

(b)

Figure 4.5: Species number densities (units: $\mathrm{m}^{-3}$ ) of (a) $\mathrm{N}_{4}^{+}$, and (b) $\mathrm{O}_{4}^{+}$ions in the surface plasma discharge (no bulk flow). The cathode source voltage is $-1000 \mathrm{~V}$, and the ambient pressure is fixed at 18 Torr.

(Table 3.1), and reaction $\mathrm{G}_{5}$ that converts $\mathrm{N}_{2}^{+}$to $\mathrm{O}_{2}^{+}$. At 18 Torr, the three body reaction rates are expected to be higher than in the validation case (600 mTorr), and peak the $\mathrm{N}_{4}^{+}$and $\mathrm{O}_{4}^{+}$densities shown in Figs. 4.5(a) and 4.5(b) are comparable to that of $\mathrm{O}_{2}^{+}$and $\mathrm{N}_{2}^{+}$in the case of the surface discharge in the absence of bulk flow.

In Table 3.1, $\mathrm{G}_{20}$ and $\mathrm{G}_{21}$ are used to model the energy lost by the electrons due to rotational, vibrational and electronic excitation reactions. In the case of a stationary discharge (no bulk flow), it is reasonable to assume that 


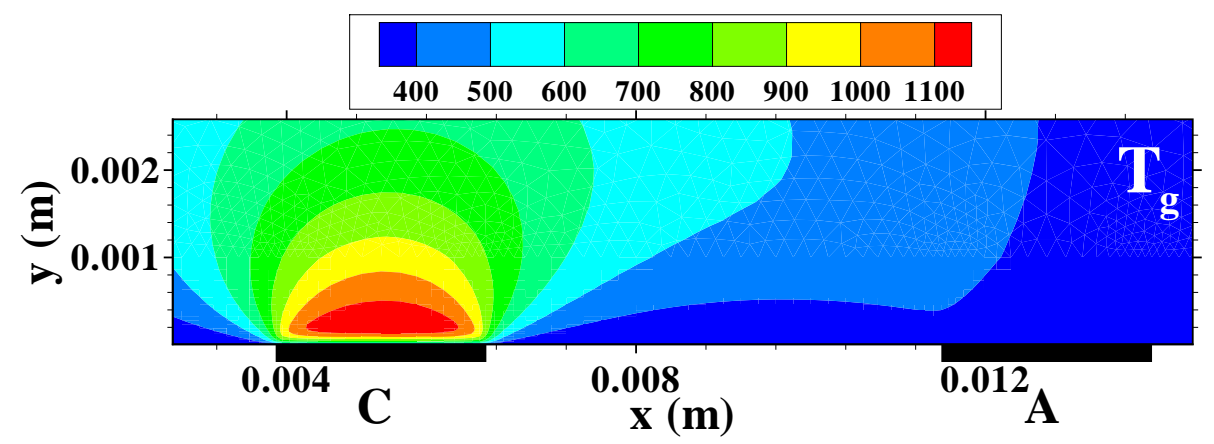

Figure 4.6: Gas Temperature contours (units: K) in the surface plasma discharge (no bulk flow). The cathode source voltage is $-1000 \mathrm{~V}$, and the ambient pressure is fixed at 18 Torr.

all the energy deposited into the excited states of the neutral gas is eventually transferred into the gas energy (translational) pool. Hence for the surface plasma actuator in the absence of flow we set $\Delta E^{g}=-1.0 \mathrm{eV}$ (adds energy to gas) for $\mathrm{G}_{20}$ and $\mathrm{G}_{21}$. The gas temperature contours are shown in Fig. 4.6. Dirichlet boundary conditions are used for all boundaries with the temperature fixed at $300 \mathrm{~K}$. An ion Joule heating thermalization factor $\eta_{\mathrm{Th}}=0.25$ was used to compute the ion Joule heating source term in (2.7). Significant gas heating is observed above the cathode with a peak gas temperature of $\sim 1180 \mathrm{~K}$, which is comparable to rotational temperatures of $1200-1800 \mathrm{~K}$ recorded in experiments [14] under similar discharge operating conditions.

\subsection{Air surface plasma discharge in the presence of su- personic flow}

We now present results of the plasma discharge solved in the presence of supersonic flow. In all cases presented in this section, the cathode is located 


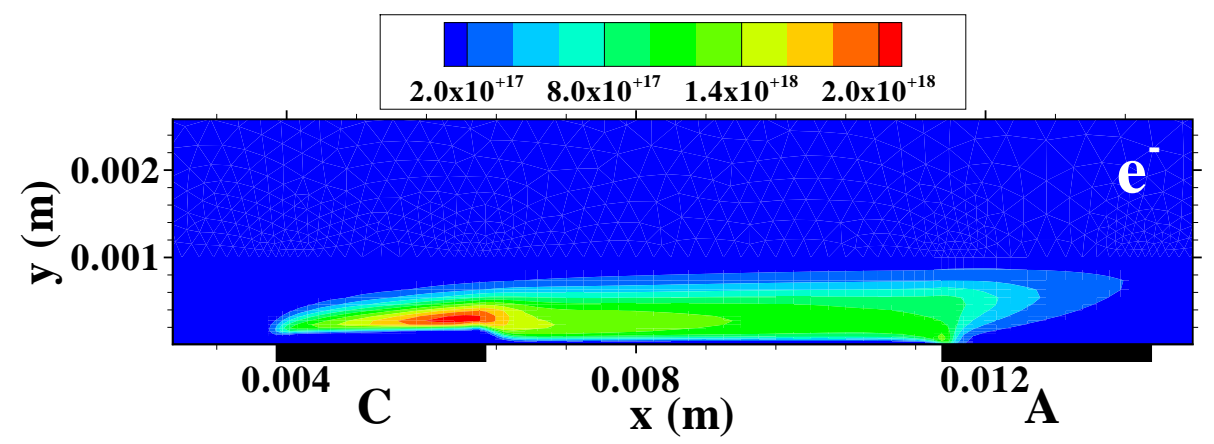

(a)

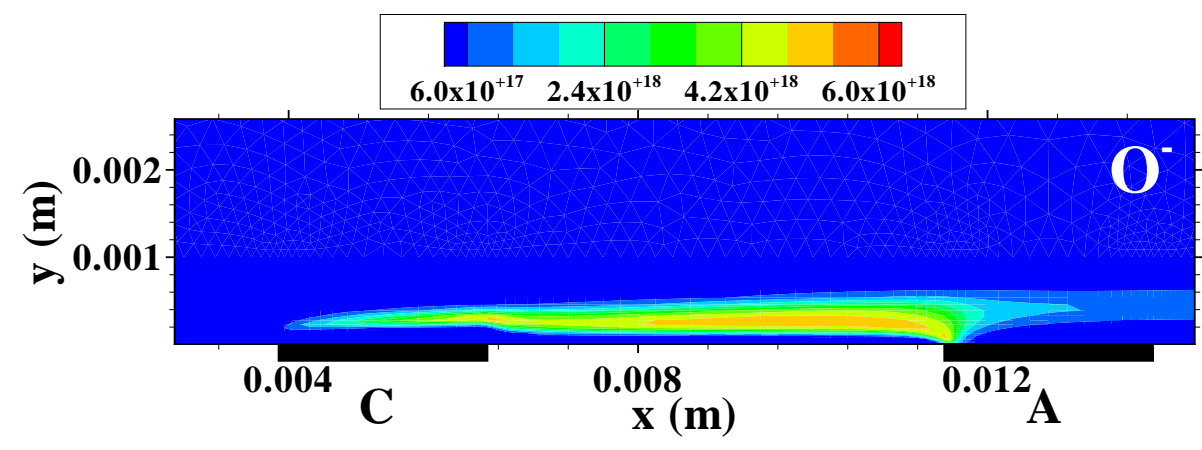

(b)

Figure 4.7: Species number densities (units: $\mathrm{m}^{-3}$ ) of (a) $\mathrm{e}^{-}$, and (b) $\mathrm{O}^{-}$ions in the presence of supersonic $M=3$ flow. The cathode source voltage is -1000 $\mathrm{V}$, and the freestream pressure is 18 Torr.

upstream with respect to the supersonic flow, and the convective effect of the supersonic flow on the plasma discharge is clearly seen with the charged species being swept downstream. The same cathode source voltage of -1000 $\mathrm{V}$ is used. Compared to the case of the plasma discharge with no supersonic flow, a higher current $\sim 12 \mathrm{~mA}$ is observed, accompanied by a corresponding increase in the electron number density, shown in Fig. 4.7(a). The electron density contours in Fig. 4.7(a) show that the peak electron electron density is located on the downstream edge of the cathode, with a small peak near the 


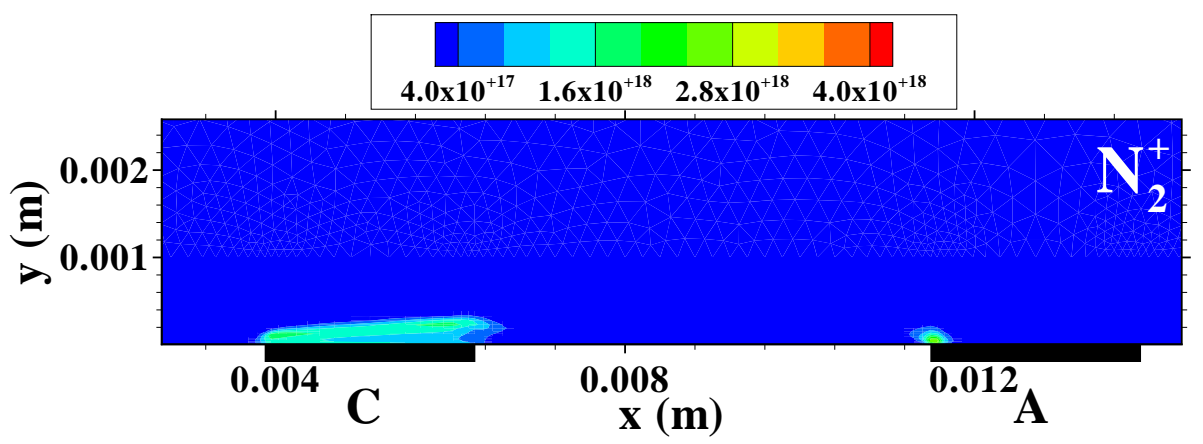

(a)

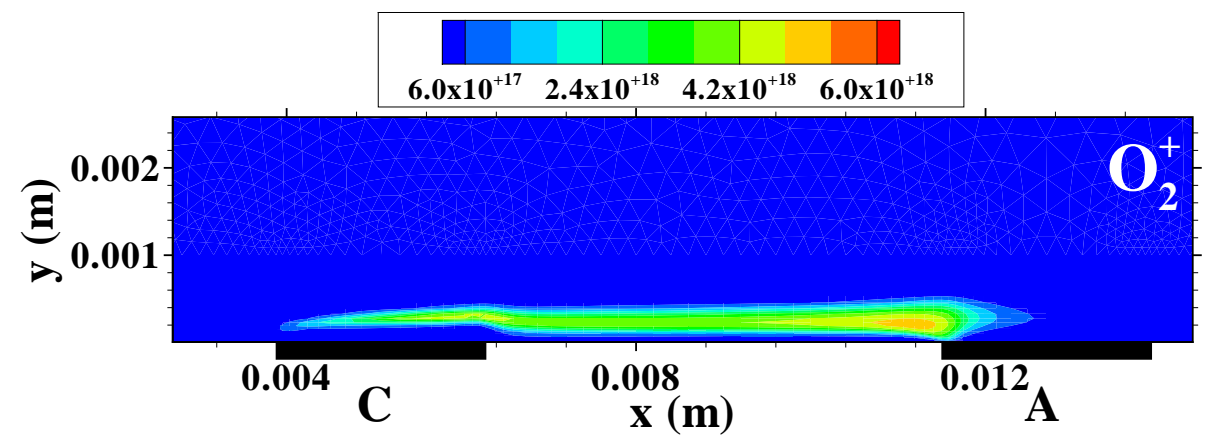

(b)

Figure 4.8: Species number densities (units: $\mathrm{m}^{-3}$ ) of (a) $\mathrm{N}_{2}^{+}$, and (b) $\mathrm{O}_{2}^{+}$ions in the presence of supersonic $M=3$ flow. The cathode source voltage is -1000 $\mathrm{V}$, and the freestream pressure is 18 Torr.

leading edge of the anode. In the region above the cathode the density of $\mathrm{O}^{-}$, shown in Fig. 4.7(b), is comparable to the electron density, and the peak $\mathrm{O}^{-}$ density is observed between the electrodes in the bulk plasma region. The charge density of $\mathrm{O}_{2}^{-}$(not shown) is two orders of magnitude lower than that of the electrons and $\mathrm{O}^{-}$.

Figures 4.8- 4.9 show the density contours of $\mathrm{N}_{2}^{+}, \mathrm{O}_{2}^{+}, \mathrm{N}_{4}^{+}$and $\mathrm{O}_{4}^{+}$ions in the presence of the supersonic flow. The ion drift velocities $\left(\sim 10^{3} \mathrm{~m} / \mathrm{s}\right)$ are of the order of the freestream flow velocity $(603.37 \mathrm{~m} / \mathrm{s})$. The charged 


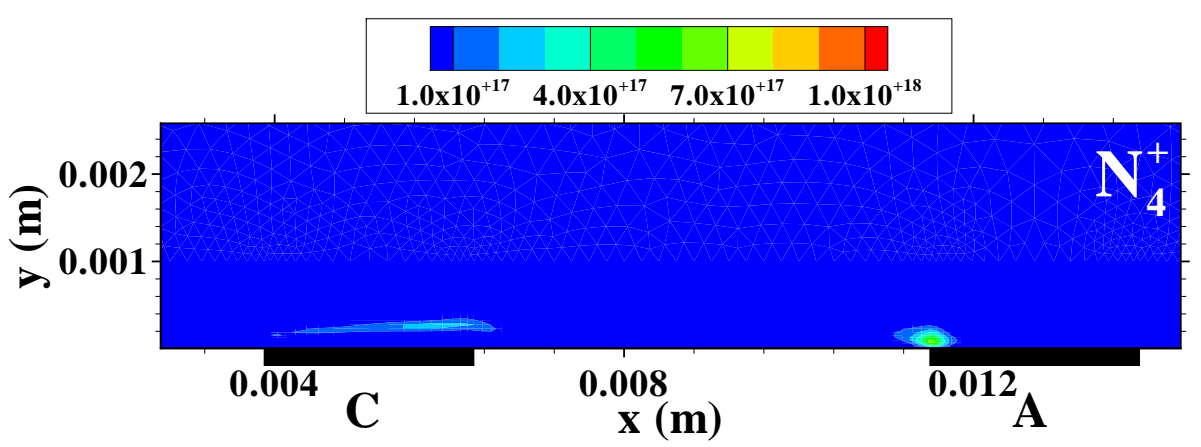

(a)

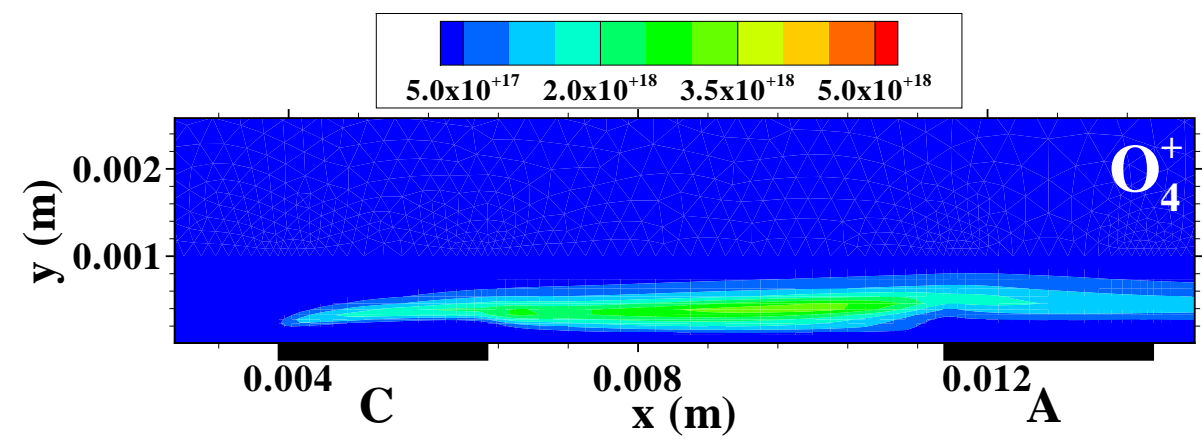

(b)

Figure 4.9: Species number densities (units: $\mathrm{m}^{-3}$ ) of (a) $\mathrm{N}_{4}^{+}$, and (b) $\mathrm{O}_{4}^{+}$ions in the presence of supersonic $M=3$ flow. The cathode source voltage is -1000 $\mathrm{V}$, and the freestream pressure is 18 Torr.

species are hence visibly affected by the bulk flow and are swept downstream compared to the no flow case shown in Figs. 4.3-4.5. The ambipolar fields cause the electron density to also be swept downstream in order to maintain quasi-neutrality in the bulk plasma. $\mathrm{N}_{2}^{+}$is the dominant positive ion present in the cathode sheath, with a second peak just above the leading edge of the anode.

While the dominant positive ion species in the bulk plasma without supersonic flow was $\mathrm{O}_{2}^{+}$, the dominant species in the presence of flow are 
$\mathrm{O}_{2}^{+}$and $\mathrm{O}_{4}^{+}$. As mentioned earlier, reaction $\mathrm{G}_{9}$ is one of the channels of $\mathrm{O}_{4}^{+}$production that has a strong gas temperature dependence (rate varies as $\left.T_{g}^{-3.2}\right)$. As we will see below, the supersonic flow has a cooling effect on the plasma by convecting the gas energy above the cathode away from the actuator region. From Figs. 4.8(b) and $4.9(\mathrm{~b}), \mathrm{O}_{4}^{+}$is found above the regions where $\mathrm{O}_{2}^{+}$ is significant. As we go away from the wall in the normal (y) direction, the gas temperature in the thermal boundary layer decreases, which results in an increased rate coefficient value from reaction $\mathrm{G}_{9}$. In addition, the decrease in gas temperature is accompanied by an increase in neutral density (downstream of the cathode, the pressure remains constant in the bulk plasma), so we can expect species production due to three-body reactions to increased neutral density. The combined effects of increased neutral density and increased rate coefficient due to reduced temperature result in increased $\mathrm{O}_{4}^{+}$production in the bulk plasma. $\mathrm{N}_{4}^{+}$density, shown in Fig. 4.9(a) is confined to a relatively thin region at the edge of the cathode sheath and the anode attachment region. As noted above, the bulk plasma is electronegative and an ion-ion core is formed in the bulk with $\mathrm{O}_{2}^{+}, \mathrm{O}_{4}^{+}$and $\mathrm{O}^{-}$ions. Downstream of the anode, in the afterglow region, $\mathrm{O}_{4}^{+}$and $\mathrm{O}^{-}$are the dominant positive and negatively charged species respectively.

For a plasma discharge in the presence of a flow, the lifetime of the metastable and vibrationally excited states compared to the flow time scales determines the fraction of energy that is transferred to the gas in the discharge region. The characteristic flow time scale for the current study based on an actuator length scale of $10 \mathrm{~mm}$ is of the order $10 \mu \mathrm{s}$. The vibrational lifetimes 


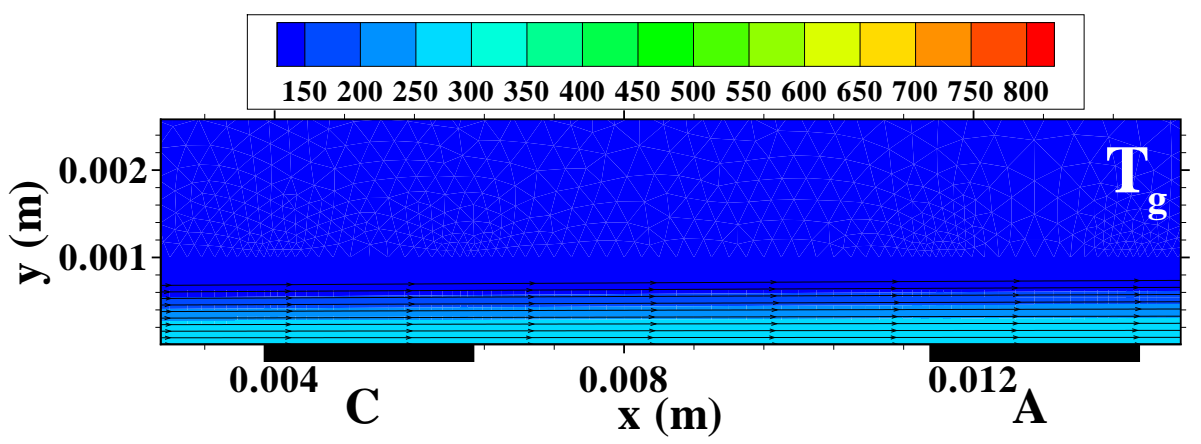

(a)

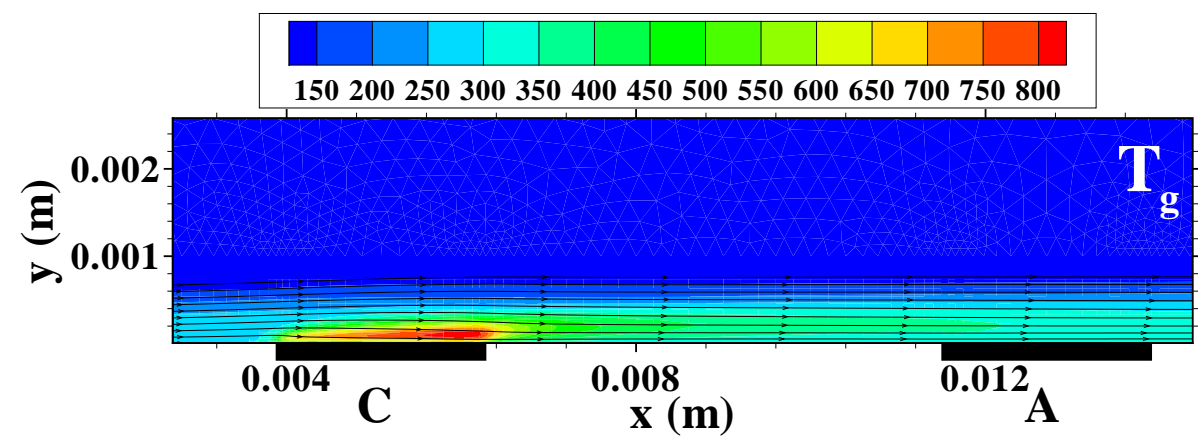

(b)

Figure 4.10: Gas temperature contours (units: K) in the supersonic flow with the plasma (a) off and (b) on. The wall temperature is fixed at $300 \mathrm{~K}$, and the freestream temperature is $110 \mathrm{~K}$. Velocity streamlines in the boundary layer are also shown.

of $\mathrm{N}_{2}$ and $\mathrm{O}_{2}$ in their parent gases are of the order of $10 \mathrm{~ms}([75]$, pg. 205). While the typical lifetime of metastables $\mathrm{N}_{2}\left(\mathrm{~A}^{3} \Sigma_{u}^{+}\right)$is of the order of a few seconds $\left([69]\right.$, pg. 58), they are collisionally quenched by $\mathrm{O}_{2}$, thereby contributing to gas heating. Therefore, we make the following assumptions: (1) energy gained by collisions resulting in vibrational excitation is lost due to convection, and (2) energy gained by collisions resulting in rotational excitation and generation of electronically excited states listed in Section 3.1 are immediately 
absorbed by the gas energy pool. The same ion Joule heating thermalization factor $\eta_{\mathrm{Th}}=0.25$ as in the no flow case was used to compute the ion Joule heating source term in (2.7) for the case with supersonic flow.

Figure 4.10 shows the gas temperature contours in the bulk flowfield in the absence and presence of the plasma discharge. The wall temperature is held fixed at $300 \mathrm{~K}$, and the freestream temperature is 110 K. From Fig. 4.10(a), we see that there is a thermal boundary layer present with a thickness of approximately $0.07 \mathrm{~mm}$. When the plasma is switched on, with the gas heating term calculated according to the assumptions listed in the previous paragraph, the peak gas temperature is found to be $\sim 830 \mathrm{~K}$, located above the downstream edge of the cathode. While the highest gas heating occurs in the cathode sheath, the supersonic flow convects the heat downstream of the cathode resulting in temperatures of $\sim 350-450 \mathrm{~K}$ in the bulk plasma.

Figure 4.11 shows the contributions of the components of the gas heating source term, i.e. the ion Joule heating, inelastic and elastic collisional heating. It is clear that the ion Joule heating is the dominant source of gas heating in the plasma, particularly in the cathode sheath where ions gain energy from the sheath electric fields and transfer the energy to the neutral gas through collisions. The inelastic heating source terms are composed of gas heating due to non-resonant charge exchange reactions (eg. $\mathrm{G}_{5}$ in Table 3.1), electron-ion and ion-ion recombination (eg. $\mathrm{G}_{11}$ and $\mathrm{G}_{19}$ ) and through relaxation of electronic and rotationally excited states of $\mathrm{N}_{2}$ and $\mathrm{O}_{2}$ listed in Section 3.1. The inelastic source terms are found to be comparable to the ion Joule heating in the bulk plasma and near the anode, while at the present 

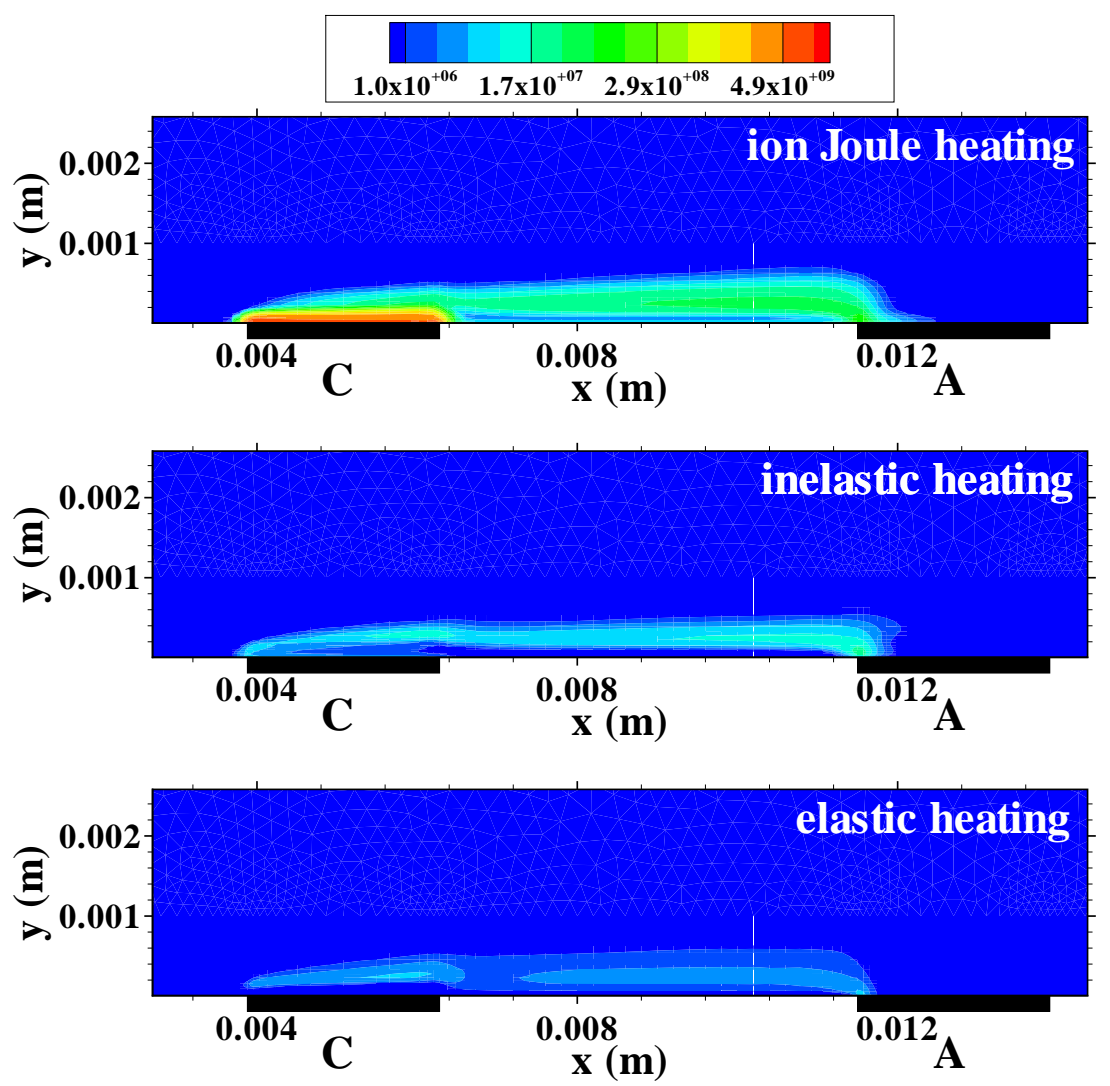

Figure 4.11: Gas heating source terms (units: $\mathrm{W} / \mathrm{m}^{3}$ ) in the plasma with supersonic flow, showing the contributions of ion Joule heating, inelastic and elastic heating sources to the overall gas heating.

operating pressure of 18 Torr, the contribution of the energy transferred to the gas by elastic collisions with electrons is negligibly small.

The effect of the gas heating on the pressure field is shown in Fig. 4.12. A weak shock is observed above the cathode, with a pressure rise of approximately $400 \mathrm{~Pa}$, shown in Fig. 4.12(b). The presence of the shock is primarily due to gas heating, and the electrostatic forcing in the sheath was found to cause no significant effect on the flow under the present discharge conditions. 


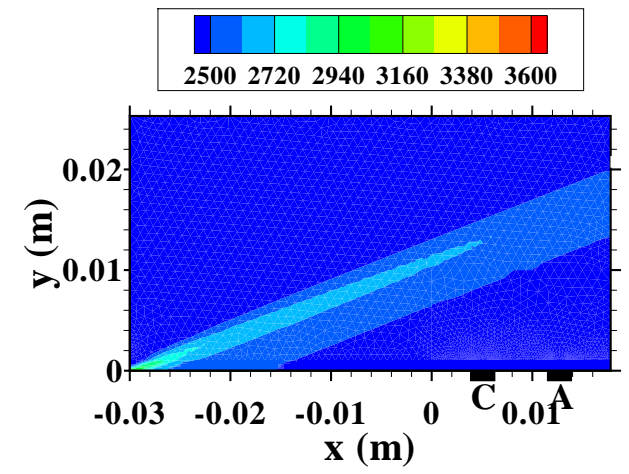

(a)

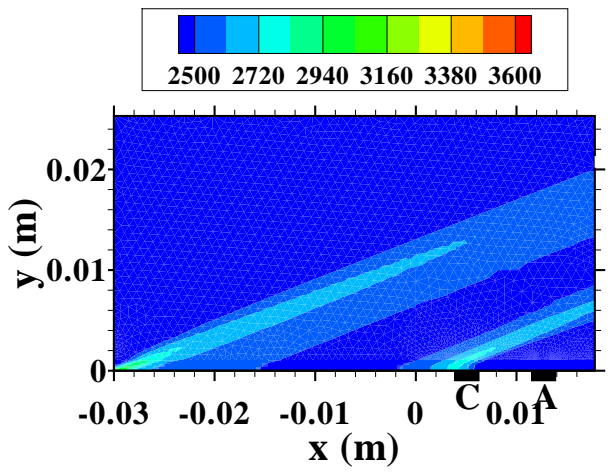

(b)

Figure 4.12: Pressure contours (units: $\mathrm{Pa}$ ) in the supersonic flow with the plasma (a) off and (b) on on the enitre flow domain.

Figure 4.13 shows the effect of varying the cathode source voltage $\mathrm{V}_{\text {source }}$ between $-800 \mathrm{~V}$ and $-1200 \mathrm{~V}$ at fixed freestream pressure 18 Torr. The cathode voltage $\mathrm{V}_{\text {cathode }}$ and current values for each case (based on a depth of $1 \mathrm{~mm}$ ) are indicated in Fig. 4.13(a), which shows the effect of varying the voltage on the electron number density. At the cathode source voltage of $-800 \mathrm{~V}$, the discharge appears to be in normal mode with only the downstream portion of the cathode covered by charged species. As the cathode source voltage is increased to $-1000 \mathrm{~V}$, the discharge covers the entire cathode area and further increase in voltage leads to higher electron densities and higher currents in the plasma. However, the discharge maintains a spot attachment at the upstream anode edge for all the three cases considered. The V-I curve corresponding to the cases shown in Fig. 4.13(a) is shown in Fig. 4.13(b). Hence the model is able to capture the transition from normal to abnormal mode glow discharge in the presence of supersonic flow. 


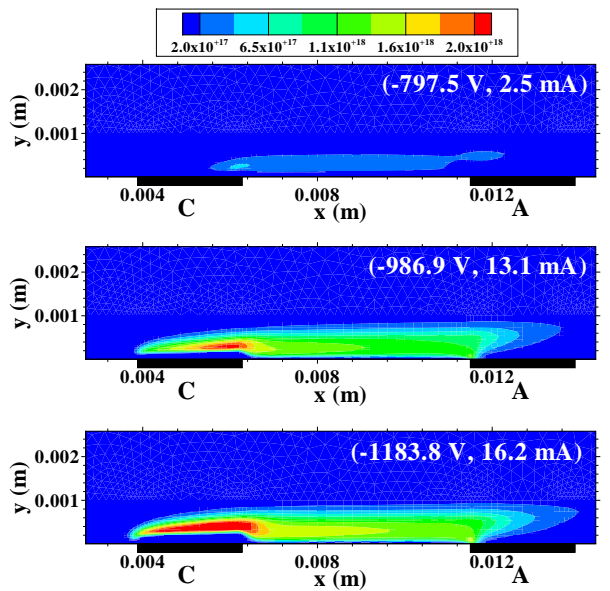

(a)

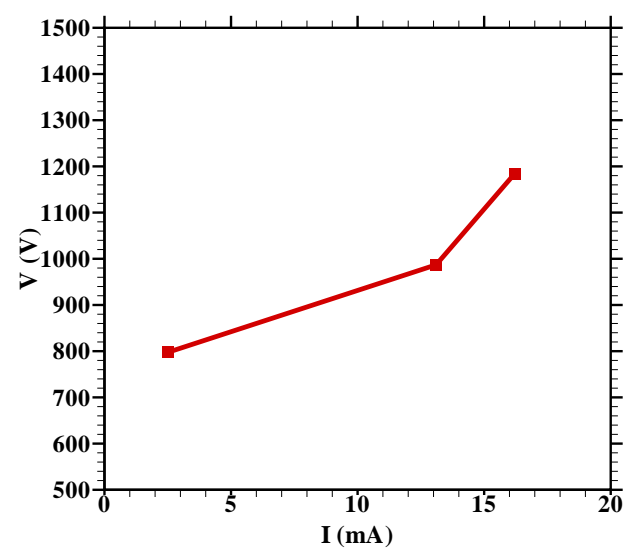

(b)

Figure 4.13: (a) Effect of voltage on the electron density contours at fixed pressure (18 Torr) and (b) corresponding V-I curve.

Figure 4.14 shows the effect of varying the operating freestream pressure between 16-20 Torr (2132.8-2666 Pa) with fixed cathode source voltage $\mathrm{V}_{\text {source }}=-1000 \mathrm{~V}$. All other flow parameters, i.e. freestream temperature, velocity and wall temperature are held fixed. Fig. 4.14(a) shows the effect of varying the freestream pressure on the electron number density. The electron density is found to increase with increasing pressure, however the magnitude of the increase is not very large. The discharge power input (based on depth of $1 \mathrm{~mm}$ ) is shown in Fig. 4.14(b) as a function of the pressure. We see that the discharge power increases by about $1.2 \mathrm{~W}$ as we go from 16 Torr to 20 Torr. This relatively small increase in power explains the relatively small increase in the electron number density as the freestream pressure is increased. Fig. 4.14(b) also shows the peak gas temperature in the discharge as a function of operating freestream pressure. It is observed that the peak gas temperature 


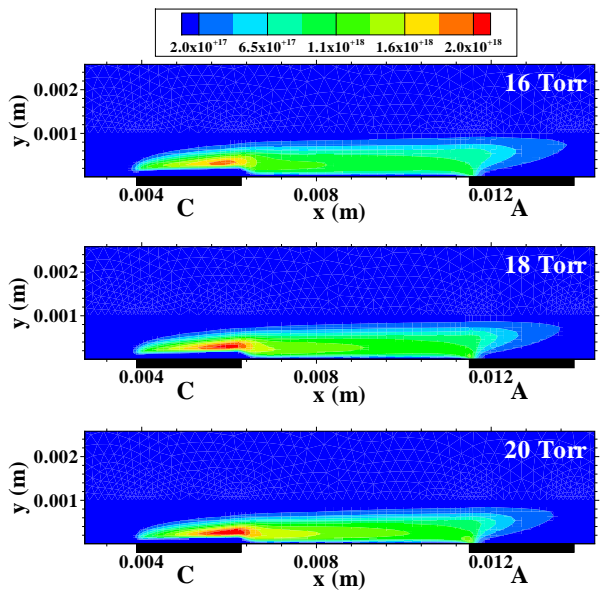

(a)

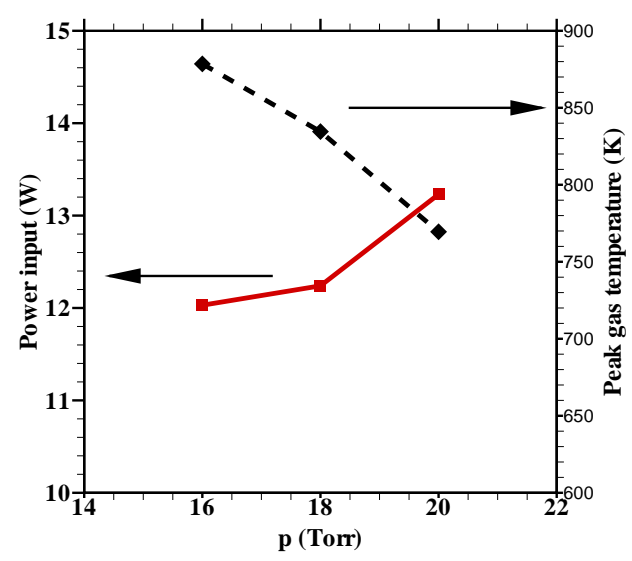

(b)

Figure 4.14: Effect of freestream pressure on (a) the electron density contours (units: $\mathrm{m}^{-3}$ ) and (b) discharge input power and peak gas temperature.

falls as the pressure is increased.

One possible explanation for the reduction of temperature is that for similar discharge input powers, the gas is heated more effectively when the background neutral density is lower. This is the case at $p=16$ Torr. As the pressure is increased, the thermal capacity of the gas increases due to increase in density, and consequently the peak gas temperature is smaller. The change in background density also influences the electrical conductivity, which could also explain the lower peak gas temperature observed when the freestream pressure is higher at similar discharge input power.

Figure 4.15 shows the sensitivity of the model results to the ion Joule heating thermalization factor $\eta_{\text {Th }}$. When the energy gained by ions in the sheath is completely thermalized $\left(\eta_{\mathrm{Th}}=1\right)$ the gas temperature is found to be 

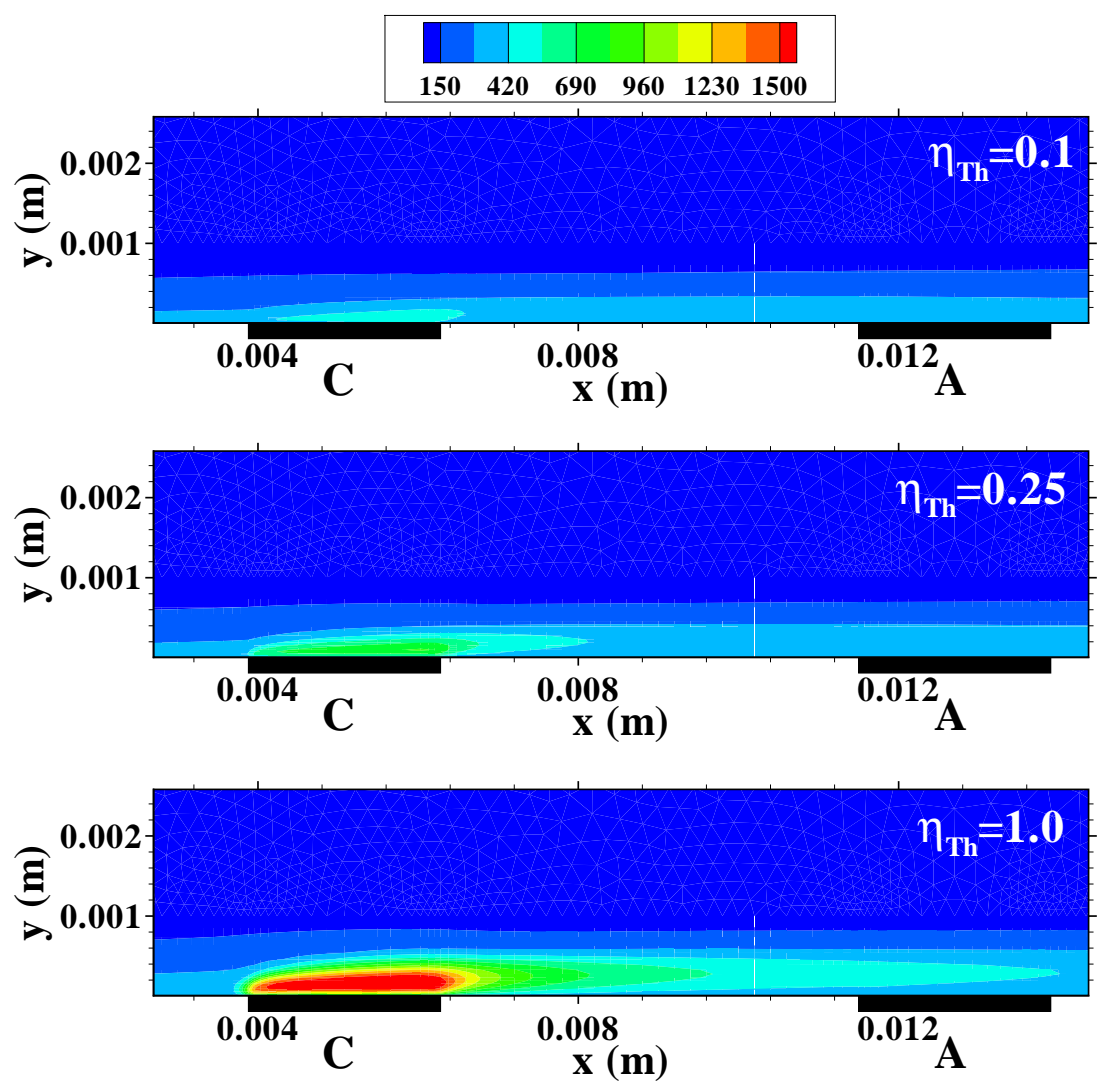

Figure 4.15: Sensitivity of gas temperature in the plasma with supersonic flow to the ion Joule heating thermalization factor $\eta_{\mathrm{Th}}$.

the highest, with a peak value of $1740 \mathrm{~K}$. The gas temperatures recorded in previous experiments under similar conditions [14] in the presence of supersonic flow are in the range of $600-900 \mathrm{~K}$. Hence the choice of $\eta_{\mathrm{Th}}=0.25$ used in the simulations above is a reasonable value. As $\eta_{\mathrm{Th}}$ is lowered further to 0.1 , the amount of gas heating is also lower leading to lower values of peak gas temperature $(540 \mathrm{~K})$. Although we have assumed $\eta_{\mathrm{Th}}$ to be a constant in the simulation domain, we note that the ion Joule heating, i.e. the dominant gas heating mechanism, mainly occurs in the cathode sheath, which is a relatively 
small region compared to the overall discharge volume. Hence, the assumption that $\eta_{\mathrm{Th}}$ is a constant is reasonable for the results presented in this chapter.

\subsection{Effect of electrode polarity}

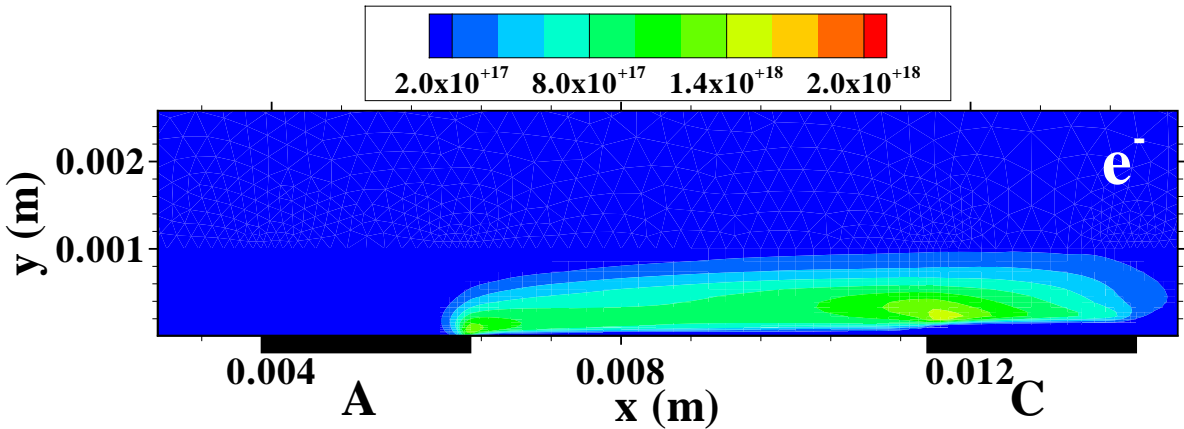

(a)

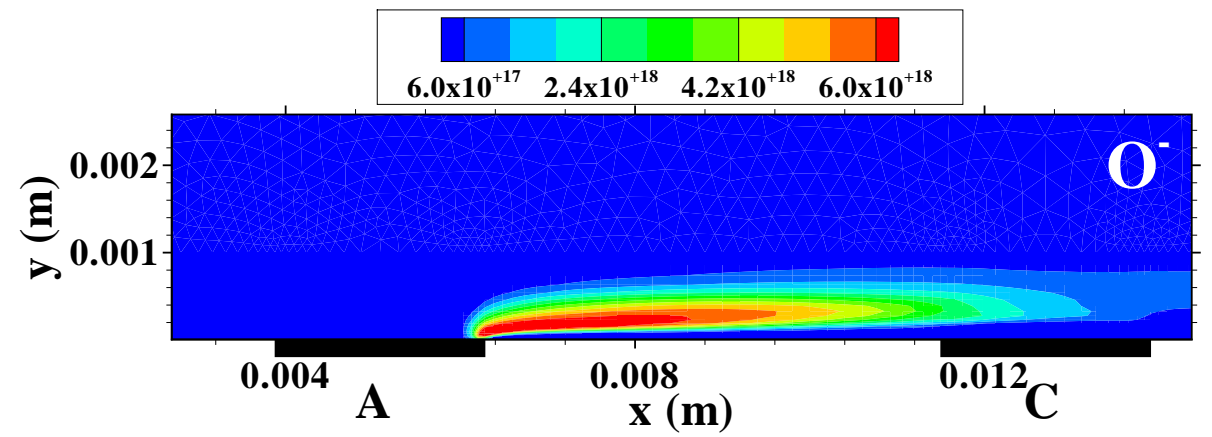

(b)

Figure 4.16: Species number densities (units: $\mathrm{m}^{-3}$ ) of $(\mathrm{a}) \mathrm{e}^{-}$, and (b) $\mathrm{O}^{-}$ions with the cathode located downstream with respect to the supersonic flow. The cathode source voltage is $-1000 \mathrm{~V}$, and the freestream pressure is 18 Torr.

In this section, the effect of the electrode polarity on the structure of the plasma and the gas heating is studied. The operating conditions are identical to the baseline case discussed in the previous section: cathode source voltage of $-1000 \mathrm{~V}$ with $1 \mathrm{k} \Omega$ series resistance, and freestream pressure 18 Torr. 


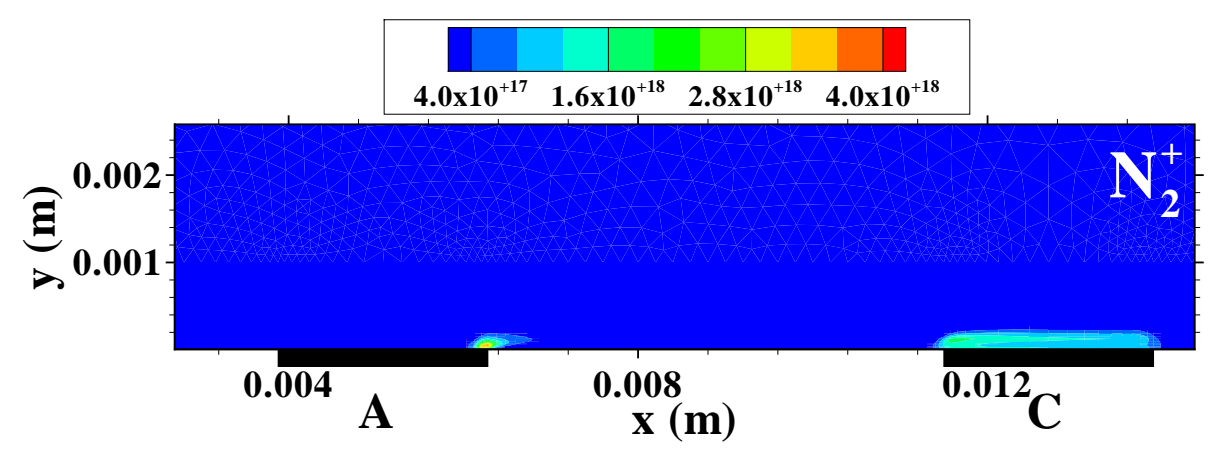

(a)

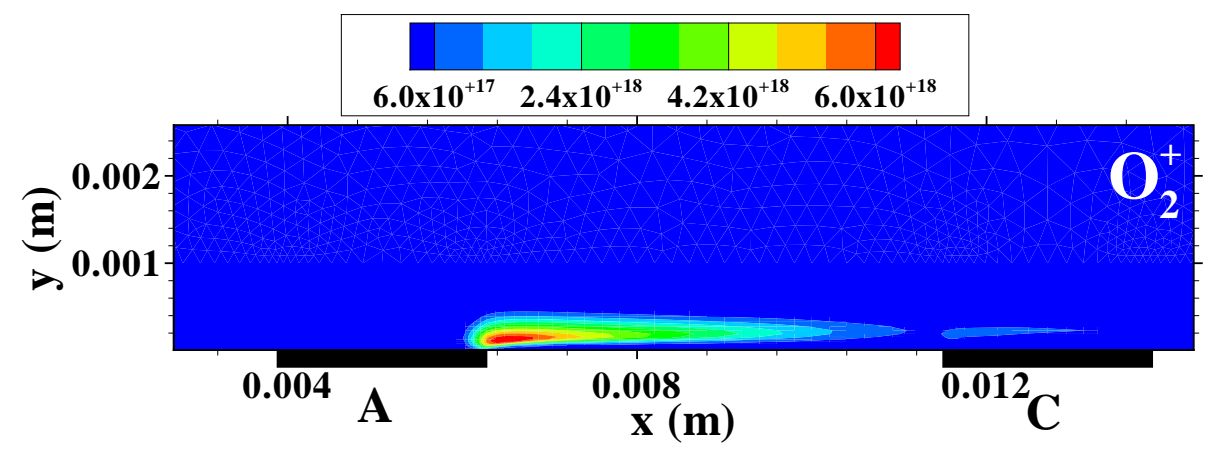

(b)

Figure 4.17: Species number densities (units: $\mathrm{m}^{-3}$ ) of (a) $\mathrm{N}_{2}^{+}$, and (b) $\mathrm{O}_{2}^{+}$ions with the cathode located downstream with respect to the supersonic flow. The cathode source voltage is $-1000 \mathrm{~V}$, and the freestream pressure is 18 Torr.

Figures 4.16-4.18 show charge density contours of the dominant charged species with the cathode located downstream with respect to the supersonic flow. The plasma discharge covers the cathode area with leading edge attachment at the anode. Based on a depth of $1 \mathrm{~mm}$, the current is $\sim 10.83 \mathrm{~mA}$. As was observed in the cathode upstream case, the charge density of $\mathrm{O}_{2}^{-}$(not shown) is two orders of magnitude lower than that of the electrons and $\mathrm{O}^{-}$. The electron density contours in Fig. 4.16(a) show that the peak electron density is located on the upstream edge of the cathode, with a small peak near the leading 


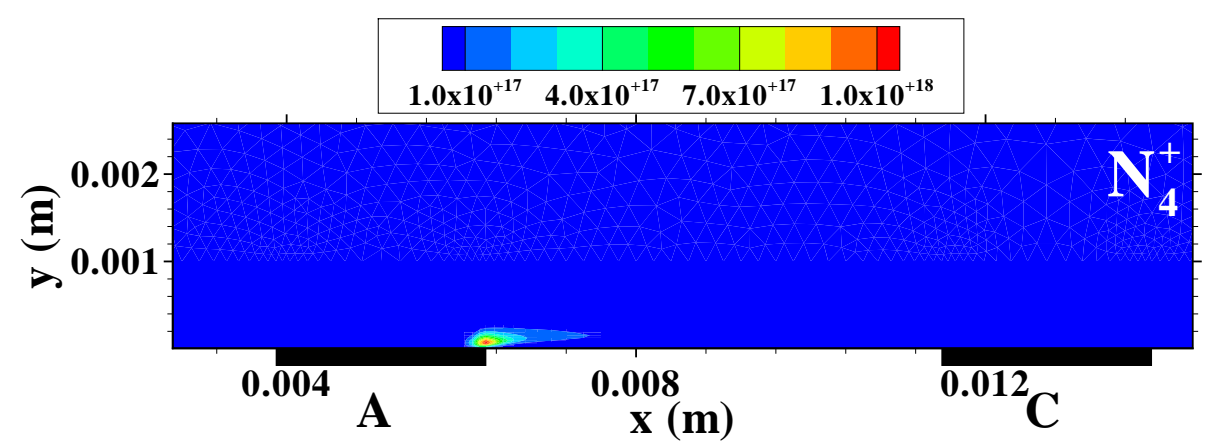

(a)

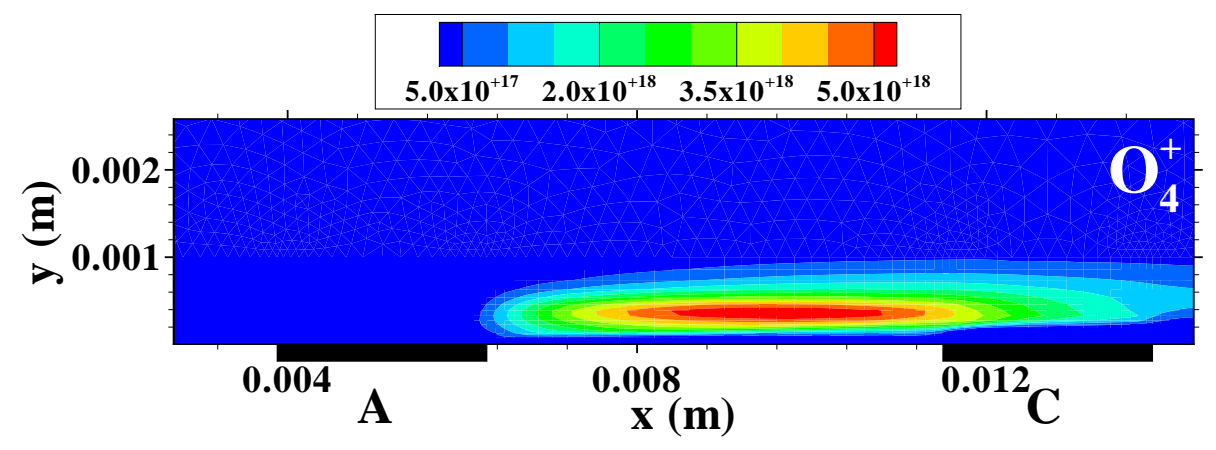

(b)

Figure 4.18: Species number densities (units: $\mathrm{m}^{-3}$ ) of (a) $\mathrm{N}_{4}^{+}$, and (b) $\mathrm{O}_{4}^{+}$ions with the cathode located downstream with respect to the supersonic flow. The cathode source voltage is $-1000 \mathrm{~V}$, and the freestream pressure is 18 Torr.

edge of the anode. The electron density looks qualitatively similar to that observed in the cathode upstream case with electron density extending above the cathode and a spot attachment to the anode. The $\mathrm{O}^{-}$charge density is shown in Fig. 4.16(b), from which we see that the peak density of $\mathrm{O}^{-}$is higher in the cathode downstream case to the cathode upstream case (Fig. 4.7(b)). Hence the cathode downstream discharge is more electronegative compared to the cathode upstream configuration.

As was observed in the cathode upstream case, $\mathrm{N}_{2}^{+}$is found in the 
cathode sheath with a prominent peak of $\mathrm{N}_{2}^{+}$density also observed in the vicinity of the anode. $\mathrm{N}_{4}^{+}$density, shown in Fig. 4.18(a), is confined to the anode attachment region. In the cathode upstream configuration, two peaks of $\mathrm{O}_{2}^{+}$density can be identified in Fig. 4.8(b): on the downstream side of the cathode where the electron density peak is observed in Fig. 4.7(a), and in the positive column where the peak $\mathrm{O}^{-}$density is found in Fig. 4.7(b). In contrast, the $\mathrm{O}_{2}^{+}$density map in the cathode downstream case shown in Fig. 4.17(b) has a single peak near the anode. From Fig. 4.18(b), $\mathrm{O}_{4}^{+}$is the dominant positive ion species, which indicates an increased three-body reaction rate from $\mathrm{G}_{9}$. From Figs. 4.18(b) and 4.16(b), we note the presence of an ion-ion core with $\mathrm{O}_{4}^{+}$and $\mathrm{O}^{-}$in the bulk plasma towards the anode, and in the afterglow region as well.

Figure 4.19 shows pressure and temperature contours when the cathode is located downstream with respect to the supersonic flow. As in the upstream case, when the plasma is turned on, volumetric heating of the supersonic flow by the plasma discharge takes place above the cathode surface in the boundary layer, resulting in a shock that is formed, as seen in Fig. 4.19(b). The shock wave in the cathode downstream case is weaker than the upstream case, with pressure rise of $\sim 300 \mathrm{~Pa}$. The cathode downsteam case has been shown to display weaker plasma actuation strength compared to the cathode upstream case in previous experiments $[14,15]$. The present model result is therefore consistent with the experimental results.

From Fig. 4.19(a) we observe a peak gas temperature of $\sim 620 \mathrm{~K}$ above 


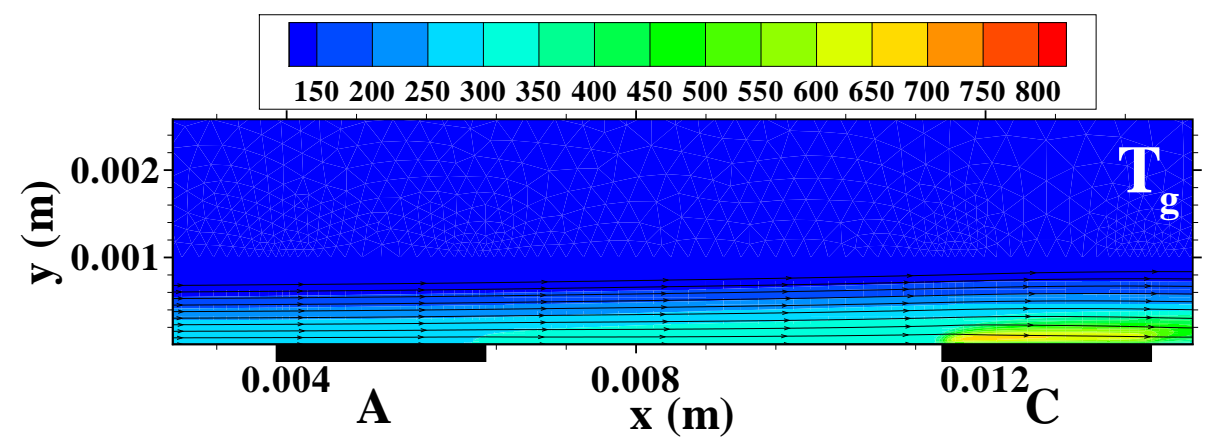

(a)

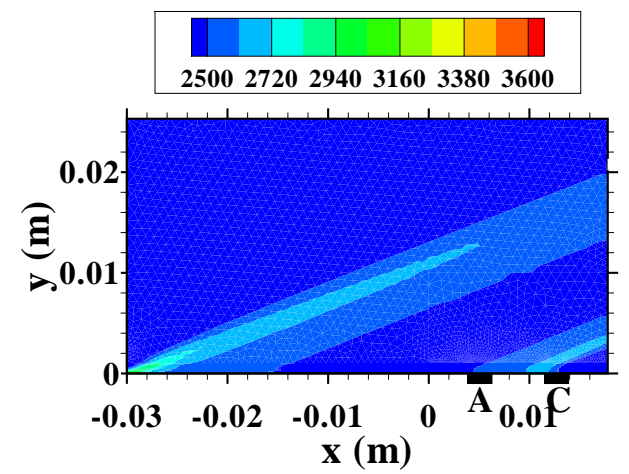

(b)

Figure 4.19: (a) Gas temperature (units: K) and (b) pressure contours (units: $\mathrm{Pa}$ ) in the supersonic flow with the cathode located downstream with respect to the supersonic flow.

the cathode surface, which is smaller than the peak temperature in the cathode upstream case but still comparable to rotational temperatures of $\sim 800 \mathrm{~K}$ measured in experiments [14] under similar discharge operating conditions. The gas temperatures in the bulk plasma region are of the order of $\sim 300-350$ K.

The differences in the observed charged species density profiles between cathode upstream and downstream case can be explained as follows. As men- 
tioned in the previous section, $\mathrm{G}_{9}$ has a strong gas temperature dependence (rate coefficient varies as $\mathrm{T}_{g}^{-3.2}$ ). The main effect of the plasma on the flow is gas heating above the cathode in both cathode upstream as well as cathode downstream configurations. In the cathode upstream case, the bulk plasma is formed downstream of the cathode where the thermal boundary layer has been altered by gas heating of the flow by the plasma. In the cathode downstream case, the bulk plasma is formed upstream of the cathode where the thermal boundary layer has not been significantly influenced by gas heating of the flow by the plasma. The altered thermal boundary layer profile in the bulk plasma region affects the chemistry, particularly three-body reactions that depend on the gas temperature.

We conclude by making some additional comments about the actuator model. The approach followed in this work, i.e. using separate models for the plasma and the bulk flow that can be self-consistently coupled together enables the simulation of cases where the surface plasma is solved with and without flow, and the flowfield is solved with the plasma model switched off. We observe that different ion species are dominant in the absence and presence of supersonic flow. The charged species densities also display a dependence on the electrode polarity with respect to the flow direction, which emphasizes the importance of including finite-rate chemistry in the plasma model. $\mathrm{O}^{-}$is the dominant negative ion species in all the cases studied in this chapter, and hence the plasma is electronegative, which is further indication that nitrogen cannot be used as a surrogate for air plasmas in the actuator simulations under the present conditions. 


\section{Chapter 5}

\section{Development of a Parallel Computational Tool for Plasma and Flow Simulations}

A parallel computing implementation of the plasma and flow simulation tools was developed as part of this research and is discussed in this chapter. The typical simulation time for results presented in the previous chapters with the serial implementation is about 3-4 weeks. The long simulation times are due to the highly disparate time scales (ranging from $10^{-11}-10^{-9} \mathrm{~s}$ for electrons to $10^{-5}-10^{-4} \mathrm{~s}$ for the ion diffusion and supersonic flow time scales), with corresponding long integration times to obtain a steady-state solution. To obtain solutions in a reasonable amount of time, there are practical limitations on the grid size ( 10,000 cells), and also in terms of number of species that can be incorporated in the chemistry model. Importantly, in practice the actuators have circular pin-like electrodes [14], making the problem three dimensional. It is not possible to simulate this configuration with the serial code. Therefore, there is a strong motivation to parallelize the models presented in Chapter 2. The serial algorithms are parallelized using the Message Passing Interface (MPI) library.

The plasma model discussed in Chapter 2 is implemented by solving

the governing equations in a segregated fashion, with the possibility of solving 
each equation on an independent set of physical subdomains which comprise the overall problem domain. For example, in Chapter 3, Poisson's equation is solved on all three physical subdomains, and the species conservation and electron energy equations are solved on the plasma subdomain alone (see Fig. 3.4). In Chapter 4, Poisson's equation, and the species conservation and electron energy equations are solved on the inset subdomain alone, while the compressible Navier-Stokes equations are solved on the entire problem domain (see Fig. 4.1). Hence the main requirement of the parallel algorithm is the ability to support multiple physical subdomains where different sets of equations may be solved. Certain equations, for example a circuit equation or the surface charge equation are solved on boundaries, and must also be solved in parallel.

\subsection{Implementation}

The three main aspects of the parallel implementation: mesh partitioning, communication of data between processors, and solution of the discretized equations are described below.

METIS [76] is a popular choice for domain decomposition and is used in the present work. The input to METIS is a graph of the mesh on which the problem of interest is being solved. The models described in Chapter 2 are discretized using a cell-centered finite volume scheme with general mixed meshes with support for triangle and quadrilateral cells in 2-D, and tetrahedral, pyramid, prism and hexahedral cells in 3-D. In the cell-centered scheme, the mesh cell centroids form the "nodes" of the graph, and the mesh faces between 
two cells form the "edges" of the graph (independent of the cell type). In the current parallel implementation, each processor reads the entire mesh file and determines the equivalent graph in compressed row storage (CSR) format. The METIS library functions are called on each processor, producing identical partitions on each of the processors. The partition mesh that corresponds to the current processor rank is retained, and the remaining parts of the mesh (cells, faces, nodes, boundary and connectivity information) are deleted.

In this work we use the term "subdomain" to refer to a physical subdivision of the mesh, while the term "partition" is used to refer to a computational sub-division of the mesh. A "physical boundary section" is a surface on the boundary of the overall problem domain, or a surface that lies between physical subdomains. Boundary/interface conditions need to be enforced at physical boundary sections. A "partition boundary section" is a surface that lies between partitions in the mesh. Variables need to be communicated across partition boundary sections.

METIS supports multi-constrained partitioning with the ability to specify weights for edges and nodes in the graph, both of which are very important in the context of the plasma and fluid models solved on multiple physical subdomains. The edgecut of the partitioning is defined as the number of graph edges (faces in the input mesh) that extend across partitions. If the graph edges are weighted, then the edgecut is defined as the sum of the weights of graph edges that extend across partitions. The default objective function for the partitioning is to minimize the edgecut of the input graph. From a practical viewpoint, it is easier to implement the algorithm if we assume faces 
that lie on physical boundaries between subdomains (in the global mesh) do not fall on partition boundary sections. The above requirement can be enforced by setting a large weight on the faces (graph edges) that lie on physical boundaries between subdomains. To account for the fact that mesh cells in certain physical subdomains can experience heavier load since a higher number of equations may be solved on them, cell weighting (node weighting in the input graph) can also be done. In Section 5.2, the effect of cell weighting on the algorithm scaling will be examined.

Communication between processors is performed using standard nonblocking MPI library functions. At each partition boundary section of a given processor rank, the variables to be communicated are collected into a single buffer and sent to the neighboring processor rank. At the same time, variables from the neighbor rank are received in a buffer, unpacked and stored. The received variables are "ghost variables", and are treated similar to the interior variables. To minimize the latency associated with the process and improve program scalability, communication is overlapped with calculation wherever possible. In the serial algorithm, the fluxes and matrix coefficients required to solve the discretized equations of the form given in (2.19) are typically calculated by looping over interior and physical boundary faces, as shown in Fig. 5.1.

In Fig. 5.1, the unshaded boxes represent the serial implementation, and the shaded boxes represent the additions to the serial code. As seen from the figure, the parallel algorithm is implemented in a way that augments the 


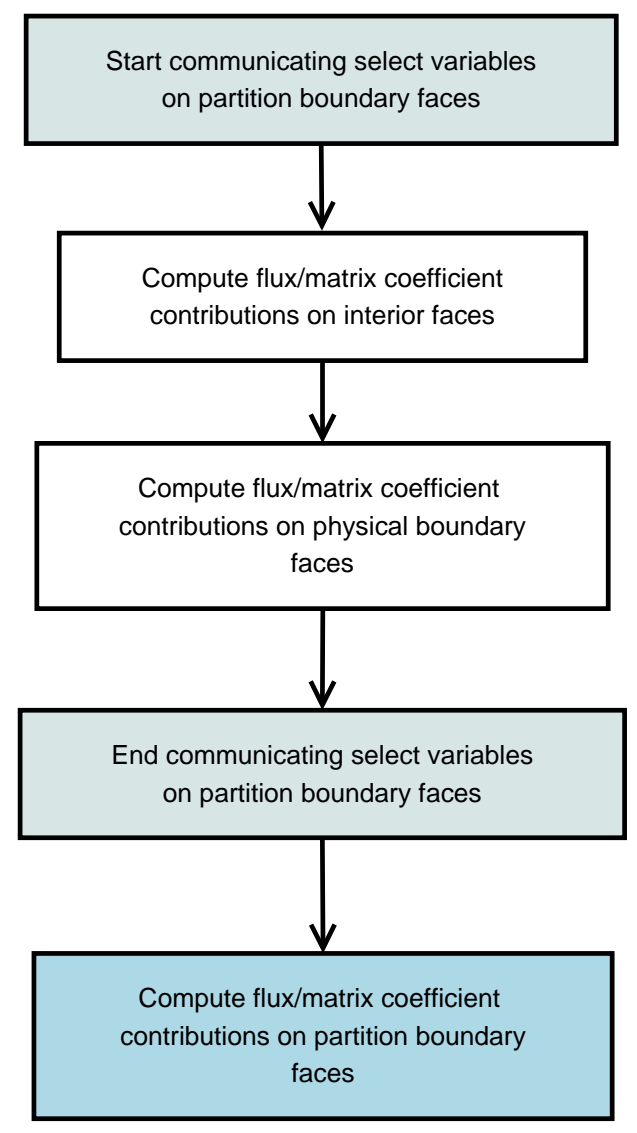

Figure 5.1: Parallel algorithm to calculate fluxes at faces and matrix contributions at cells adjacent to faces. The unshaded boxes represent the serial implementation, and the shaded boxes denote additions to the serial implementation.

serial code while minimizing changes in the existing code.

The discretized species continuity, potential, electron and gas energy equations in the serial implementation are solved using PETSc [37]. Given that the serial algorithm solves the equations sequentially in a segregated manner, each discretized equation is cast in the form $A \mathbf{x}=\mathbf{b}$ and solved using PETSc's Krylov Subspace (KSP) methods. In the parallel implementa- 
tion, the first step is to define MPI sub-communicators for each equation that are a subset of MPI_COMM_WORLD. MPI sub-communicators are created corresponding to subdomains where a given equation is solved, and a PETSc solver context is created for that particular sub-communicator. Once the matrix coefficients and fluxes are calculated on each partition, the matrices and vectors are assembled in parallel and the linear system is solved on the processors that belong to the corresponding MPI sub-communicator. When the flow model is to be solved along with the plasma model, the flow equations are solved in parallel by defining the appropriate MPI sub-communicator depending on the subdomains where the flow is to be computed.

MPI sub-communicators are also created for all physical boundary sections of the input mesh. The external electrical circuit equation solved in this work is of the form $\mathrm{V}_{b}=\mathrm{V}_{s}-i \mathrm{R}$, where $\mathrm{V}_{s}$ is the source potential (given), $\mathrm{R}$ is a series resistance (given), $\mathrm{V}_{b}$ is the potential at the connection boundary that needs to be found, and $i$ is the total current flowing across the connection boundary. Using the functions present in the serial implementation, partial currents are calculated on processors that contain part of the connection boundary. The total current $i$ is calculated from the partial currents using MPI collective communication (MPI_Allreduce), and the potential at the boundary $\mathrm{V}_{b}$ is found. 


\subsection{Verification of the parallelized models and scalabil- ity}

In this section, the parallel implementation is verified by comparing to serial model results. Two verification cases are presented in this section: a Gaseous Electronics Conference RF reference cell [77], which has a single physical subdomain, and the air chemistry validation presented in Chapter 3 with multiple physical subdomains.

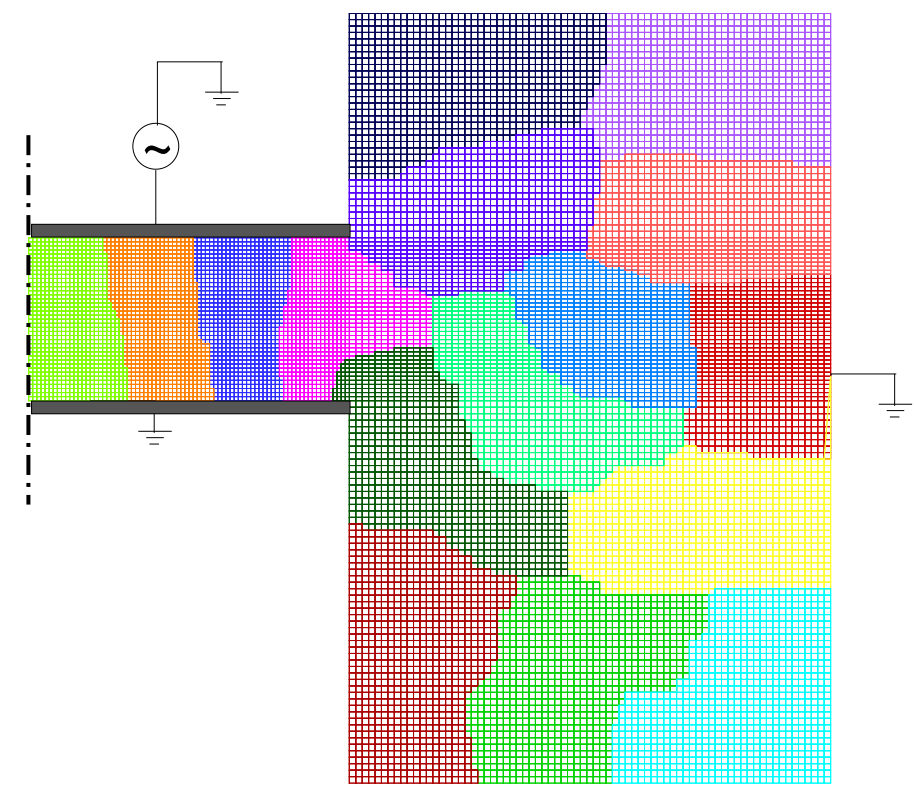

Figure 5.2: Schematic and mesh of the GEC RF reference cell. The mesh shown has 13170 cells, and is divided into 16 partitions using METIS.

The first problem chosen for parallel verification is a Gaseous Electronics Conference (GEC) RF reference cell [77], a schematic of which is shown 
in Fig. 5.2. The mesh shown in Fig. 5.2 has 13,170 cells divided into 16 partitions using METIS [76]. The top electrode is powered by a $200 \mathrm{~V}$ peakto-peak $13.56 \mathrm{MHz}$ voltage source, and the other electrode is set to ground potential $(0 \mathrm{~V})$. The walls of the reference cell are also set to ground potential. The pressure is fixed at 100 mTorr, and the gas temperature is fixed at $300 \mathrm{~K}$. An argon plasma with 4 species and 6 reactions (argon chemistry sub-mechanism from [78]) is used for the simulations. The potential, species density and electron energy equations are solved using the GMRES algorithm with Block Jacobi preconditioning, and Incomplete LU factorization (ILU(0)) on each block (see documentation from [37] for more details).

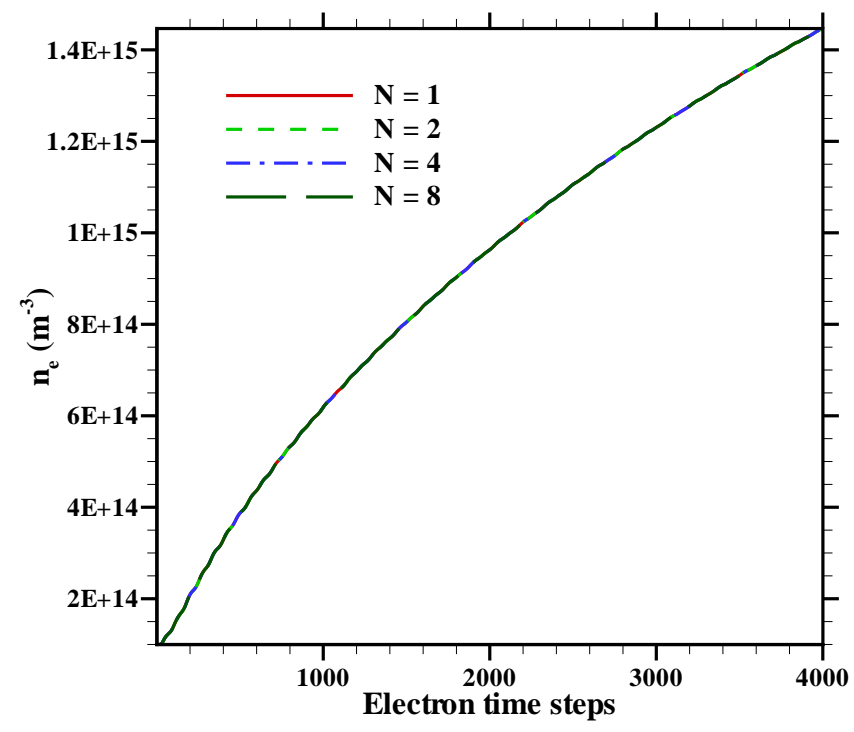

Figure 5.3: Plot of the electron number density (units: $\mathrm{m}^{-3}$ ) on the axis at the midpoint between the electrodes as a function of the number of electron time steps on $\mathrm{N}=1,2,4$ and 8 processors.

Figure 5.3 shows the evolution of the electron number density as a 
function of the electron time steps at a location on the axis and at the midpoint between the electrodes shown in Fig. 5.2. Each case was run for 4000 electron time steps, for number of processors $\mathrm{N}=1,2,4$ and 8. As seen from Fig. 5.3, the results for $\mathrm{N}=2,4$ and 8 are virtually identical to the serial $(\mathrm{N}=1)$ case.

Figure 5.4 shows the strong scaling curve for the GEC RF reference cell with a fixed mesh size of 13170 cells on 1-16 processors. Each case was run for 4000 electron time steps, and the runs were made on the Ranger supercomputer at the Texas Advanced Computing Center [79]. Strong scaling refers to the study of the parallel performance given a fixed total problem size. The speedup $\Psi=t_{1} / t_{N}$ is defined as the ratio of the time taken to solve the problem on 1 processor to the time taken to solve the problem on $\mathrm{N}$ processors, and is used as a measure of parallel performance. We note that for strong scaling, $\Psi(\mathrm{N})=$ $\mathrm{N}$ in the ideal case. From Fig. 5.4 we see that a maximum calculated speedup of $\sim 13$ is obtained for $\mathrm{N}=16$ processors. For the mesh size of 13,170 cells, the performance does not improve beyond 16 processors on Ranger.

The second parallel verification case considered is the air plasma validation discussed in Chapter 3. The air plasma validation case has three physical subdomains, shown in Fig. 3.4: a plasma subdomain bounded by a quartz ring, and an outer subdomain modeled as a dielectric. Figure 5.5 shows the evolution of the cathode current as a function of the electron time step, for the serial case $(\mathrm{N}=1)$ and a parallel case with $\mathrm{N}=8$ processors. As was observed in the GEC RF reference cell test case, identical results are observed in Fig. 5.5 for the serial and parallel cases. 


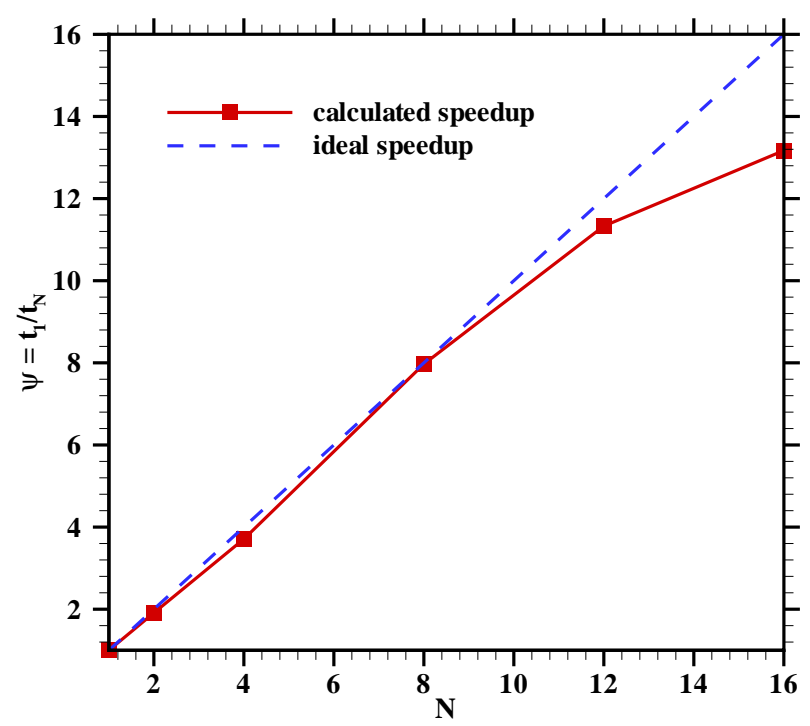

Figure 5.4: Strong scaling curve for the GEC RF reference cell with 13,170 cells on 1-16 processors.

Figure 5.6 shows the air plasma validation mesh with 22,920 cells divided into 8 partitions using two methods. In Fig. 5.6(a), all cells in the mesh are given equal weight $(=1)$ in the METIS partitioning, resulting in 8 equisized partitions each containing 2865 cells. In Fig. 5.6(b), cells that are in the plasma subdomain are assigned a weight of 12 , and cells in the quartz and outer subdomains are assigned a weight of 1 . The choice of weights is motivated by the number of governing equations solved in the physical subdomains. In the plasma subdomain, Poisson's equation, 10 species continuity equations, and the electron energy equation are solved. In the quartz and outer subdomains, only Poisson's equation is solved for the electrostatic potential. The partitions that result from METIS given the chosen cell weighting scheme are 


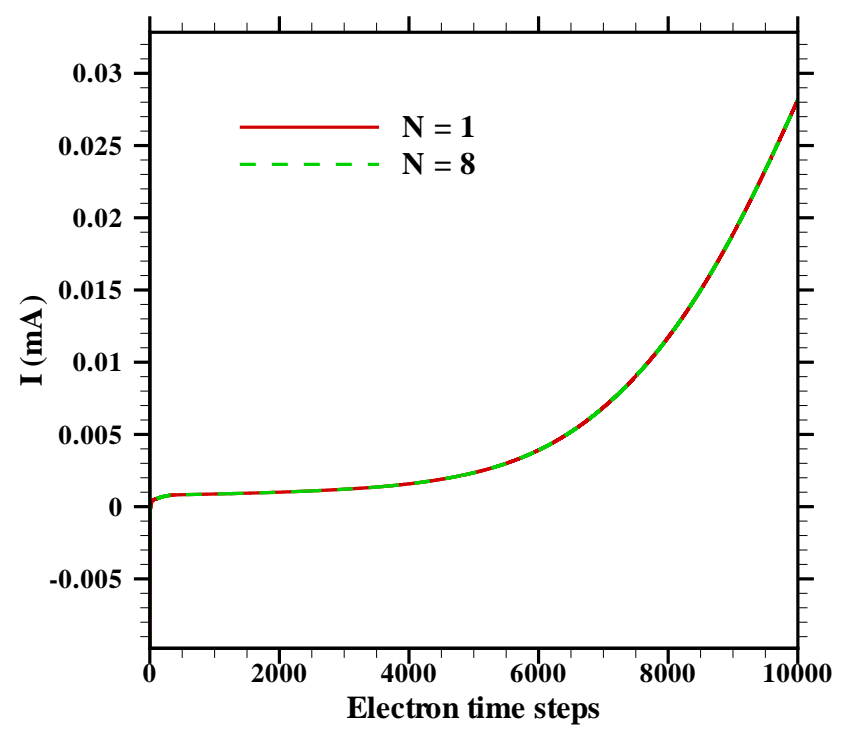

Figure 5.5: Plot of the cathode current (units: $\mathrm{mA}$ ) as a function of the number of electron time steps on $\mathrm{N}=1,8$ processors.

shown in Fig. 5.6(b). All equations are solved using the GMRES algorithm with Block Jacobi preconditioning, and Incomplete LU factorization (ILU(1)) on each block (see documentation from [37] for more details). Using ILU(1) on each block was found to result in less inner iterations compared to the $\operatorname{ILU}(0)$ case, leading to reduction in the simulation wall clock time.

Figure 5.7 shows the strong scaling curve for the air plasma validation case with a fixed mesh size of 22,920 cells $(18,000$ cells in the plasma subdomain) on 1-8 processors. Each case was run for 10,000 electron time steps, and the runs were made on a Dell HPC Linux Cluster [80] with 56 compute cores (2 quad-core processors per compute node). From Fig. 5.7 we see that specifying weights for cells in the mesh can improve the speedup of the parallel 


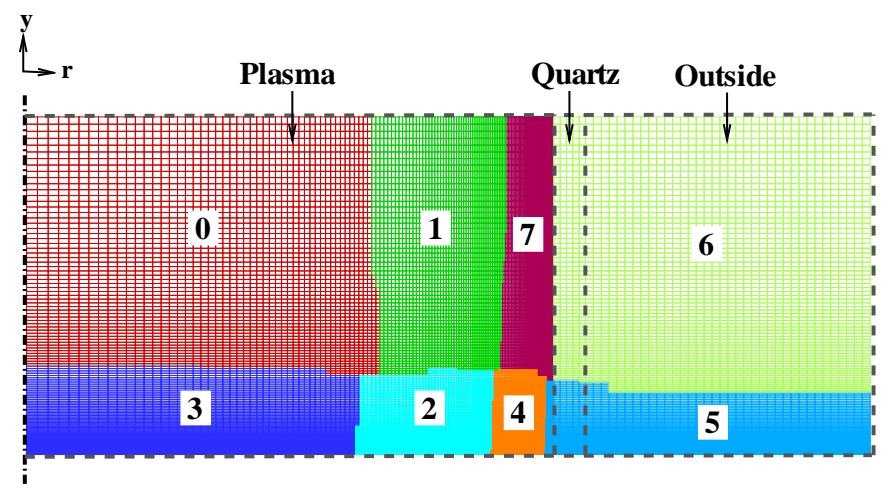

(a)

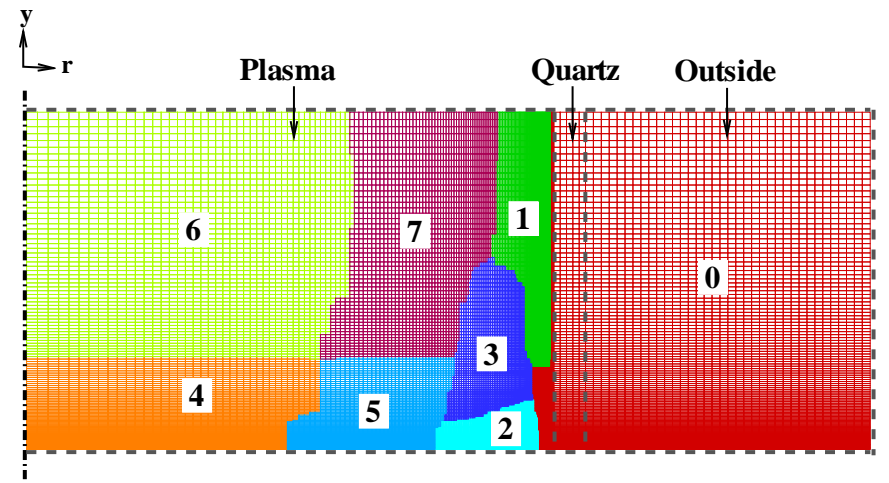

(b)

Figure 5.6: (a) Unweighted and (b) Weighted cell partitioning of the air plasma validation mesh into 8 partitions. The mesh contains 22920 cells.

implementation compared to the case where the cells in the mesh are equally weighted. For the case with 8 processors and weighted partitioning, a speedup of $\sim 6.4$ is recorded.

When multiple equations are solved on different sets of physical subdomains on a single partitioned mesh, there is bound to be a load imbalance for one (or more) of the governing equations. In the unweighted partitioning with 8 processors (Fig. 5.6(a)), partition 5 has 689 cells and partition 6 has 


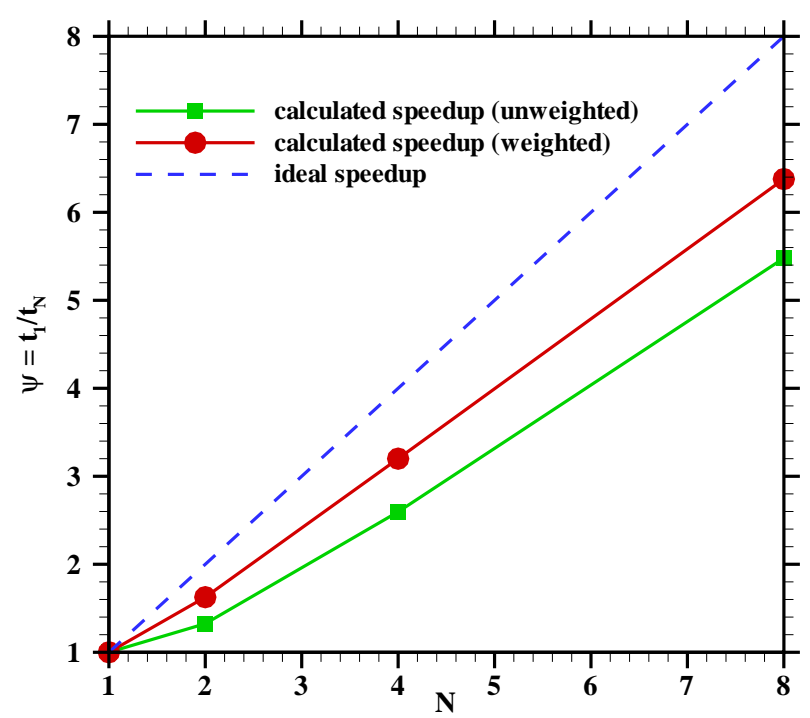

Figure 5.7: Strong scaling curves for the air plasma validation case with 22,920 cells on 1-8 processors.

121 cells on the plasma subdomain, while the other partitions have 2865 cells. Hence while the Poisson's equation is solved on a perfectly load balanced mesh, the other equations defined on the plasma subdomain alone are solved on a highly load unbalanced mesh. When the partitions are weighted (Fig. 5.6(b)), partition 0 has 1892 cells in the plasma subdomain, while the other partitions have 2301(or 2302) cells. Hence the weighting has resulted in a better balance for equations solved on the plasma subdomain alone that improves the overall performance, at the expense of poor load balance for Poisson's equation. The weighting strategy chosen in this work is based on the number of equations being solved in a given physical subdomain. However, we anticipate that weighting strategies that take into account the stiffness of the equations be- 
ing solved on a given physical subdomain and/or the amount of parallelizable computation being performed to calculate interior fluxes and source terms for each equation could lead to even better strong scaling performance.

As mentioned in Chapter 3, the serial cases require about 1-1.5 million time steps to reach a steady state that typically take about 3-4 weeks to run. With the parallel implementation with weighted partitioning, the same cases can be completed in 3-4 days, which is a significant improvement.

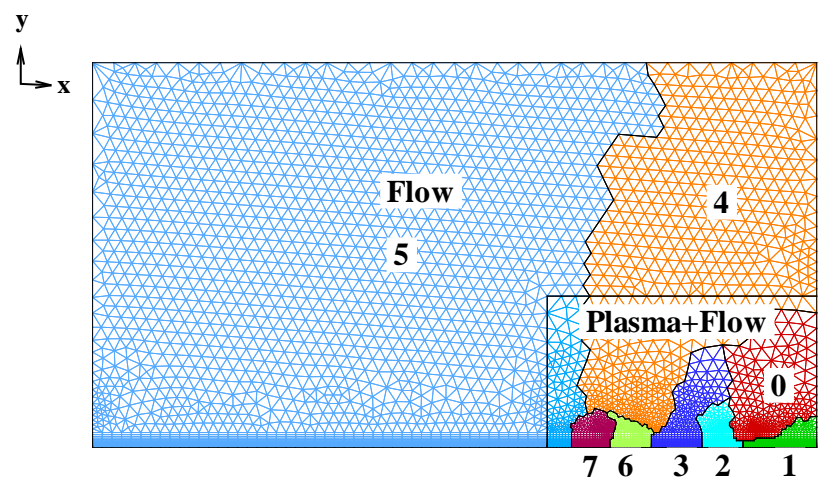

Figure 5.8: Weighted cell partitioning of the two-dimensional actuator mesh on 8 processors. The mesh contains 8504 cells with 4127 cells in the inset (plasma+flow) subdomain.

Finally we present scalability tests for the two-dimensional actuator study shown in Chapter 4. Figure 5.8 shows the same mesh used in the serial results. Following the discussion for the parallel air validation, the partition is weighted by specifying a cell weight of 15 for cells present in the inset subdomain (plasma+flow) and 1 for cells present in the outer subdomain (flow only). From an implementation viewpoint, the compressible Navier-Stokes equations can be treated as just another set of equations solved along with the 
plasma model equations, with source terms exchanged only on partitions that have both the plasma and flow models being solved (the inset subdomain).

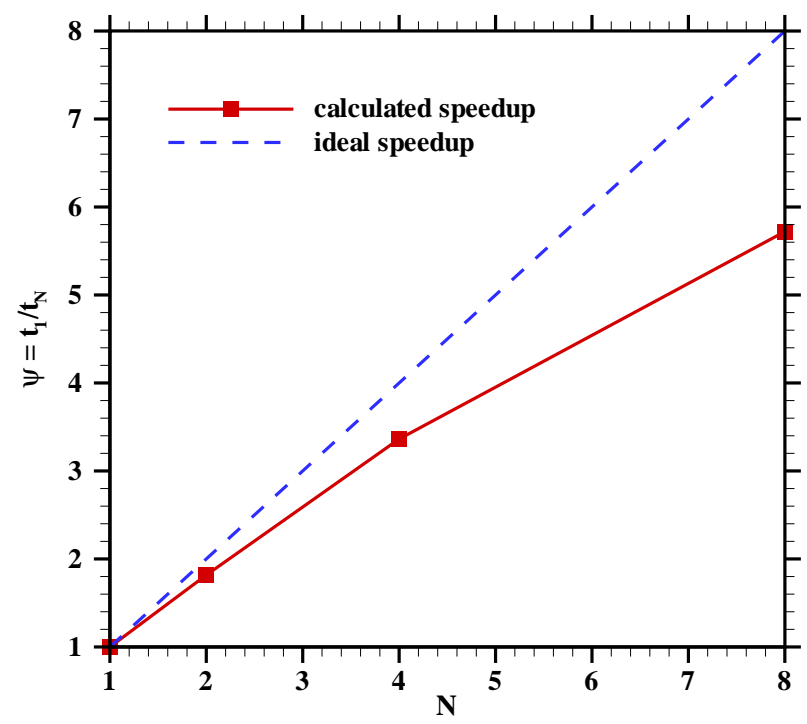

Figure 5.9: Strong scaling curve for the two-dimensional actuator case with 8504 cells on $1-8$ processors.

From Fig. 5.9 we see that with cell weighted partitioning, a speedup of $\sim 5.7$ is recorded for $\mathrm{N}=8$ processors. The speedup calculations were performed by running each case $\mathrm{N}=1,2,4$ and 8 for 1000 electron time steps. One of the reasons that the algorithm scales reasonably well for a relatively small mesh $(8504$ cells) is that the number of dependent variables that are present in each cell is large due to the number of species in the plasma chemistry model. The amount of "serial" work being done in calculating face fluxes and matrix coefficient contributions is significant despite the relatively small mesh size leading to better parallel performance. 


\section{Chapter 6}

\section{Three-Dimensional Pin Actuator Study}

In this chapter we present simulation results of the three-dimensional surface plasma discharge performed with the parallel implementation discussed in Chapter 5 for the case where no flow is present. The schematic of the pin actuator geometry is shown in Fig. 6.1. The electrode diameter is $2.4 \mathrm{~mm}$ and the inter-electrode gap is $7.62 \mathrm{~mm}$, which match the dimensions used in previous experiments [14].

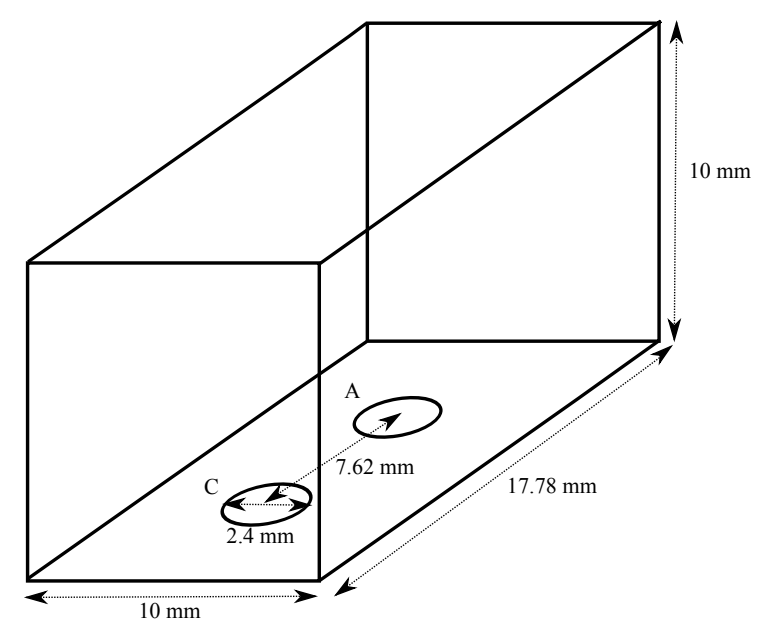

Figure 6.1: The circular pin-electrode actuator schematic is shown. The source voltage is applied at the cathode $(\mathrm{C})$, with a resistance in series. The anode (A) is grounded.

The mesh used in the simulations is shown in Fig. 6.2 and contains 
343,010 cells. While hexahedral and prismatic cells are used near the surface in order to resolve the sheath, a tetrahedral mesh is used in the farfield. In the transition region between the hexahedral and tetrahedral cells, pyramids are present to stitch the hexahedral cells to the tetrahedral cells. On the surface, an annular mesh is generated in the region around each electrode to provide better control over the mesh spacing. As noted previously in Chapter 4, the interface of the electrodes and the dielectric is where the highest electric fields are encountered and hence the mesh needs to be better resolved in those regions.

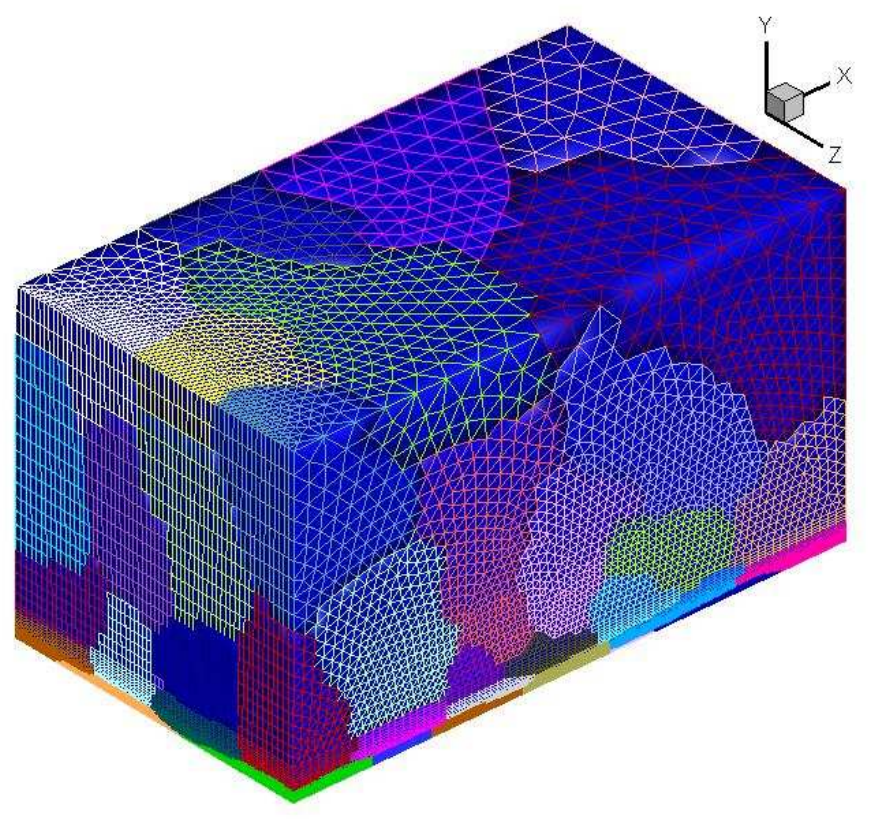

Figure 6.2: The computational mesh used for the three-dimensional actuator simulations, divided into 128 partitions. The mesh contains a total of 343,010 cells.

The conditions for the simulations are the same as those used for the 
two-dimensional surface discharge case in the absence of flow: a cathode source voltage of $-1000 \mathrm{~V}$, and a series resistance of $1 \mathrm{k} \Omega$. The ambient pressure is fixed at 18 Torr, and the ambient temperature is $300 \mathrm{~K}$. The simulations are initially run in parallel on Ranger [79] for 100 electron time steps on $\mathrm{N}=1-128$ processors to find the strong scaling curve.

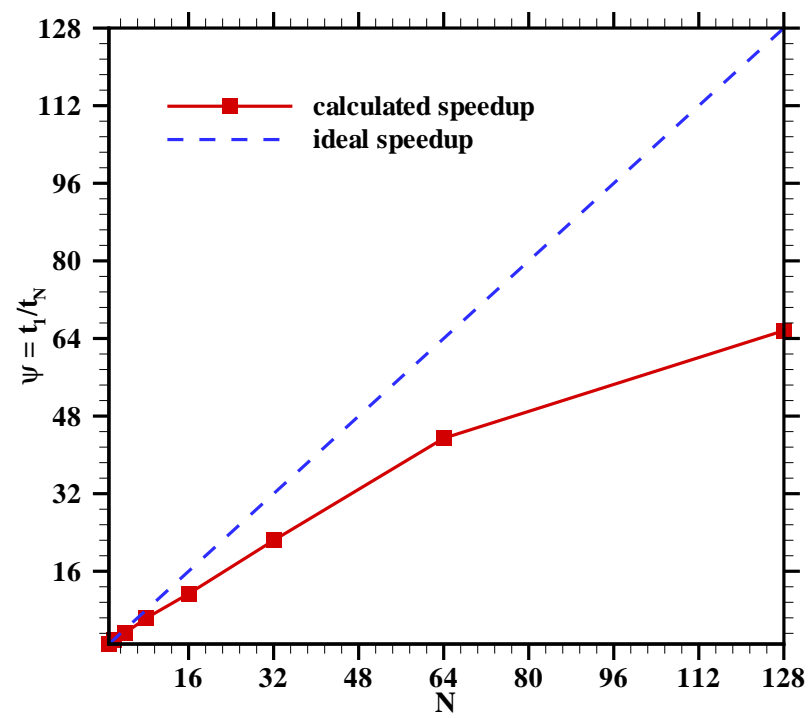

Figure 6.3: Strong scaling curve for the three-dimensional actuator simulations with 343,010 cells on 1-128 processors.

In Fig. 6.3, the time used to calculate the speedup $\Psi$ is the wall clock time taken to solve 100 electron time steps and does not include the time taken to read and partition the mesh and other input files. From the figure we see that the algorithm scales linearly till $\mathrm{N}=64$ processors, and the slope reduces between $\mathrm{N}=64$ and $\mathrm{N}=128$ processors. The reduction in slope is due to the increased communication cost and reduced computation being performed per 
processor (the total problem size is fixed). Nevertheless, the $\mathrm{N}=128$ processor case is about 1.5 times faster than the $\mathrm{N}=64$ processor case, and is used for the simulations. In terms of absolute time, the $\mathrm{N}=1$ processor case takes 193 minutes to run for 100 electron time steps, while the $\mathrm{N}=128$ processor case takes about 3 minutes ( $\sim 65$ times faster). To reach a steady state, typically $10^{5}-10^{6}$ electron time steps are required.

The three-dimensional structure of the discharge is observed in Fig. 6.4. Contours of the electrostatic potential on the electrodes and the dielectric surface are also shown. From the isosurface of $\mathrm{O}_{2}^{+}$at $3 \times 10^{18} \mathrm{~m}^{-3}$, we see that the $\mathrm{O}_{2}^{+}$density covers the cathode above the cathode sheath, and attached to the leading edge of the anode. The discharge current in this case is about 25 $\mathrm{mA}$, which is similar to the low-power diffuse discharge observed in previous experiments [14]. In the two-dimensional studies, the current recorded in the absence of flow was $\sim 10 \mathrm{~mA}$ based on $1 \mathrm{~mm}$ depth. Given that the electrode diameter is $2.4 \mathrm{~mm}$, the current observed in the three-dimensional case is similar to that observed in the two-dimensional case.

Next, we examine the charge density contours of the various dominant positively and negatively charged ions in the discharge on the $\mathrm{z}=0$ plane and compare the results to the two-dimensional results of Section 4.1. In Fig. 6.5, the charge densities of electrons and $\mathrm{O}^{-}$ions are shown. Comparing Figs. 6.5(a) and 4.3(a), we see that the electron density near the anode is higher in the three-dimensional case. Given similar currents and power, in the three-dimensional case, the electron current at the anode is concentrated over a smaller area corresponding to the region where the plasma is attached to the 


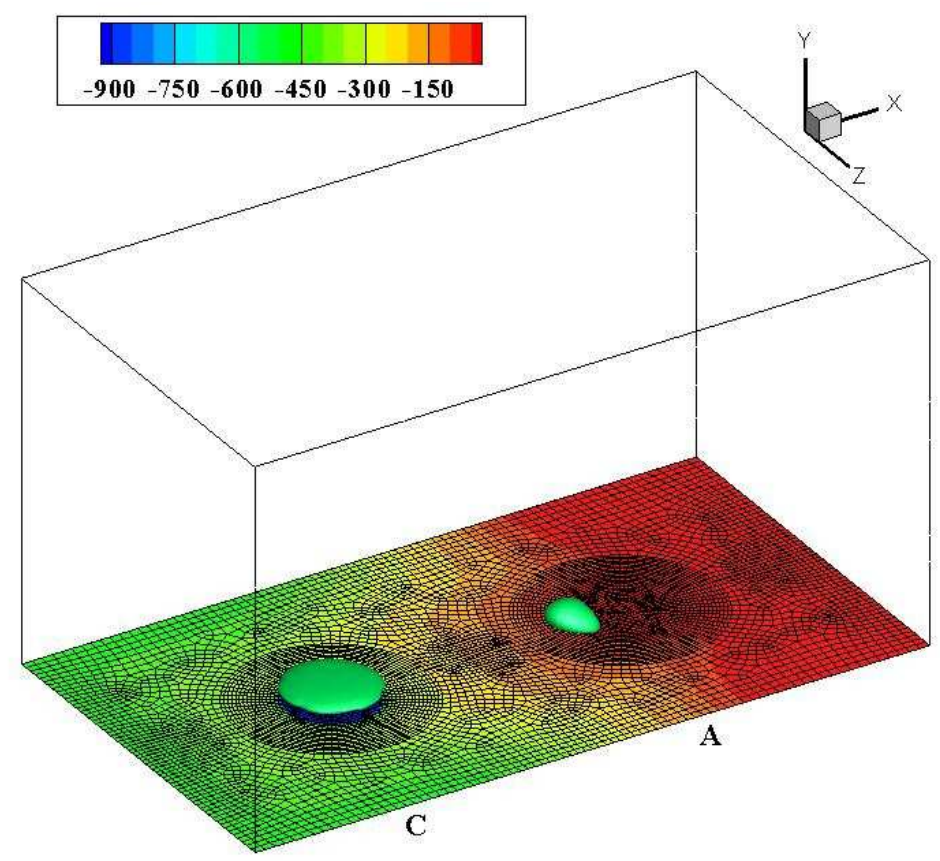

Figure 6.4: Three-dimensional structure of the discharge in the pin-electrode configuration. The isosurface of $\mathrm{O}_{2}^{+}$at a value of $3 \times 10^{18} \mathrm{~m}^{-3}$ is shown. Contours of the potential (units: V) on the electrodes and the dielectric surface are also shown.

anode, resulting in a higher electron density near the anode. Similar results are observed for $\mathrm{O}^{-}$ions from Fig. 6.5(b), and for $\mathrm{O}_{2}^{+}$ions from Fig. 6.6(b). As was observed in the two-dimensional case, the plasma is electronegative with the $\mathrm{O}^{-}$ion density about three times higher than the electron density. The $\mathrm{N}_{2}^{+}$ion is seen to be the dominant positive ion species in the cathode sheath from Fig. 6.6(a), with a second peak near the anode. In the case of $\mathrm{N}_{4}^{+}$ions, the peak ion density is observed in the vicinity of the anode, and a peak of $\mathrm{O}_{4}^{+}$ion density is found downstream of the cathode edge. Overall, we note that there are several qualitative and quantitative similarities between the three-dimensional and two-dimensional results in this case. 
Finally, we examine the gas temperature contours for the pin electrode discharge. We observe that the peak gas temperature in Fig. 6.8 is $~ 1000 \mathrm{~K}$, which is lower than the temperature of $\sim 1180 \mathrm{~K}$ observed in Fig. 4.6. The same assumptions made in the two-dimensional case are used here, i.e. ion Joule heating thermalization factor $\eta_{\mathrm{Th}}=0.25$, along with the assumption that all the energy deposited into the excited states of the neutral gas is eventually transferred into the gas energy (translational) pool. We have already noted that similar discharge powers are observed in the two-dimensional and three-dimensional cases. The lower gas temperature observed in the threedimensional discharge could be attributed to a three-dimensional relief effect. In the plane actuator case (Fig. 4.6), the power deposited into the gas energy pool is conducted in the streamwise $(\mathrm{x})$ and wall-normal $(\mathrm{y})$ directions, with no conduction in the spanwise $(\mathrm{z})$ direction. In the pin actuator case, the power deposited into the gas energy pool in all three coordinate directions, leading to a lower peak gas temperature observed in Fig. 6.8. 


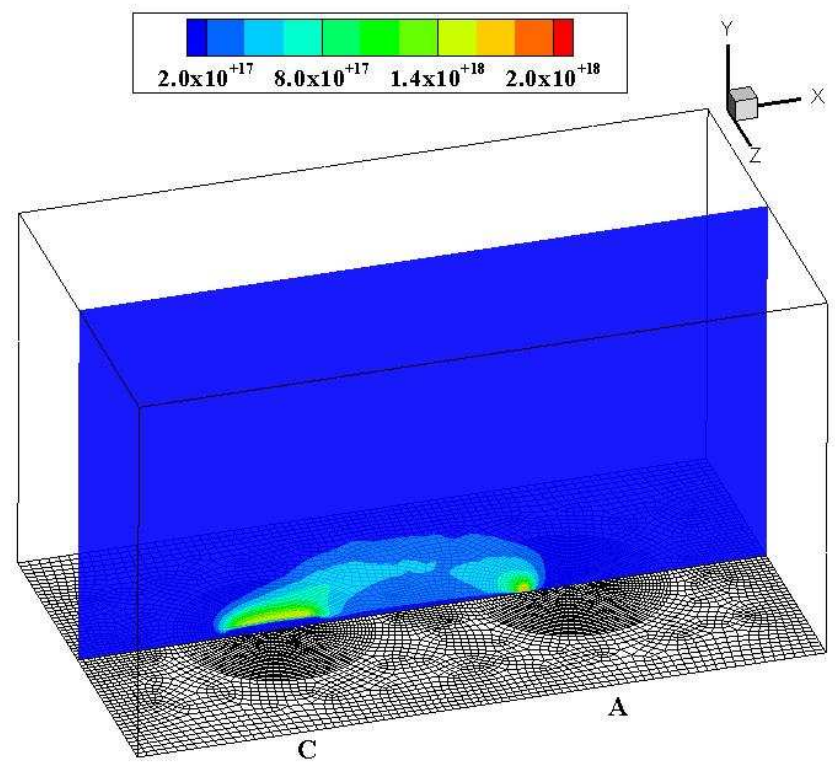

(a)

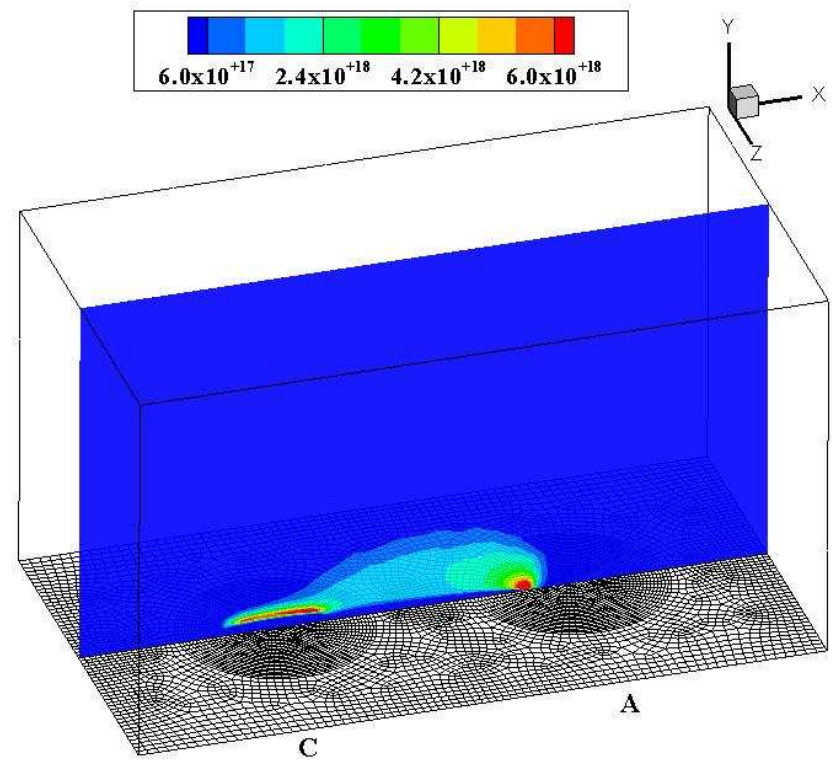

(b)

Figure 6.5: Species number densities (units: $\mathrm{m}^{-3}$ ) of $(\mathrm{a}) \mathrm{e}^{-}$, and (b) $\mathrm{O}^{-}$ions in the surface plasma discharge (no bulk flow) in the pin-electrode configuration. The cathode source voltage is $-1000 \mathrm{~V}$, and the ambient pressure is fixed at 18 Torr. 


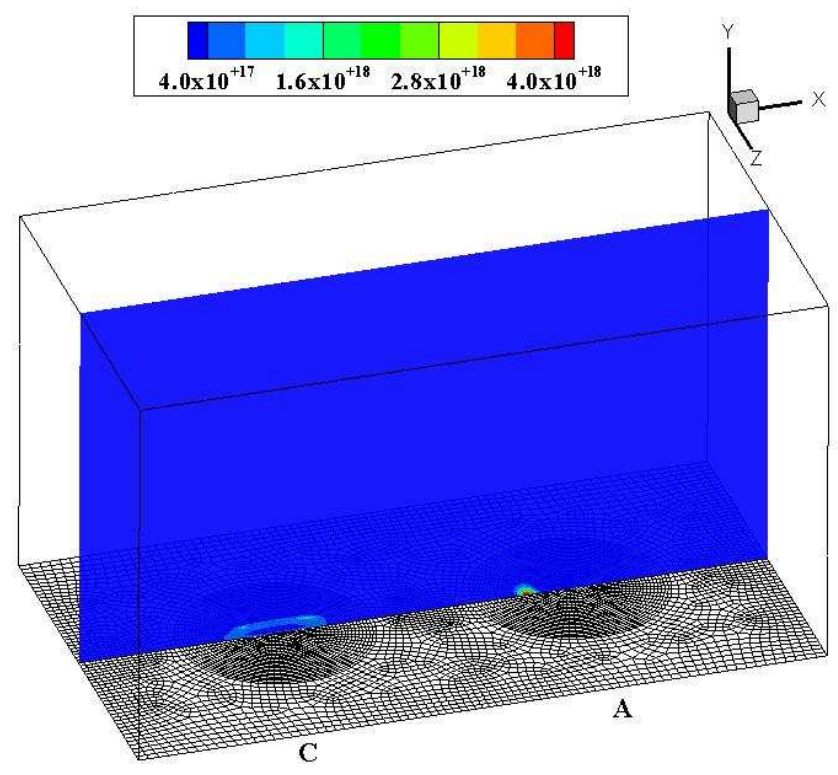

(a)

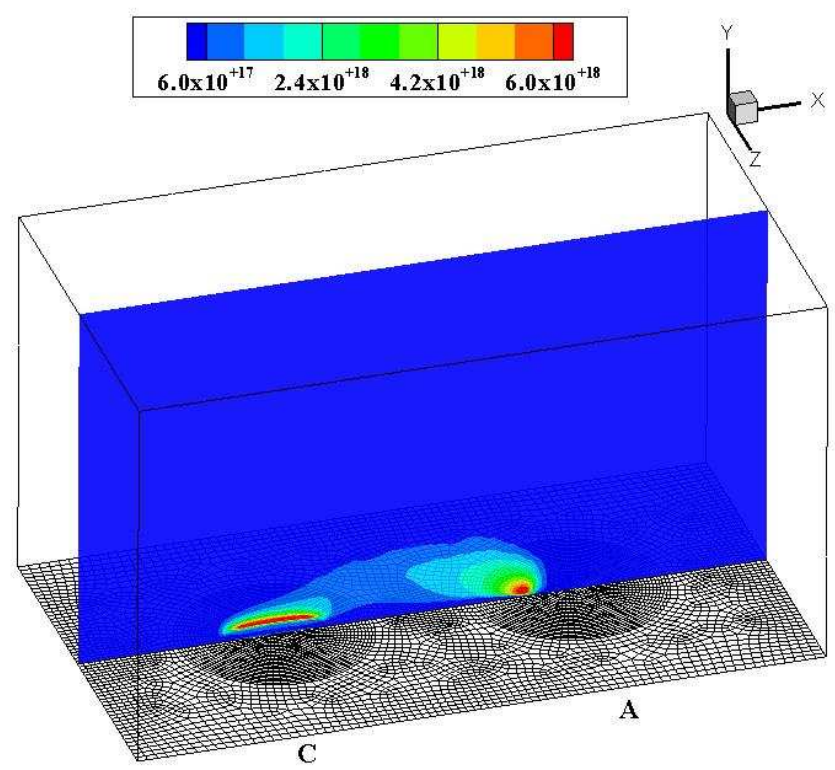

(b)

Figure 6.6: Species number densities (units: $\mathrm{m}^{-3}$ ) of $(\mathrm{a}) \mathrm{N}_{2}^{+}$, and (b) $\mathrm{O}_{2}^{+}$ions in the surface plasma discharge (no bulk flow) in the pin-electrode configuration. The cathode source voltage is $-1000 \mathrm{~V}$, and the ambient pressure is fixed at 18 Torr. 


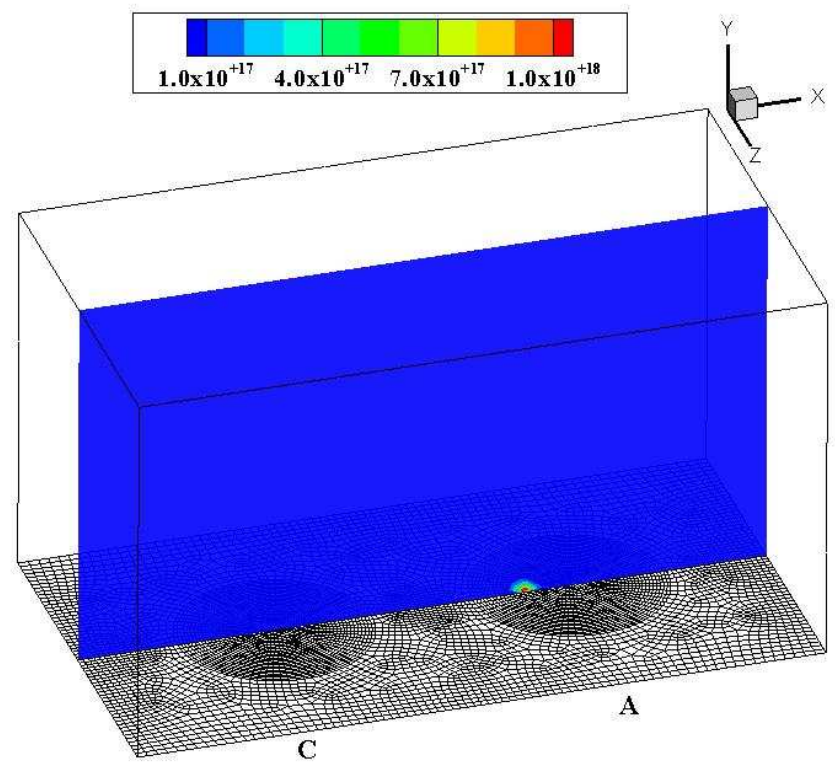

(a)

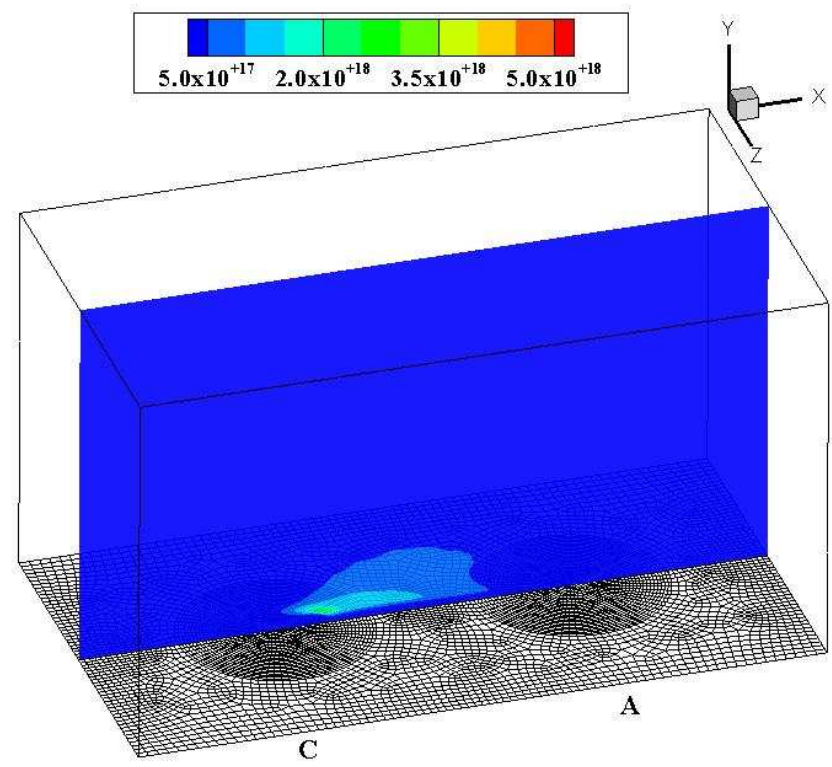

(b)

Figure 6.7: Species number densities (units: $\mathrm{m}^{-3}$ ) of $(\mathrm{a}) \mathrm{N}_{4}^{+}$, and (b) $\mathrm{O}_{4}^{+}$ions in the surface plasma discharge (no bulk flow) in the pin-electrode configuration. The cathode source voltage is $-1000 \mathrm{~V}$, and the ambient pressure is fixed at 18 Torr. 


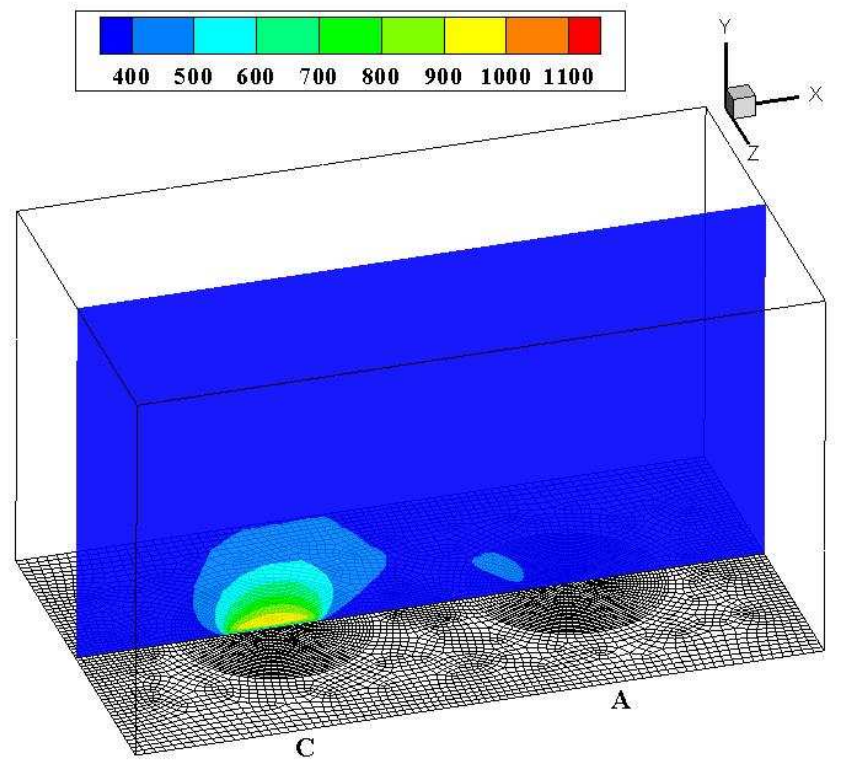

Figure 6.8: Gas temperature (units: K) in the surface plasma discharge (no bulk flow) on the center-line in the pin-electrode configuration. The cathode source voltage is $-1000 \mathrm{~V}$, and the ambient pressure is fixed at 18 Torr. 


\section{Chapter 7}

\section{Conclusion}

Computational simulations of surface plasma discharges in the presence of supersonic flow are presented in this work. The plasma model is based on a self-consistent, multi-species, multi-temperature, finite-rate chemistry, continuum description of the plasma. A reduced air plasma model suitable for multi-dimensional applications with 11 species and 21 gas-phase chemical reactions is presented and validated in Chapter 3 using experimental results from the literature.

The air plasma chemistry model is found to provide reasonably good prediction of experimental current-voltage characteristics of the discharge, and spatial profiles of the electron temperature and positive ion number densities. For pressures of order 1 Torr, $\mathrm{O}_{2}^{+}$and $\mathrm{N}_{2}^{+}$are the dominant positive ion species in the discharge, and the concentration of $\mathrm{O}^{-}$is comparable to electron concentration. The air plasma can therefore be characterized as moderately electronegative. The two-dimensional structure of the discharge obtained from the computations is found to be in agreement with qualitative observations from the experiments.

The plasma model is self-consistently coupled with a compressible NavierStokes solver to study the effect of the plasma on the flow and the counter-effect 
of the flow on the plasma. The air plasma chemistry model is then used to study the effect of the surface plasma discharge on $M=3$ supersonic flow at freestream pressure 18 Torr in two dimensions. The species concentrations and the gas temperature are examined in the absence and presence of bulk supersonic flow. From the results presented in Chapter 4 , the $\mathrm{O}^{-}$concentration is found to exceed electron concentration in the pressure range 1-20 Torr, and $\mathrm{O}_{2}^{-}$concentrations are at least two orders of magnitude smaller over the pressure range considered.

From the computations in Chapter 4, the peak gas temperature is found to be $1180 \mathrm{~K}$ with the surface plasma alone, and $830 \mathrm{~K}$ in the presence of supersonic flow. The dominant positive ion species in the cathode sheath is $\mathrm{N}_{2}^{+}$, while $\mathrm{O}_{2}^{+}$is the dominant positive ion species in the bulk plasma when no supersonic flow is present. In the bulk plasma, an ion-ion core characteristic of electronegative plasmas is found. With supersonic flow, $\mathrm{O}_{2}^{+}$and $\mathrm{O}_{4}^{+}$are found to be the dominant positive ion species in the bulk plasma while $\mathrm{N}_{2}^{+}$ remains dominant in the cathode sheath. Hence different ion species are found to be dominant in the absence and presence of supersonic flow, highlighting the importance of including finite-rate chemistry effects in discharge models for understanding plasma actuator physical phenomena.

The effects of varying the discharge voltage and the freestream pressure are studied in Chapter 4 . The discharge model is found to be able to capture the transition for normal to abnormal mode glow discharges. Electrode polarity effects are investigated, and the cathode upstream actuation is found to be stronger than the actuation strength with the cathode downstream, which 
is consistent with experimental findings of several groups. The cathode downstream discharge is found to be more electronegative compared to the cathode upstream case. The qualitative structure of the discharge is found to be different when the cathode is located downstream compared to when the cathode is located upstream. In the cathode upstream case, the gas temperature in the bulk plasma region is comparatively higher owing to convection of the heat deposited in the cathode sheath by ion Joule heating. The gas temperature field strongly influences the formation of cluster ions such as $\mathrm{O}_{4}^{+}$, and explains differences in the discharge structure when the electrode polarity is reversed.

The fact that the plasma is electronegative under the present conditions and the electronegativity has a strong influence on the discharge potential and electron temperature profiles indicates that a pure nitrogen plasma model is not a good surrogate for an air plasma model as used in several recent modeling studies.

The time-splitting approach used to alleviate temporal stiffness in the model described in Section 2.1.3 is an important factor in obtaining the steady state simulation results presented in Chapters 3 and 4 . Despite the improved efficiency of the time-splitting approach, about 3-4 weeks of run time on a high-performance blade server (serial computation) is required for the current air chemistry model (11 species) and computational mesh sizes ( 10,000 cells). Hence there are practical limitations on the grid size and number of species that can be included in the air chemistry model. Large simulation times also make the study of sensitivity to model parameters more difficult, which motivates the need for further algorithmic improvements for future studies in addition 
to the parallelization of continuum plasma models. Moreover, in experiments with plasma actuators, circular pin shaped electrodes are used which result in discharges that are inherently three-dimensional. A parallel implementation of the plasma and flow models was therefore developed as part of this research and is discussed in Chapter 5 .

The parallel implementation allows the solution of the flow and plasma models on multiple physical subdomains. The verification and speedup characteristics of the parallel model is discussed in Chapter 5. While solving problems with multiple equations on multiple physical subdomains, some equations end up being solved on a poorly balanced mesh. Supplying weights for mesh cells based on the number of equations being solved in a given physical subdomain during mesh partitioning is found to be an effective strategy to improve the parallel performance of the model.

In Chapter 6, the parallel model is used to study the three-dimensional plasma actuator configuration with circular pin electrodes in the absence of bulk supersonic flow. The three-dimensional structure of the discharge is captured by the model, and in general qualitative agreement is found between the three-dimensional and corresponding two-dimensional simulations for the same operating parameters. 
Appendices 


\section{Appendix A}

\section{Electron impact excitation, ionization and attachment rate coefficients}

The zero-dimensional electron species Boltzmann equation solver BOLSIG $+[45]$ is used off-line to determine electron impact reaction rate coefficients and the mean electron energy $\left(=\frac{3}{2} T_{e}\right)$ as a function of the reduced electric field $\left(\mathrm{E} / \mathrm{N}_{g}\right)$ over a range of values for $\mathrm{E} / \mathrm{N}_{g}$. Cross sections for electron impact processes, the background gas composition and background gas temperature serve as inputs to the model. In the present work, a $79 \% \mathrm{~N}_{2}$ and $21 \% \mathrm{O}_{2}$ is used as representative of air. The cross section data for electron impact reactions with $\mathrm{N}_{2}$ and $\mathrm{O}_{2}$ obtained from the literature is listed in Appendix B. For the given range of $\left(\mathrm{E} / \mathrm{N}_{g}\right)$, BOLSIG + calculates the EEDFs, the reaction rate coefficients and electron transport properties as a function of the mean electron

energy $\left(=\frac{3}{2} T_{e}\right)$ are obtained as outputs. The rate coefficients are subsequently mapped with the electron temperature $T_{e}$ to create tabulated values of the rate coefficients as function of the electron temperatures. The rate coefficients (in units of $\mathrm{m}^{3} \mathrm{~s}^{-1}$ ) for reactions $\mathrm{G}_{1}, \mathrm{G}_{2}, \mathrm{G}_{16}, \mathrm{G}_{17}, \mathrm{G}_{18}, \mathrm{G}_{20}$ and $\mathrm{G}_{21}$ obtained using this procedure are shown in Fig. 3.1. The lines in Fig. 3.1 represent curve fits to the data obtained from BOLSIG+, and are listed below (for $T_{e}$ in Kelvin unit): 


$$
\begin{array}{r}
\mathrm{G}_{1}: \text { if }\left(T_{e}>1160\right) \exp \left(-\left(2.418 \times 10^{26} / T_{e}^{6}\right)+\left(7.360 \times 10^{22} / T_{e}^{5}\right)\right. \\
-\left(8.507 \times 10^{18} / T_{e}^{4}\right)+\left(4.409 \times 10^{14} / T_{e}^{3}\right)-\left(7.475 \times 10^{9} / T_{e}^{2}\right) \\
\left.-\left(2.927 \times 10^{5} / T_{e}\right)-29.543\right)
\end{array}
$$

$$
\begin{array}{r}
\mathrm{G}_{2}: \operatorname{if}\left(T_{e}>1160\right) \exp \left(-\left(1.542 \times 10^{35} / T_{e}^{8}\right)+\left(6.401 \times 10^{31} / T_{e}^{7}\right)\right. \\
-\left(1.074 \times 10^{28} / T_{e}^{6}\right)+\left(9.350 \times 10^{23} / T_{e}^{5}\right)-\left(4.507 \times 10^{19} / T_{e}^{4}\right)
\end{array}
$$$$
\left.+\left(1.166 \times 10^{15} / T_{e}^{3}\right)-\left(1.127 \times 10^{10} / T_{e}^{2}\right)-\left(2.924 \times 10^{5} / T_{e}\right)-29.380\right)
$$

$$
\begin{array}{r}
\mathrm{G}_{16}: \operatorname{if}\left(T_{e}>5802\right) \exp \left(-\left(1.033 \times 10^{35} / T_{e}^{8}\right)+\left(4.520 \times 10^{31} / T_{e}^{7}\right)\right. \\
-\left(8.184 \times 10^{27} / T_{e}^{6}\right)+\left(7.963 \times 10^{23} / T_{e}^{5}\right)-\left(4.547 \times 10^{19} / T_{e}^{4}\right) \\
\left.+\left(1.564 \times 10^{15} / T_{e}^{3}\right)-\left(3.148 \times 10^{10} / T_{e}^{2}\right)+\left(2.618 \times 10^{5} / T_{e}\right)-34.711\right)
\end{array}
$$

$$
\begin{array}{r}
\mathrm{G}_{17}: \operatorname{if}\left(T_{e}>5802\right) \exp \left(-\left(1.113 \times 10^{39} / T_{e}^{9}\right)+\left(5.213 \times 10^{35} / T_{e}^{8}\right)\right. \\
-\left(1.032 \times 10^{32} /\left(T_{e}^{7}\right)\right)+\left(1.124 \times 10^{28} / T_{e}^{6}\right)-\left(7.368 \times 10^{23} / T_{e}^{5}\right) \\
+\left(2.962 \times 10^{19} / T_{e}^{4}\right)-\left(7.175 \times 10^{14} / T_{e}^{3}\right)+\left(1.068 \times 10^{10} / T_{e}^{2}\right) \\
\left.-\left(1.956 \times 10^{5} / T_{e}\right)-30.382\right)
\end{array}
$$

$$
\begin{array}{r}
\mathrm{G}_{18}: \operatorname{if}\left(T_{e}>5802\right) \exp \left(-\left(8.287 \times 10^{34} / T_{e}^{8}\right)+\left(3.775 \times 10^{31} / T_{e}^{7}\right)\right. \\
-\left(7.258 \times 10^{27} / T_{e}^{6}\right)+\left(7.669 \times 10^{23} / T_{e}^{5}\right)-\left(4.862 \times 10^{19} / T_{e}^{4}\right) \\
\left.+\left(1.893 \times 10^{15} / T_{e}^{3}\right)-\left(4.466 \times 10^{10} / T_{e}^{2}\right)+\left(5.695 \times 10^{5} / T_{e}\right)-40.809\right)
\end{array}
$$




$$
\begin{array}{r}
\mathrm{G}_{20}: \text { if }\left(T_{e}>5802\right) \exp \left(-\left(6.059 \times 10^{33} / T_{e}^{8}\right)+\left(3.727 \times 10^{30} / T_{e}^{7}\right)\right. \\
-\left(9.137 \times 10^{26} / T_{e}^{6}\right)+\left(1.146 \times 10^{23} / T_{e}^{5}\right)-\left(7.818 \times 10^{18} / T_{e}^{4}\right) \\
\left.+\left(2.722 \times 10^{14} / T_{e}^{3}\right)-\left(2.379 \times 10^{9} / T_{e}^{2}\right)-\left(1.601 \times 10^{5} / T_{e}\right)-27.366\right) \\
\mathrm{G}_{21}: \operatorname{if}\left(T_{e}>5802\right) \exp \left(-\left(1.683 \times 10^{25} / T_{e}^{6}\right)+\left(8.847 \times 10^{21} / T_{e}^{5}\right)\right. \\
-\left(1.796 \times 10^{18} / T_{e}^{4}\right)+\left(1.800 \times 10^{14} / T_{e}^{3}\right)-\left(9.351 \times 10^{9} / T_{e}^{2}\right) \\
\left.-\left(1.997 \times 10^{5} / T_{e}\right)-34.314\right)
\end{array}
$$

As mentioned in Section 3.1, the reaction rate coefficient for $\mathrm{G}_{20}$ is calculated by "lumping" reactions of the form: $\mathrm{e}^{-}+\mathrm{N}_{2} \rightarrow \mathrm{e}^{-}+\mathrm{N}_{2}^{*}$ where $\mathrm{N}_{2}^{*}$ includes the species $\mathrm{N}_{2}($ rot $), \mathrm{N}_{2}(\mathrm{X}, \mathrm{v}=1-8), \mathrm{N}_{2}\left(\mathrm{~A}^{3} \Sigma_{u}^{+}\right), \mathrm{N}_{2}\left(\mathrm{~B}^{3} \Pi_{g}\right), \mathrm{N}_{2}\left(\mathrm{~W}^{3} \Delta_{u}\right)$, $\mathrm{N}_{2}\left(\mathrm{~B}^{\prime 3} \Sigma_{u}^{-}\right), \mathrm{N}_{2}\left(\mathrm{a}^{\prime 1} \Sigma_{u}^{-}\right), \mathrm{N}_{2}\left(\mathrm{a}^{1} \Pi_{g}\right), \mathrm{N}_{2}\left(\mathrm{w}^{1} \Delta_{u}\right), \mathrm{N}_{2}\left(\mathrm{C}^{3} \Pi_{u}\right), \mathrm{N}_{2}\left(\mathrm{E}^{3} \Sigma_{g}^{+}\right), \mathrm{N}_{2}\left(\mathrm{a}^{\prime \prime 1} \Sigma_{g}^{+}\right)$, and sum of singlet states [61]. The contribution of the above reactions to the inelastic collision loss term in 2.5 can be written as

$$
-e \sum_{i \in N_{2}^{\mathrm{EXCI}}} \Delta E_{i}^{e} k_{i} n_{e} n_{N_{2}} \equiv-e \Delta E_{G_{20}}^{e} k_{G_{20}} n_{e} n_{N_{2}}
$$

By setting $\Delta E_{G_{20}}^{e}=1 \mathrm{eV}$, we can write the "lumped" reaction rate coefficient $k_{G_{20}}=\sum_{i \in N_{2}^{\mathrm{EXCI}}} \Delta E_{i}^{e} k_{i}$. Similarly, we can calculate $k_{G_{21}}$. 


\section{Appendix B}

\section{Cross section data for electron impact collisions with $\mathrm{N}_{2}$ and $\mathrm{O}_{2}$}

The integral cross section data for electron collisions with $\mathrm{N}_{2}$ and $\mathrm{O}_{2}$ used as input to BOLSIG+ for the EEDF, rate coefficient and transport property calculations is listed here. For $\mathrm{N}_{2}$, the cross section data for elastic collisions, $\mathrm{N}_{2}$ excited states $\mathrm{N}_{2}(\mathrm{rot}), \mathrm{N}_{2}(\mathrm{X}, \mathrm{v}=1-8), \mathrm{N}_{2}\left(\mathrm{~A}^{3} \Sigma_{u}^{+}\right), \mathrm{N}_{2}\left(\mathrm{~B}^{3} \Pi_{g}\right)$, $\mathrm{N}_{2}\left(\mathrm{~W}^{3} \Delta_{u}\right), \mathrm{N}_{2}\left(\mathrm{~B}^{\prime 3} \Sigma_{u}^{-}\right), \mathrm{N}_{2}\left(\mathrm{a}^{\prime 1} \Sigma_{u}^{-}\right), \mathrm{N}_{2}\left(\mathrm{a}^{1} \Pi_{g}\right), \mathrm{N}_{2}\left(\mathrm{w}^{1} \Delta_{u}\right), \mathrm{N}_{2}\left(\mathrm{C}^{3} \Pi_{u}\right), \mathrm{N}_{2}\left(\mathrm{E}^{3} \Sigma_{g}^{+}\right)$, $\mathrm{N}_{2}\left(\mathrm{a}^{\prime \prime 1} \Sigma_{g}^{+}\right)$, sum of singlet states [61], and ionization is obtained from [62, 61]. For $\mathrm{O}_{2}$, the cross section data for elastic collisions, excited states $\mathrm{O}_{2}(\mathrm{rot})$, $\mathrm{O}_{2}($ vsum $), \mathrm{O}_{2}\left(\mathrm{a}^{1} \Delta_{g}\right), \mathrm{O}_{2}\left(\mathrm{~b}^{1} \Sigma_{u}^{+}\right), \mathrm{O}_{2}(4.5)$, dissociation, dissociative attachment and ionization is obtained from [64, 63].

Table B.1: Cross section data for $\mathrm{N}_{2}$ Elastic $\left(\Delta E^{e}=1.95 e-005 \mathrm{eV}\right)$

\begin{tabular}{|c|c|}
\hline Energy (eV) & Cross Section $\left(\mathbf{m}^{2}\right)$ \\
\hline 0.0 & $1.1 \mathrm{e}-020$ \\
0.001 & $1.36 \mathrm{e}-020$ \\
0.002 & $1.49 \mathrm{e}-020$ \\
0.003 & $1.62 \mathrm{e}-020$ \\
0.005 & $1.81 \mathrm{e}-020$ \\
0.007 & $2 \mathrm{e}-020$ \\
0.0085 & $2.1 \mathrm{e}-020$ \\
0.01 & $2.19 \mathrm{e}-020$ \\
0.015 & $2.55 \mathrm{e}-020$ \\
0.02 & $2.85 \mathrm{e}-020$ \\
0.03 & $3.4 \mathrm{e}-020$ \\
0.04 & $3.85 \mathrm{e}-020$ \\
0.05 & $4.33 \mathrm{e}-020$ \\
0.07 & $5.1 \mathrm{e}-020$ \\
0.1 & $5.95 \mathrm{e}-020$ \\
\hline \multicolumn{2}{|c}{ Continued on next page } \\
\hline
\end{tabular}


Table B.1 - continued from previous page

\begin{tabular}{|c|c|}
\hline Energy (eV) & Cross Section $\left(\mathrm{m}^{2}\right)$ \\
\hline 0.12 & $6.45 \mathrm{e}-020$ \\
\hline 0.15 & $7.1 \mathrm{e}-020$ \\
\hline 0.17 & $7.4 \mathrm{e}-020$ \\
\hline 0.2 & $7.9 \mathrm{e}-020$ \\
\hline 0.25 & $8.5 \mathrm{e}-020$ \\
\hline 0.3 & $9 \mathrm{e}-020$ \\
\hline 0.35 & $9.4 \mathrm{e}-020$ \\
\hline 0.4 & $9.7 \mathrm{e}-020$ \\
\hline 0.5 & $9.9 \mathrm{e}-020$ \\
\hline 0.7 & $1 e-019$ \\
\hline 1.0 & $1 e-019$ \\
\hline 1.2 & $1.04 \mathrm{e}-019$ \\
\hline 1.3 & $1.1 \mathrm{e}-019$ \\
\hline 1.5 & $1.2 \mathrm{e}-019$ \\
\hline 1.7 & $1.38 \mathrm{e}-019$ \\
\hline 1.9 & $1.96 \mathrm{e}-019$ \\
\hline 2.1 & $2.7 \mathrm{e}-019$ \\
\hline 2.2 & $2.85 \mathrm{e}-019$ \\
\hline 2.5 & 3e-019 \\
\hline 2.8 & $2.8 \mathrm{e}-019$ \\
\hline 3.0 & $2.17 \mathrm{e}-019$ \\
\hline 3.3 & $1.72 \mathrm{e}-019$ \\
\hline 3.6 & $1.47 \mathrm{e}-019$ \\
\hline 4.0 & $1.26 \mathrm{e}-019$ \\
\hline 4.5 & $1.13 \mathrm{e}-019$ \\
\hline 5.0 & $1.09 \mathrm{e}-019$ \\
\hline 6.0 & $1.04 \mathrm{e}-019$ \\
\hline 7.0 & $1.01 \mathrm{e}-019$ \\
\hline 8.0 & 1e-019 \\
\hline 10.0 & $1.04 \mathrm{e}-019$ \\
\hline 12.0 & $1.09 \mathrm{e}-019$ \\
\hline 15.0 & $1.1 \mathrm{e}-019$ \\
\hline 17.0 & $1.07 \mathrm{e}-019$ \\
\hline 20.0 & $1.02 \mathrm{e}-019$ \\
\hline 25.0 & $9.5 \mathrm{e}-020$ \\
\hline 30.0 & $9 \mathrm{e}-020$ \\
\hline 50.0 & $8.6 \mathrm{e}-020$ \\
\hline 75.0 & $6.6 \mathrm{e}-020$ \\
\hline 100.0 & $5.8 \mathrm{e}-020$ \\
\hline 150.0 & $4.9 \mathrm{e}-020$ \\
\hline 200.0 & $4.2 \mathrm{e}-020$ \\
\hline 300.0 & $3.3 \mathrm{e}-020$ \\
\hline 500.0 & $2.44 \mathrm{e}-020$ \\
\hline 700.0 & $1.96 \mathrm{e}-020$ \\
\hline 1000.0 & $1.55 \mathrm{e}-020$ \\
\hline 1500.0 & $1.12 \mathrm{e}-020$ \\
\hline 2000.0 & $8.1 \mathrm{e}-021$ \\
\hline 3000.0 & $6.3 \mathrm{e}-021$ \\
\hline 5000.0 & $4 \mathrm{e}-021$ \\
\hline 7000.0 & $2.9 \mathrm{e}-021$ \\
\hline 10000.0 & $2.1 \mathrm{e}-021$ \\
\hline
\end{tabular}


Table B.2: Cross section data for $\mathrm{N}_{2}$ Excitation $\left(\Delta E^{e}=0.02 \mathrm{eV}\right)$

\begin{tabular}{|c|c|}
\hline Energy (eV) & Cross Section $\left.\mathbf{( m}^{2}\right)$ \\
\hline 0.0 & 0.0 \\
0.02 & 0.0 \\
0.03 & 0.0 \\
0.4 & 0.0 \\
0.8 & 0.0 \\
1.2 & $6 \mathrm{e}-022$ \\
1.6 & $1.8 \mathrm{e}-021$ \\
1.7 & $2.3 \mathrm{e}-021$ \\
1.8 & $4 \mathrm{e}-021$ \\
1.9 & $1.41 \mathrm{e}-020$ \\
2.0 & $5.13 \mathrm{e}-020$ \\
2.1 & $5.42 \mathrm{e}-020$ \\
2.2 & $5.14 \mathrm{e}-020$ \\
2.3 & $6.9 \mathrm{e}-020$ \\
2.4 & $6.04 \mathrm{e}-020$ \\
2.5 & $6.45 \mathrm{e}-020$ \\
2.6 & $5.1 \mathrm{e}-020$ \\
2.7 & $4.24 \mathrm{e}-020$ \\
2.8 & $3.75 \mathrm{e}-020$ \\
2.9 & $2.11 \mathrm{e}-020$ \\
3.0 & $2.32 \mathrm{e}-020$ \\
3.1 & $1.94 \mathrm{e}-020$ \\
3.2 & $1.4 \mathrm{e}-020$ \\
3.3 & $9.4 \mathrm{e}-021$ \\
3.6 & $3.8 \mathrm{e}-021$ \\
5.0 & 0.0 \\
20.0 & 0.0 \\
1000.0 & 0.0 \\
\hline \hline
\end{tabular}

Table B.3: Cross section data for $\mathrm{N}_{2}$ Excitation $\left(\Delta E^{e}=0.291 \mathrm{eV}\right)$

\begin{tabular}{|c|c|}
\hline Energy (eV) & Cross Section $\left(\mathbf{m}^{2}\right)$ \\
\hline 0.0 & 0.0 \\
0.291 & 0.0 \\
1.6 & 0.0 \\
1.65 & $2.7 \mathrm{e}-021$ \\
1.7 & $3.15 \mathrm{e}-021$ \\
1.8 & $5.4 \mathrm{e}-021$ \\
1.9 & $1.48 \mathrm{e}-020$ \\
2.0 & $4.8 \mathrm{e}-020$ \\
2.1 & $2.565 \mathrm{e}-020$ \\
2.2 & $1.2 \mathrm{e}-020$ \\
2.3 & $4.5 \mathrm{e}-020$ \\
2.4 & $2.76 \mathrm{e}-020$ \\
2.5 & $1.59 \mathrm{e}-020$ \\
2.6 & $3.15 \mathrm{e}-020$ \\
2.7 & $1.545 \mathrm{e}-020$ \\
2.75 & $6 \mathrm{e}-021$ \\
2.8 & $1.35 \mathrm{e}-020$ \\
2.9 & $5.25 \mathrm{e}-021$ \\
\hline \multicolumn{2}{|c|}{ Continued on next page } \\
\hline
\end{tabular}


Table B.3 - continued from previous page

\begin{tabular}{|c|c|}
\hline Energy (eV) & Cross Section $\left(\mathbf{m}^{2}\right)$ \\
\hline 3.0 & $8.7 \mathrm{e}-021$ \\
3.1 & $1.17 \mathrm{e}-020$ \\
3.2 & $8.55 \mathrm{e}-021$ \\
3.3 & $6.6 \mathrm{e}-021$ \\
3.4 & $6 \mathrm{e}-021$ \\
3.5 & $5.85 \mathrm{e}-021$ \\
3.6 & $5.7 \mathrm{e}-021$ \\
4.0 & 0.0 \\
100.0 & 0.0 \\
1000.0 & 0.0 \\
\hline \hline
\end{tabular}

Table B.4: Cross section data for $\mathrm{N}_{2}$ Excitation $\left(\Delta E^{e}=0.59 \mathrm{eV}\right)$

\begin{tabular}{|c|c|}
\hline Energy $(\mathbf{e V})$ & Cross Section $\left(\mathbf{m}^{2}\right)$ \\
\hline 0.0 & 0.0 \\
0.59 & 0.0 \\
1.7 & 0.0 \\
1.8 & $1.5 \mathrm{e}-022$ \\
1.9 & $6.3 \mathrm{e}-021$ \\
2.0 & $1.935 \mathrm{e}-020$ \\
2.1 & $3.3 \mathrm{e}-020$ \\
2.2 & $1.47 \mathrm{e}-020$ \\
2.3 & $5.4 \mathrm{e}-021$ \\
2.4 & $2.115 \mathrm{e}-020$ \\
2.5 & $3 \mathrm{e}-020$ \\
2.6 & $5.4 \mathrm{e}-021$ \\
2.7 & $1.05 \mathrm{e}-020$ \\
2.75 & $1.725 \mathrm{e}-020$ \\
2.8 & $1.275 \mathrm{e}-020$ \\
2.9 & $3.3 \mathrm{e}-021$ \\
3.0 & $9 \mathrm{e}-021$ \\
3.1 & $6.45 \mathrm{e}-021$ \\
3.2 & $3.75 \mathrm{e}-021$ \\
3.3 & $3.45 \mathrm{e}-021$ \\
3.4 & $3 \mathrm{e}-021$ \\
3.5 & $2.13 \mathrm{e}-021$ \\
3.6 & 0.0 \\
1000.0 & 0.0 \\
\hline \hline
\end{tabular}

Table B.5: Cross section data for $\mathrm{N}_{2}$ Excitation $\left(\Delta E^{e}=0.88 \mathrm{eV}\right)$

\begin{tabular}{|c|c|}
\hline Energy (eV) & Cross Section $\left.\mathbf{~}^{2}\right)$ \\
\hline 0.0 & 0.0 \\
0.88 & 0.0 \\
1.9 & 0.0 \\
2.0 & $9.6 \mathrm{e}-021$ \\
\hline \multicolumn{2}{|c|}{ Continued on next page } \\
\hline
\end{tabular}


Table B.5 - continued from previous page

\begin{tabular}{|c|c|}
\hline Energy (eV) & Cross Section $\left.\mathbf{( m}^{2}\right)$ \\
\hline 2.1 & $2.055 \mathrm{e}-020$ \\
2.2 & $2.7 \mathrm{e}-020$ \\
2.3 & $1.695 \mathrm{e}-020$ \\
2.4 & $7.5 \mathrm{e}-022$ \\
2.5 & $9.6 \mathrm{e}-021$ \\
2.6 & $1.47 \mathrm{e}-020$ \\
2.7 & $4.5 \mathrm{e}-021$ \\
2.75 & $9.6 \mathrm{e}-021$ \\
2.8 & $5.4 \mathrm{e}-021$ \\
2.9 & $8.55 \mathrm{e}-021$ \\
3.0 & $4.05 \mathrm{e}-021$ \\
3.1 & $2.82 \mathrm{e}-021$ \\
3.2 & $2.91 \mathrm{e}-021$ \\
3.3 & $6.15 \mathrm{e}-022$ \\
3.4 & 0.0 \\
1000.0 & 0.0 \\
\hline \hline
\end{tabular}

Table B.6: Cross section data for $\mathrm{N}_{2}$ Excitation $\left(\Delta E^{e}=1.17 \mathrm{eV}\right)$

\begin{tabular}{|c|c|}
\hline Energy $(\mathbf{e V})$ & Cross Section $\left(\mathbf{m}^{2}\right)$ \\
\hline 0.0 & 0.0 \\
1.17 & 0.0 \\
2.0 & 0.0 \\
2.1 & $2.025 \mathrm{e}-021$ \\
2.2 & $1.515 \mathrm{e}-020$ \\
2.3 & $2.385 \mathrm{e}-020$ \\
2.4 & $1.44 \mathrm{e}-020$ \\
2.5 & $5.55 \mathrm{e}-021$ \\
2.6 & $8.25 \mathrm{e}-022$ \\
2.7 & $1.2 \mathrm{e}-020$ \\
2.75 & $1.095 \mathrm{e}-020$ \\
2.8 & $6.75 \mathrm{e}-021$ \\
2.9 & $3 \mathrm{e}-022$ \\
3.0 & $3.3 \mathrm{e}-021$ \\
3.1 & $3.15 \mathrm{e}-021$ \\
3.2 & $6 \mathrm{e}-022$ \\
3.3 & 0.0 \\
1000.0 & 0.0 \\
\hline \hline
\end{tabular}

Table B.7: Cross section data for $\mathrm{N}_{2}$ Excitation $\left(\Delta E^{e}=1.47 \mathrm{eV}\right)$

\begin{tabular}{|c|c|}
\hline Energy (eV) & Cross Section $\left.\mathbf{~}^{2}\right)$ \\
\hline 0.0 & 0.0 \\
1.47 & 0.0 \\
2.1 & 0.0 \\
2.2 & $8.25 \mathrm{e}-021$ \\
\hline \multicolumn{2}{|c|}{ Continued on next page } \\
\hline
\end{tabular}


Table B.7 - continued from previous page

\begin{tabular}{|c|c|}
\hline Energy (eV) & Cross Section $\left(\mathbf{m}^{2}\right)$ \\
\hline 2.3 & $1.23 \mathrm{e}-020$ \\
2.4 & $1.53 \mathrm{e}-020$ \\
2.5 & $1.44 \mathrm{e}-020$ \\
2.6 & $3.45 \mathrm{e}-021$ \\
2.7 & $2.25 \mathrm{e}-022$ \\
2.75 & $3.45 \mathrm{e}-021$ \\
2.8 & $5.4 \mathrm{e}-021$ \\
2.9 & $6.6 \mathrm{e}-021$ \\
3.0 & $2.175 \mathrm{e}-021$ \\
3.1 & $1.05 \mathrm{e}-021$ \\
3.2 & $3.15 \mathrm{e}-021$ \\
3.3 & $1.035 \mathrm{e}-021$ \\
3.4 & 0.0 \\
1000.0 & 0.0 \\
\hline \hline
\end{tabular}

Table B.8: Cross section data for $\mathrm{N}_{2}$ Excitation $\left(\Delta E^{e}=1.76 \mathrm{eV}\right)$

\begin{tabular}{|c|c|}
\hline Energy $(\mathbf{e V})$ & Cross Section $\left(\mathbf{m}^{2}\right)$ \\
\hline 0.0 & 0.0 \\
1.76 & 0.0 \\
2.2 & 0.0 \\
2.3 & $6.3 \mathrm{e}-023$ \\
2.4 & $1.125 \mathrm{e}-020$ \\
2.5 & $1.74 \mathrm{e}-020$ \\
2.6 & $1.38 \mathrm{e}-020$ \\
2.7 & $7.8 \mathrm{e}-021$ \\
2.75 & $4.5 \mathrm{e}-021$ \\
2.8 & $3.15 \mathrm{e}-021$ \\
2.9 & $2.46 \mathrm{e}-021$ \\
3.0 & $4.8 \mathrm{e}-021$ \\
3.1 & $1.635 \mathrm{e}-021$ \\
3.2 & 0.0 \\
100.0 & 0.0 \\
1000.0 & 0.0 \\
\hline \hline
\end{tabular}

Table B.9: Cross section data for $\mathrm{N}_{2}$ Excitation $\left(\Delta E^{e}=2.06 \mathrm{eV}\right)$

\begin{tabular}{|c|c|}
\hline Energy (eV) & Cross Section $\left.\mathbf{( m}^{2}\right)$ \\
\hline 0.0 & 0.0 \\
2.06 & 0.0 \\
2.3 & 0.0 \\
2.4 & $1.26 \mathrm{e}-022$ \\
2.5 & $3.9 \mathrm{e}-021$ \\
2.6 & $6.6 \mathrm{e}-021$ \\
2.7 & $9.6 \mathrm{e}-021$ \\
2.75 & $7.95 \mathrm{e}-021$ \\
\hline \multicolumn{2}{|c}{ Continued on next page } \\
\hline
\end{tabular}


Table B.9 - continued from previous page

\begin{tabular}{|c|c|}
\hline Energy (eV) & Cross Section ${\left(\mathbf{m}^{2}\right)}^{2}$ \\
\hline 2.8 & $6 \mathrm{e}-021$ \\
2.9 & $1.8 \mathrm{e}-021$ \\
3.0 & $6.3 \mathrm{e}-023$ \\
3.1 & $1.92 \mathrm{e}-021$ \\
3.2 & $2.04 \mathrm{e}-021$ \\
3.3 & $7.8 \mathrm{e}-022$ \\
3.4 & $1.89 \mathrm{e}-022$ \\
3.5 & 0.0 \\
100.0 & 0.0 \\
1000.0 & 0.0 \\
\hline
\end{tabular}

Table B.10: Cross section data for $\mathrm{N}_{2}$ Excitation $\left(\Delta E^{e}=2.35 \mathrm{eV}\right)$

\begin{tabular}{|c|c|}
\hline Energy (eV) & Cross Section $\left(\mathbf{m}^{2}\right)$ \\
\hline 0.0 & 0.0 \\
2.35 & 0.0 \\
2.5 & 0.0 \\
2.6 & $1.89 \mathrm{e}-022$ \\
2.7 & $3.6 \mathrm{e}-021$ \\
2.75 & $3.6 \mathrm{e}-021$ \\
2.8 & $3.3 \mathrm{e}-021$ \\
2.9 & $3.45 \mathrm{e}-021$ \\
3.0 & $2.64 \mathrm{e}-021$ \\
3.1 & $3.75 \mathrm{e}-022$ \\
3.2 & $6.3 \mathrm{e}-023$ \\
3.3 & $1.545 \mathrm{e}-021$ \\
3.4 & $2.52 \mathrm{e}-022$ \\
3.5 & 0.0 \\
100.0 & 0.0 \\
1000.0 & 0.0 \\
\hline \hline
\end{tabular}

Table B.11: Cross section data for $\mathrm{N}_{2}$ Excitation $\left(\Delta E^{e}=6.17 \mathrm{eV}\right)$

\begin{tabular}{|c|c|}
\hline Energy (eV) & Cross Section $\left(\mathbf{m}^{2}\right)$ \\
\hline 0.0 & 0.0 \\
6.17 & 0.0 \\
7.0 & $3 \mathrm{e}-022$ \\
8.0 & $6.2 \mathrm{e}-022$ \\
9.0 & $9.4 \mathrm{e}-022$ \\
10.0 & $1.22 \mathrm{e}-021$ \\
11.0 & $1.48 \mathrm{e}-021$ \\
12.0 & $1.71 \mathrm{e}-021$ \\
13.0 & $1.89 \mathrm{e}-021$ \\
14.0 & $2.04 \mathrm{e}-021$ \\
15.0 & $2.15 \mathrm{e}-021$ \\
16.0 & $2.23 \mathrm{e}-021$ \\
\hline \multicolumn{2}{|c}{ Continued on next page } \\
\hline
\end{tabular}


Table B.11 - continued from previous page

\begin{tabular}{|c|c|}
\hline Energy (eV) & Cross Section $\left(\mathbf{m}^{2}\right)$ \\
\hline 17.0 & $2.25 \mathrm{e}-021$ \\
18.0 & $2.14 \mathrm{e}-021$ \\
19.0 & $1.99 \mathrm{e}-021$ \\
20.0 & $1.83 \mathrm{e}-021$ \\
22.0 & $1.55 \mathrm{e}-021$ \\
26.0 & $1.13 \mathrm{e}-021$ \\
30.0 & $8.7 \mathrm{e}-022$ \\
34.0 & $7.2 \mathrm{e}-022$ \\
40.0 & $5.8 \mathrm{e}-022$ \\
50.0 & $5 \mathrm{e}-022$ \\
70.0 & 0.0 \\
150.0 & 0.0 \\
1000.0 & 0.0 \\
\hline
\end{tabular}

Table B.12: Cross section data for $\mathrm{N}_{2}$ Excitation $\left(\Delta E^{e}=7.35 \mathrm{eV}\right)$

\begin{tabular}{|c|c|}
\hline Energy (eV) & Cross Section $\left.\mathbf{( m}^{2}\right)$ \\
\hline 0.0 & 0.0 \\
7.35 & 0.0 \\
8.0 & $5.4 \mathrm{e}-022$ \\
9.0 & $1.4 \mathrm{e}-021$ \\
10.0 & $2.25 \mathrm{e}-021$ \\
11.0 & $2.78 \mathrm{e}-021$ \\
12.0 & $2.99 \mathrm{e}-021$ \\
13.0 & $2.97 \mathrm{e}-021$ \\
14.0 & $2.71 \mathrm{e}-021$ \\
15.0 & $2.41 \mathrm{e}-021$ \\
16.0 & $2.16 \mathrm{e}-021$ \\
17.0 & $1.95 \mathrm{e}-021$ \\
18.0 & $1.79 \mathrm{e}-021$ \\
20.0 & $1.56 \mathrm{e}-021$ \\
22.0 & $1.42 \mathrm{e}-021$ \\
26.0 & $1.2 \mathrm{e}-021$ \\
30.0 & $1.01 \mathrm{e}-021$ \\
34.0 & $8.4 \mathrm{e}-022$ \\
40.0 & $6.4 \mathrm{e}-022$ \\
50.0 & $4 \mathrm{e}-022$ \\
70.0 & $1 \mathrm{e}-022$ \\
150.0 & 0.0 \\
500.0 & 0.0 \\
1000.0 & 0.0 \\
\hline \hline
\end{tabular}

Table B.13: Cross section data for $\mathrm{N}_{2}$ Excitation $\left(\Delta E^{e}=7.36 \mathrm{eV}\right)$

\begin{tabular}{|c|c|}
\hline Energy (eV) & Cross Section $\left.\mathbf{~ ( m}^{\mathbf{2}}\right)$ \\
\hline 0.0 & 0.0 \\
\hline \multicolumn{2}{|c|}{ Continued on next page } \\
\hline
\end{tabular}


Table B.13 - continued from previous page

\begin{tabular}{|c|c|}
\hline Energy (eV) & Cross Section $\left.\mathbf{( m}^{2}\right)$ \\
\hline 7.36 & 0.0 \\
8.0 & $2.7 \mathrm{e}-022$ \\
9.0 & $7.4 \mathrm{e}-022$ \\
10.0 & $1.2 \mathrm{e}-021$ \\
11.0 & $1.66 \mathrm{e}-021$ \\
12.0 & $2.13 \mathrm{e}-021$ \\
13.0 & $2.6 \mathrm{e}-021$ \\
14.0 & $3.06 \mathrm{e}-021$ \\
15.0 & $3.51 \mathrm{e}-021$ \\
16.0 & $3.8 \mathrm{e}-021$ \\
17.0 & $3.76 \mathrm{e}-021$ \\
18.0 & $3.5 \mathrm{e}-021$ \\
20.0 & $2.65 \mathrm{e}-021$ \\
22.0 & $1.97 \mathrm{e}-021$ \\
24.0 & $1.53 \mathrm{e}-021$ \\
26.0 & $1.26 \mathrm{e}-021$ \\
28.0 & $1.08 \mathrm{e}-021$ \\
30.0 & $9.4 \mathrm{e}-022$ \\
34.0 & $7.4 \mathrm{e}-022$ \\
40.0 & $5.2 \mathrm{e}-022$ \\
50.0 & $3 \mathrm{e}-022$ \\
70.0 & $1.5 \mathrm{e}-022$ \\
100.0 & $7 \mathrm{e}-023$ \\
150.0 & 0.0 \\
500.0 & 0.0 \\
1000.0 & 0.0 \\
\hline \hline
\end{tabular}

Table B.14: Cross section data for $\mathrm{N}_{2}$ Excitation $\left(\Delta E^{e}=8.16 \mathrm{eV}\right)$

\begin{tabular}{|c|c|}
\hline Energy (eV) & Cross Section $\left.\mathbf{( m}^{2}\right)$ \\
\hline 0.0 & 0.0 \\
8.16 & 0.0 \\
9.0 & $1.6 \mathrm{e}-022$ \\
10.0 & $3.5 \mathrm{e}-022$ \\
11.0 & $5.5 \mathrm{e}-022$ \\
12.0 & $7.4 \mathrm{e}-022$ \\
13.0 & $9.4 \mathrm{e}-022$ \\
14.0 & $1.13 \mathrm{e}-021$ \\
15.0 & $1.25 \mathrm{e}-021$ \\
16.0 & $1.14 \mathrm{e}-021$ \\
17.0 & $9.2 \mathrm{e}-022$ \\
18.0 & $7.3 \mathrm{e}-022$ \\
20.0 & $5.4 \mathrm{e}-022$ \\
22.0 & $4.7 \mathrm{e}-022$ \\
26.0 & $3.95 \mathrm{e}-022$ \\
30.0 & $3.37 \mathrm{e}-022$ \\
34.0 & $2.92 \mathrm{e}-022$ \\
40.0 & $2.45 \mathrm{e}-022$ \\
50.0 & $1.9 \mathrm{e}-022$ \\
70.0 & $1 \mathrm{e}-022$ \\
150.0 & 0.0 \\
\hline
\end{tabular}


Table B.14 - continued from previous page

\begin{tabular}{|c|c|}
\hline Energy (eV) & Cross Section $\left(\mathbf{m}^{2}\right)$ \\
\hline 500.0 & 0.0 \\
1000.0 & 0.0 \\
\hline \hline
\end{tabular}

Table B.15: Cross section data for $\mathrm{N}_{2}$ Excitation $\left(\Delta E^{e}=8.4 \mathrm{eV}\right)$

\begin{tabular}{|c|c|}
\hline Energy (eV) & Cross Section $\left(\mathbf{m}^{2}\right)$ \\
\hline 0.0 & 0.0 \\
8.4 & 0.0 \\
9.0 & $1 \mathrm{e}-022$ \\
11.0 & $4.5 \mathrm{e}-022$ \\
13.0 & $8 \mathrm{e}-022$ \\
14.0 & $9.6 \mathrm{e}-022$ \\
15.0 & $1.04 \mathrm{e}-021$ \\
16.0 & $8.5 \mathrm{e}-022$ \\
17.0 & $6.4 \mathrm{e}-022$ \\
18.0 & $5.2 \mathrm{e}-022$ \\
19.0 & $4.55 \mathrm{e}-022$ \\
20.0 & $4.1 \mathrm{e}-022$ \\
24.0 & $3 \mathrm{e}-022$ \\
30.0 & $2.3 \mathrm{e}-022$ \\
40.0 & $1.85 \mathrm{e}-022$ \\
50.0 & $1.8 \mathrm{e}-022$ \\
70.0 & $1.5 \mathrm{e}-022$ \\
150.0 & $1 \mathrm{e}-022$ \\
500.0 & 0.0 \\
1000.0 & 0.0 \\
\hline \hline
\end{tabular}

Table B.16: Cross section data for $\mathrm{N}_{2}$ Excitation $\left(\Delta E^{e}=8.55 \mathrm{eV}\right)$

\begin{tabular}{|c|c|}
\hline Energy (eV) & Cross Section $\left(\mathbf{m}^{2}\right)$ \\
\hline 0.0 & 0.0 \\
8.55 & 0.0 \\
9.0 & $1.9 \mathrm{e}-022$ \\
14.0 & $2.2 \mathrm{e}-021$ \\
15.0 & $2.56 \mathrm{e}-021$ \\
16.0 & $2.86 \mathrm{e}-021$ \\
17.0 & $3.02 \mathrm{e}-021$ \\
18.0 & $2.97 \mathrm{e}-021$ \\
19.0 & $2.87 \mathrm{e}-021$ \\
20.0 & $2.76 \mathrm{e}-021$ \\
24.0 & $2.42 \mathrm{e}-021$ \\
26.0 & $2.28 \mathrm{e}-021$ \\
30.0 & $2.04 \mathrm{e}-021$ \\
40.0 & $1.59 \mathrm{e}-021$ \\
50.0 & $1.27 \mathrm{e}-021$ \\
70.0 & $9 \mathrm{e}-022$ \\
\hline \multicolumn{2}{|c}{} \\
\hline
\end{tabular}


Table B.16 - continued from previous page

\begin{tabular}{|c|c|}
\hline Energy (eV) & Cross Section $\left(\mathbf{m}^{2}\right)$ \\
\hline 100.0 & $6 \mathrm{e}-022$ \\
150.0 & $4 \mathrm{e}-022$ \\
200.0 & $3 \mathrm{e}-022$ \\
250.0 & $2.4 \mathrm{e}-022$ \\
300.0 & $2 \mathrm{e}-022$ \\
500.0 & $1.2 \mathrm{e}-022$ \\
700.0 & $9 \mathrm{e}-023$ \\
1000.0 & $6 \mathrm{e}-023$ \\
\hline
\end{tabular}

Table B.17: Cross section data for $\mathrm{N}_{2}$ Excitation $\left(\Delta E^{e}=8.89 \mathrm{eV}\right)$

\begin{tabular}{|c|c|}
\hline Energy (eV) & Cross Section $\left(\mathbf{m}^{2}\right)$ \\
\hline 0.0 & 0.0 \\
8.89 & 0.0 \\
9.0 & $2 \mathrm{e}-023$ \\
10.0 & $3.9 \mathrm{e}-022$ \\
11.0 & $7.1 \mathrm{e}-022$ \\
12.0 & $9.9 \mathrm{e}-022$ \\
13.0 & $1.17 \mathrm{e}-021$ \\
14.0 & $1.15 \mathrm{e}-021$ \\
15.0 & $1 \mathrm{e}-021$ \\
16.0 & $8.1 \mathrm{e}-022$ \\
17.0 & $6.6 \mathrm{e}-022$ \\
18.0 & $5.6 \mathrm{e}-022$ \\
20.0 & $4.3 \mathrm{e}-022$ \\
22.0 & $3.6 \mathrm{e}-022$ \\
30.0 & $2.3 \mathrm{e}-022$ \\
38.0 & $1.4 \mathrm{e}-022$ \\
50.0 & $7 \mathrm{e}-023$ \\
150.0 & 0.0 \\
500.0 & 0.0 \\
1000.0 & 0.0 \\
\hline \hline
\end{tabular}

Table B.18: Cross section data for $\mathrm{N}_{2}$ Excitation $\left(\Delta E^{e}=11.03 \mathrm{eV}\right)$

\begin{tabular}{|c|c|}
\hline Energy (eV) & Cross Section $\left(\mathbf{m}^{2}\right)$ \\
\hline 0.0 & 0.0 \\
11.03 & 0.0 \\
12.0 & $1.46 \mathrm{e}-021$ \\
13.0 & $2.98 \mathrm{e}-021$ \\
14.0 & $4.43 \mathrm{e}-021$ \\
15.0 & $3.89 \mathrm{e}-021$ \\
16.0 & $2.84 \mathrm{e}-021$ \\
17.0 & $2.34 \mathrm{e}-021$ \\
18.0 & $2.02 \mathrm{e}-021$ \\
19.0 & $1.81 \mathrm{e}-021$ \\
\hline \multicolumn{2}{|c}{ Continued on next page } \\
\hline
\end{tabular}


Table B.18 - continued from previous page

\begin{tabular}{|c|c|}
\hline Energy (eV) & Cross Section $\left.\mathbf{( m}^{2}\right)$ \\
\hline 20.0 & $1.65 \mathrm{e}-021$ \\
22.0 & $1.39 \mathrm{e}-021$ \\
24.0 & $1.18 \mathrm{e}-021$ \\
26.0 & $1 \mathrm{e}-021$ \\
28.0 & $8.6 \mathrm{e}-022$ \\
30.0 & $7.4 \mathrm{e}-022$ \\
36.0 & $5.2 \mathrm{e}-022$ \\
40.0 & $4.2 \mathrm{e}-022$ \\
50.0 & $2.6 \mathrm{e}-022$ \\
70.0 & 0.0 \\
100.0 & 0.0 \\
150.0 & 0.0 \\
500.0 & 0.0 \\
1000.0 & 0.0 \\
\hline \hline
\end{tabular}

Table B.19: Cross section data for $\mathrm{N}_{2}$ Excitation $\left(\Delta E^{e}=11.88 \mathrm{eV}\right)$

\begin{tabular}{|c|c|}
\hline Energy (eV) & Cross Section $\left(\mathbf{m}^{2}\right)$ \\
\hline 0.0 & 0.0 \\
11.88 & 0.0 \\
12.0 & $5 \mathrm{e}-024$ \\
13.0 & $1 \mathrm{e}-023$ \\
14.0 & $2.1 \mathrm{e}-023$ \\
15.0 & $3 \mathrm{e}-023$ \\
16.0 & $4 \mathrm{e}-023$ \\
17.0 & $5 \mathrm{e}-023$ \\
19.0 & $6.2 \mathrm{e}-023$ \\
20.0 & $7 \mathrm{e}-023$ \\
22.0 & $7.8 \mathrm{e}-023$ \\
24.0 & $8 \mathrm{e}-023$ \\
26.0 & $8 \mathrm{e}-023$ \\
28.0 & $6.5 \mathrm{e}-023$ \\
30.0 & $5 \mathrm{e}-023$ \\
32.0 & $4 \mathrm{e}-023$ \\
40.0 & $1.8 \mathrm{e}-023$ \\
50.0 & $7 \mathrm{e}-024$ \\
150.0 & 0.0 \\
500.0 & 0.0 \\
1000.0 & 0.0 \\
\hline \hline
\end{tabular}

Table B.20: Cross section data for $\mathrm{N}_{2}$ Excitation $\left(\Delta E^{e}=12.25 \mathrm{eV}\right)$

\begin{tabular}{|c|c|}
\hline Energy (eV) & Cross Section $\left.\mathbf{~}^{2}\right)$ \\
\hline 0.0 & 0.0 \\
12.25 & 0.0 \\
13.0 & $8 \mathrm{e}-023$ \\
\hline \multicolumn{2}{|c}{ Continued on next page } \\
\hline
\end{tabular}


Table B.20 - continued from previous page

\begin{tabular}{|c|c|}
\hline Energy (eV) & Cross Section $\left(\mathbf{m}^{2}\right)$ \\
\hline 15.0 & $2.8 \mathrm{e}-022$ \\
16.0 & $3.7 \mathrm{e}-022$ \\
17.0 & $4.5 \mathrm{e}-022$ \\
18.0 & $5.2 \mathrm{e}-022$ \\
19.0 & $5.7 \mathrm{e}-022$ \\
20.0 & $5.8 \mathrm{e}-022$ \\
22.0 & $5.1 \mathrm{e}-022$ \\
24.0 & $4.1 \mathrm{e}-022$ \\
26.0 & $3.4 \mathrm{e}-022$ \\
30.0 & $2.3 \mathrm{e}-022$ \\
32.0 & $2 \mathrm{e}-023$ \\
36.0 & $1.7 \mathrm{e}-022$ \\
40.0 & $1.6 \mathrm{e}-022$ \\
50.0 & $1.43 \mathrm{e}-022$ \\
70.0 & $1 \mathrm{e}-022$ \\
100.0 & $7.5 \mathrm{e}-023$ \\
150.0 & $5.4 \mathrm{e}-023$ \\
1000.0 & $1.2 \mathrm{e}-023$ \\
\hline \hline
\end{tabular}

Table B.21: Cross section data for $\mathrm{N}_{2}$ Excitation $\left(\Delta E^{e}=13.0 \mathrm{eV}\right)$

\begin{tabular}{|c|c|}
\hline Energy (eV) & Cross Section $\left.\mathbf{( m}^{\mathbf{2}}\right)$ \\
\hline 0.0 & 0.0 \\
13.0 & 0.0 \\
14.0 & $8.1 \mathrm{e}-022$ \\
15.0 & $1.9 \mathrm{e}-021$ \\
16.0 & $2.5 \mathrm{e}-021$ \\
17.0 & $4.2 \mathrm{e}-021$ \\
18.0 & $5.2 \mathrm{e}-021$ \\
20.0 & $7.5 \mathrm{e}-021$ \\
22.0 & $9.6 \mathrm{e}-021$ \\
25.0 & $1.19 \mathrm{e}-020$ \\
30.0 & $1.48 \mathrm{e}-020$ \\
40.0 & $1.65 \mathrm{e}-020$ \\
60.0 & $1.76 \mathrm{e}-020$ \\
80.0 & $1.68 \mathrm{e}-020$ \\
100.0 & $1.58 \mathrm{e}-020$ \\
150.0 & $1.33 \mathrm{e}-020$ \\
1000.0 & $5.3 \mathrm{e}-021$ \\
\hline \hline
\end{tabular}

Table B.22: Cross section data for $\mathrm{N}_{2}$ Ionization $\left(\Delta E^{e}=15.6 \mathrm{eV}\right)$

\begin{tabular}{|c|c|}
\hline Energy (eV) & Cross Section $\left.\mathbf{( m}^{2}\right)$ \\
\hline 16.0 & $2.1 \mathrm{e}-022$ \\
16.5 & $4.6 \mathrm{e}-022$ \\
17.0 & $7.1 \mathrm{e}-022$ \\
\hline \multicolumn{2}{|c}{ Continued on next page } \\
\hline
\end{tabular}


Table B.22 - continued from previous page

\begin{tabular}{|c|c|}
\hline Energy (eV) & Cross Section $\left(\mathbf{m}^{2}\right)$ \\
\hline 17.5 & $9.8 \mathrm{e}-022$ \\
18.0 & $1.29 \mathrm{e}-021$ \\
18.5 & $1.63 \mathrm{e}-021$ \\
19.0 & $1.98 \mathrm{e}-021$ \\
19.5 & $2.29 \mathrm{e}-021$ \\
20.0 & $2.69 \mathrm{e}-021$ \\
21.0 & $3.42 \mathrm{e}-021$ \\
22.0 & $4.16 \mathrm{e}-021$ \\
23.0 & $4.9 \mathrm{e}-021$ \\
25.0 & $6.37 \mathrm{e}-021$ \\
30.0 & $1.03 \mathrm{e}-020$ \\
34.0 & $1.26 \mathrm{e}-020$ \\
45.0 & $1.77 \mathrm{e}-020$ \\
60.0 & $2.17 \mathrm{e}-020$ \\
75.0 & $2.38 \mathrm{e}-020$ \\
100.0 & $2.52 \mathrm{e}-020$ \\
150.0 & $2.44 \mathrm{e}-020$ \\
200.0 & $2.26 \mathrm{e}-020$ \\
300.0 & $1.91 \mathrm{e}-020$ \\
500.0 & $1.45 \mathrm{e}-020$ \\
700.0 & $1.16 \mathrm{e}-020$ \\
1000.0 & $9.2 \mathrm{e}-021$ \\
1500.0 & $8 \mathrm{e}-021$ \\
\hline \hline
\end{tabular}

Table B.23: Cross section data for $\mathrm{O}_{2}$ Elastic $\left(\Delta E^{e}=1.7 e-005 \mathrm{eV}\right)$

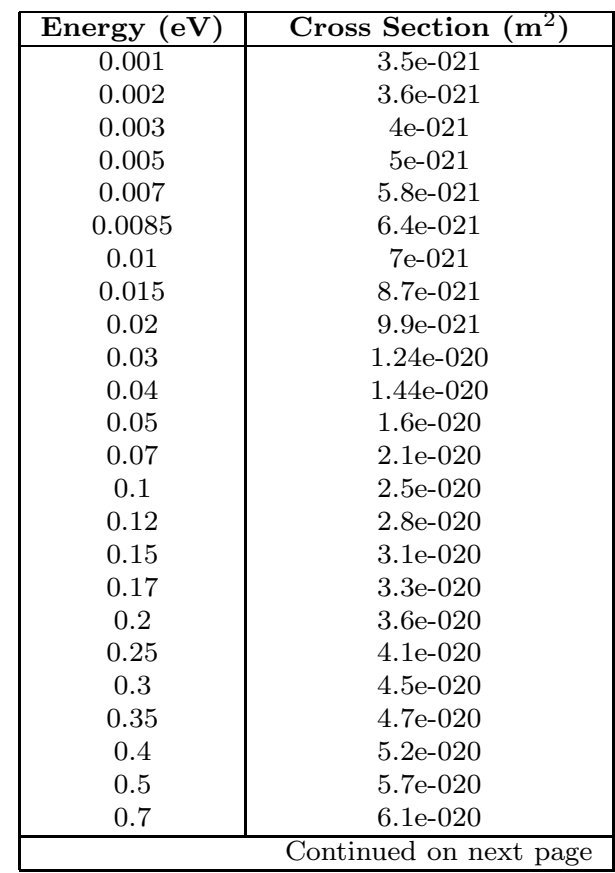


Table B.23 - continued from previous page

\begin{tabular}{|c|c|}
\hline Energy (eV) & Cross Section $\left(\mathrm{m}^{2}\right)$ \\
\hline 1.0 & $7.2 \mathrm{e}-020$ \\
\hline 1.2 & $7.9 \mathrm{e}-020$ \\
\hline 1.3 & $7.9 \mathrm{e}-020$ \\
\hline 1.5 & $7.6 \mathrm{e}-020$ \\
\hline 1.7 & $7.3 \mathrm{e}-020$ \\
\hline 1.9 & $6.9 \mathrm{e}-020$ \\
\hline 2.1 & $6.6 \mathrm{e}-020$ \\
\hline 2.2 & $6.5 \mathrm{e}-020$ \\
\hline 2.5 & $6.1 \mathrm{e}-020$ \\
\hline 2.8 & $5.8 \mathrm{e}-020$ \\
\hline 3.0 & $5.7 \mathrm{e}-020$ \\
\hline 3.3 & $5.5 \mathrm{e}-020$ \\
\hline 3.6 & $5.45 \mathrm{e}-020$ \\
\hline 4.0 & $5.5 \mathrm{e}-020$ \\
\hline 4.5 & $5.55 \mathrm{e}-020$ \\
\hline 5.0 & $5.6 \mathrm{e}-020$ \\
\hline 6.0 & $6 e-020$ \\
\hline 7.0 & $6.6 \mathrm{e}-020$ \\
\hline 8.0 & $7.1 \mathrm{e}-020$ \\
\hline 10.0 & $8 e-020$ \\
\hline 12.0 & $8.5 \mathrm{e}-020$ \\
\hline 15.0 & $8.8 \mathrm{e}-020$ \\
\hline 17.0 & $8.7 \mathrm{e}-020$ \\
\hline 20.0 & $8.6 \mathrm{e}-020$ \\
\hline 25.0 & $8.2 \mathrm{e}-020$ \\
\hline 30.0 & $8 \mathrm{e}-020$ \\
\hline 50.0 & $7.7 \mathrm{e}-020$ \\
\hline 75.0 & $6.8 \mathrm{e}-020$ \\
\hline 100.0 & $6.5 \mathrm{e}-020$ \\
\hline 150.0 & $6.7 \mathrm{e}-020$ \\
\hline 200.0 & $6 e-020$ \\
\hline 300.0 & $4.9 \mathrm{e}-020$ \\
\hline 500.0 & $3.6 \mathrm{e}-020$ \\
\hline 700.0 & $2.9 \mathrm{e}-020$ \\
\hline 1000.0 & $2.12 \mathrm{e}-020$ \\
\hline 1500.0 & $1.48 \mathrm{e}-020$ \\
\hline 2000.0 & $1.14 \mathrm{e}-020$ \\
\hline 3000.0 & $7.9 \mathrm{e}-021$ \\
\hline 5000.0 & $5.1 \mathrm{e}-021$ \\
\hline 7000.0 & $3.8 \mathrm{e}-021$ \\
\hline 10000.0 & $2.8 \mathrm{e}-021$ \\
\hline
\end{tabular}

Table B.24: Cross section data for $\mathrm{O}_{2}$ Excitation $\left(\Delta E^{e}=0.02 \mathrm{eV}\right)$

\begin{tabular}{|c|c|}
\hline Energy (eV) & Cross Section $\left.\mathbf{( m}^{2}\right)$ \\
\hline 0.0 & 0.0 \\
0.0067 & 0.0 \\
0.07 & 0.0 \\
0.08 & $5.4 \mathrm{e}-023$ \\
0.1 & 0.0 \\
0.2 & 0.0 \\
\hline \multicolumn{2}{|c}{ Continued on next page } \\
\hline
\end{tabular}


Table B.24 - continued from previous page

\begin{tabular}{|c|c|}
\hline Energy (eV) & Cross Section $\left(\mathrm{m}^{2}\right)$ \\
\hline 0.21 & $2.16 \mathrm{e}-022$ \\
\hline 0.22 & 0.0 \\
\hline 0.32 & 0.0 \\
\hline 0.33 & $3.84 \mathrm{e}-022$ \\
\hline 0.35 & 0.0 \\
\hline 0.44 & 0.0 \\
\hline 0.45 & $5.4 \mathrm{e}-022$ \\
\hline 0.47 & 0.0 \\
\hline 0.56 & 0.0 \\
\hline 0.57 & $6.72 \mathrm{e}-022$ \\
\hline 0.59 & 0.0 \\
\hline 0.68 & 0.0 \\
\hline 0.69 & $8.04 \mathrm{e}-022$ \\
\hline 0.71 & 0.0 \\
\hline 0.79 & 0.0 \\
\hline 0.8 & $9.36 \mathrm{e}-022$ \\
\hline 0.81 & 0.0 \\
\hline 0.9 & 0.0 \\
\hline 0.91 & $8.4 \mathrm{e}-022$ \\
\hline 0.93 & 0.0 \\
\hline 1.02 & 0.0 \\
\hline 1.03 & $7.2 \mathrm{e}-022$ \\
\hline 1.05 & 0.0 \\
\hline 1.13 & 0.0 \\
\hline 1.14 & $4.68 \mathrm{e}-022$ \\
\hline 1.16 & 0.0 \\
\hline 1.23 & $6 e-022$ \\
\hline 1.26 & 0.0 \\
\hline 1.34 & 0.0 \\
\hline 1.35 & $3.6 \mathrm{e}-022$ \\
\hline 1.37 & 0.0 \\
\hline 1.44 & 0.0 \\
\hline 1.45 & $2.4 \mathrm{e}-022$ \\
\hline 1.47 & 0.0 \\
\hline 1.54 & 0.0 \\
\hline 1.55 & $1.2 \mathrm{e}-022$ \\
\hline 1.57 & 0.0 \\
\hline 1.64 & 0.0 \\
\hline 1.65 & $4.8 \mathrm{e}-023$ \\
\hline 1.67 & 0.0 \\
\hline 1000.0 & 0.0 \\
\hline
\end{tabular}

Table B.25: Cross section data for $\mathrm{O}_{2}$ Excitation $\left(\Delta E^{e}=0.193 \mathrm{eV}\right)$

\begin{tabular}{|c|c|}
\hline Energy (eV) & Cross Section $\left.\mathbf{( m}^{2}\right)$ \\
\hline 0.0 & 0.0 \\
0.193 & 0.0 \\
0.2 & $7.5 \mathrm{e}-022$ \\
0.21 & $7.5 \mathrm{e}-022$ \\
0.23 & 0.0 \\
0.32 & 0.0 \\
\hline \multicolumn{2}{|c}{ Continued on next page } \\
\hline
\end{tabular}


Table B.25 - continued from previous page

\begin{tabular}{|c|c|}
\hline Energy (eV) & Cross Section $\left(\mathrm{m}^{2}\right)$ \\
\hline 0.33 & $3 \mathrm{e}-021$ \\
\hline 0.35 & 0.0 \\
\hline 0.44 & 0.0 \\
\hline 0.45 & $1.41 \mathrm{e}-020$ \\
\hline 0.47 & 0.0 \\
\hline 0.56 & 0.0 \\
\hline 0.57 & $1.82 \mathrm{e}-020$ \\
\hline 0.59 & 0.0 \\
\hline 0.68 & 0.0 \\
\hline 0.69 & $2.07 \mathrm{e}-020$ \\
\hline 0.71 & 0.0 \\
\hline 0.79 & 0.0 \\
\hline 0.8 & $1.78 \mathrm{e}-020$ \\
\hline 0.82 & 0.0 \\
\hline 0.9 & 0.0 \\
\hline 0.91 & $1.5 \mathrm{e}-020$ \\
\hline 0.93 & 0.0 \\
\hline 1.02 & 0.0 \\
\hline 1.03 & $1.01 \mathrm{e}-020$ \\
\hline 1.05 & 0.0 \\
\hline 1.13 & 0.0 \\
\hline 1.14 & $7.61 \mathrm{e}-021$ \\
\hline 1.16 & 0.0 \\
\hline 1.23 & 0.0 \\
\hline 1.24 & $5.42 \mathrm{e}-021$ \\
\hline 1.26 & 0.0 \\
\hline 1.34 & 0.0 \\
\hline 1.35 & $3.11 \mathrm{e}-021$ \\
\hline 1.37 & 0.0 \\
\hline 1.44 & 0.0 \\
\hline 1.45 & $2.33 \mathrm{e}-021$ \\
\hline 1.47 & 0.0 \\
\hline 1.54 & 0.0 \\
\hline 1.55 & $1.21 \mathrm{e}-021$ \\
\hline 1.57 & 0.0 \\
\hline 1.63 & 0.0 \\
\hline 1.65 & $7.63 e-022$ \\
\hline 1.67 & 0.0 \\
\hline 3.5 & 0.0 \\
\hline 4.0 & $3.2 \mathrm{e}-023$ \\
\hline 5.0 & $9.76 \mathrm{e}-022$ \\
\hline 6.0 & $2.09 \mathrm{e}-021$ \\
\hline 7.0 & $4.92 \mathrm{e}-021$ \\
\hline 8.0 & $6.66 \mathrm{e}-021$ \\
\hline 9.0 & $7.71 \mathrm{e}-021$ \\
\hline 10.0 & $7.54 \mathrm{e}-021$ \\
\hline 11.0 & $6.01 \mathrm{e}-021$ \\
\hline 12.0 & $4.78 \mathrm{e}-021$ \\
\hline 13.0 & $3.29 \mathrm{e}-021$ \\
\hline 14.0 & $1.95 \mathrm{e}-021$ \\
\hline 15.0 & $8.65 \mathrm{e}-022$ \\
\hline 20.0 & $1.99 \mathrm{e}-022$ \\
\hline 45.0 & 0.0 \\
\hline 1000.0 & 0.0 \\
\hline
\end{tabular}


Table B.26: Cross section data for $\mathrm{O}_{2}$ Excitation $\left(\Delta E^{e}=0.98 \mathrm{eV}\right)$

\begin{tabular}{|c|c|}
\hline Energy (eV) & Cross Section $\left(\mathbf{m}^{2}\right)$ \\
\hline 0.98 & 0.0 \\
1.5 & $5.8 \mathrm{e}-023$ \\
2.0 & $1.53 \mathrm{e}-022$ \\
3.0 & $3.8 \mathrm{e}-022$ \\
3.5 & $4.9 \mathrm{e}-022$ \\
4.0 & $5.7 \mathrm{e}-022$ \\
4.5 & $6.7 \mathrm{e}-022$ \\
5.0 & $7.4 \mathrm{e}-022$ \\
5.62 & $8.25 \mathrm{e}-022$ \\
5.91 & $8.62 \mathrm{e}-022$ \\
6.19 & $8.8 \mathrm{e}-022$ \\
6.53 & $9.08 \mathrm{e}-022$ \\
6.99 & $9.14 \mathrm{e}-022$ \\
7.61 & $8.91 \mathrm{e}-022$ \\
7.89 & $8.63 \mathrm{e}-022$ \\
8.96 & $7.68 \mathrm{e}-022$ \\
10.04 & $6.79 \mathrm{e}-022$ \\
13.0 & $5.27 \mathrm{e}-022$ \\
15.1 & $4.55 \mathrm{e}-022$ \\
17.5 & $3.87 \mathrm{e}-022$ \\
20.5 & $3.24 \mathrm{e}-022$ \\
24.9 & $2.56 \mathrm{e}-022$ \\
30.9 & $1.96 \mathrm{e}-022$ \\
41.0 & $1.37 \mathrm{e}-022$ \\
45.0 & $1.2 \mathrm{e}-022$ \\
1000.0 & 0.0 \\
\hline \hline
\end{tabular}

Table B.27: Cross section data for $\mathrm{O}_{2}$ Excitation $\left(\Delta E^{e}=1.63 \mathrm{eV}\right)$

\begin{tabular}{|c|c|}
\hline Energy (eV) & Cross Section $\left(\mathbf{m}^{2}\right)$ \\
\hline 0.0 & 0.0 \\
1.63 & 0.0 \\
2.0 & $2.6 \mathrm{e}-023$ \\
3.0 & $9.7 \mathrm{e}-023$ \\
3.5 & $1.33 \mathrm{e}-022$ \\
4.0 & $1.49 \mathrm{e}-022$ \\
4.5 & $1.7 \mathrm{e}-022$ \\
5.0 & $1.82 \mathrm{e}-022$ \\
5.69 & $1.94 \mathrm{e}-022$ \\
6.54 & $1.94 \mathrm{e}-022$ \\
7.34 & $1.91 \mathrm{e}-022$ \\
8.41 & $1.83 \mathrm{e}-022$ \\
9.26 & $1.74 \mathrm{e}-022$ \\
10.0 & $1.6 \mathrm{e}-022$ \\
13.0 & $1.31 \mathrm{e}-022$ \\
14.9 & $1.17 \mathrm{e}-022$ \\
17.0 & $1.03 \mathrm{e}-022$ \\
20.7 & $8.6 \mathrm{e}-023$ \\
22.5 & $8 \mathrm{e}-023$ \\
24.5 & $7.2 \mathrm{e}-023$ \\
\hline \multicolumn{2}{|c|}{ Continued on next page } \\
\hline
\end{tabular}


Table B.27 - continued from previous page

\begin{tabular}{|c|c|}
\hline Energy (eV) & Cross Section $\left.\mathbf{( m}^{2}\right)$ \\
\hline 28.0 & $6.1 \mathrm{e}-023$ \\
35.0 & $4.7 \mathrm{e}-023$ \\
41.9 & $3.4 \mathrm{e}-023$ \\
45.1 & $3.1 \mathrm{e}-023$ \\
1000.0 & 0.0 \\
\hline
\end{tabular}

Table B.28: Cross section data for $\mathrm{O}_{2}$ Excitation $\left(\Delta E^{e}=4.5 \mathrm{eV}\right)$

\begin{tabular}{|c|c|}
\hline Energy (eV) & Cross Section $\left.\mathbf{( m}^{\mathbf{2}}\right)$ \\
\hline 0.0 & 0.0 \\
4.5 & 0.0 \\
4.68 & $1.5 \mathrm{e}-022$ \\
4.72 & $3 \mathrm{e}-022$ \\
6.21 & $1.63 \mathrm{e}-021$ \\
6.37 & $1.74 \mathrm{e}-021$ \\
6.6 & $1.77 \mathrm{e}-021$ \\
6.84 & $1.67 \mathrm{e}-021$ \\
7.12 & $1.55 \mathrm{e}-021$ \\
7.39 & $1.41 \mathrm{e}-021$ \\
9.91 & $4.2 \mathrm{e}-022$ \\
10.42 & $3.1 \mathrm{e}-022$ \\
11.48 & $1 \mathrm{e}-023$ \\
1000.0 & 0.0 \\
\hline \hline
\end{tabular}

Table B.29: Cross section data for $\mathrm{O}_{2}$ Excitation $\left(\Delta E^{e}=5.58 \mathrm{eV}\right)$

\begin{tabular}{|c|c|}
\hline Energy (eV) & Cross Section $\left.\mathbf{( m}^{2}\right)$ \\
\hline 0.0 & 0.0 \\
5.58 & 0.0 \\
6.17 & $6 \mathrm{e}-023$ \\
6.68 & $1.4 \mathrm{e}-022$ \\
7.46 & $3 \mathrm{e}-022$ \\
11.51 & $1.58 \mathrm{e}-021$ \\
12.1 & $1.63 \mathrm{e}-021$ \\
12.57 & $1.6 \mathrm{e}-021$ \\
19.64 & $9.4 \mathrm{e}-022$ \\
20.82 & $8.9 \mathrm{e}-022$ \\
25.26 & $6.6 \mathrm{e}-022$ \\
25.89 & $6.4 \mathrm{e}-022$ \\
38.65 & $3.4 \mathrm{e}-022$ \\
45.64 & $2.1 \mathrm{e}-022$ \\
47.65 & $1.5 \mathrm{e}-022$ \\
1000.0 & 0.0 \\
\hline
\end{tabular}


Table B.30: Cross section data for $\mathrm{O}_{2}$ Excitation $\left(\Delta E^{e}=8.4 \mathrm{eV}\right)$

\begin{tabular}{|c|c|}
\hline Energy (eV) & Cross Section $\left(\mathbf{m}^{2}\right)$ \\
\hline 0.0 & 0.0 \\
8.4 & 0.0 \\
9.4 & $1 \mathrm{e}-020$ \\
30.0 & $1.2 \mathrm{e}-020$ \\
50.0 & $1.17 \mathrm{e}-020$ \\
100.0 & $9.4 \mathrm{e}-021$ \\
1000.0 & $9 \mathrm{e}-023$ \\
\hline \hline
\end{tabular}

Table B.31: Cross section data for $\mathrm{O}_{2}$ Attachment $\left(\Delta E^{e}=3.6 \mathrm{eV}\right)$

\begin{tabular}{|c|c|}
\hline Energy (eV) & Cross Section $\left(\mathbf{m}^{2}\right)$ \\
\hline 0.0 & 0.0 \\
4.2 & 0.0 \\
4.6 & $7 \mathrm{e}-024$ \\
5.0 & $2.2 \mathrm{e}-023$ \\
5.5 & $6.3 \mathrm{e}-023$ \\
6.0 & $1.14 \mathrm{e}-022$ \\
6.3 & $1.36 \mathrm{e}-022$ \\
6.5 & $1.41 \mathrm{e}-022$ \\
6.7 & $1.37 \mathrm{e}-022$ \\
7.0 & $1.22 \mathrm{e}-022$ \\
7.5 & $8.2 \mathrm{e}-023$ \\
8.0 & $4.5 \mathrm{e}-023$ \\
8.5 & $2 \mathrm{e}-023$ \\
9.0 & $9 \mathrm{e}-024$ \\
9.5 & $5 \mathrm{e}-024$ \\
9.9 & $4 \mathrm{e}-024$ \\
10.0 & $1 \mathrm{e}-024$ \\
100.0 & 0.0 \\
150.0 & 0.0 \\
1000.0 & 0.0 \\
\hline \hline
\end{tabular}

Table B.32: Cross section data for $\mathrm{O}_{2}$ Ionization $\left(\Delta E^{e}=12.07 \mathrm{eV}\right)$

\begin{tabular}{|c|c|}
\hline Energy (eV) & Cross Section $\left(\mathbf{m}^{2}\right)$ \\
\hline 0.0 & 0.0 \\
12.07 & 0.0 \\
13.0 & $2.5 \mathrm{e}-022$ \\
18.0 & $2.5 \mathrm{e}-021$ \\
28.0 & $1.024 \mathrm{e}-020$ \\
38.0 & $1.775 \mathrm{e}-020$ \\
48.0 & $2.07 \mathrm{e}-020$ \\
58.0 & $2.41 \mathrm{e}-020$ \\
68.0 & $2.61 \mathrm{e}-020$ \\
78.0 & $2.81 \mathrm{e}-020$ \\
\hline \multicolumn{2}{|c}{} \\
\hline
\end{tabular}


Table B.32 - continued from previous page

\begin{tabular}{|c|c|}
\hline Energy (eV) & Cross Section ${\left(\mathbf{m}^{2}\right)}^{2}$. $2.84 \mathrm{e}-020$ \\
88.0 & $2.9 \mathrm{e}-020$ \\
100.0 & $2.7 \mathrm{e}-020$ \\
150.0 & 0.0 \\
1000.0 & 0 \\
\hline
\end{tabular}




\section{Bibliography}

[1] M. A. Lieberman and A. J. Lichtenberg. Principles of Plasma Discharges and Materials Processing. Wiley-Interscience, 2nd edition, 2005.

[2] U. Kogelschatz. Dielectric-Barrier discharges: Their history, discharge physics, and industrial applications. Plasma Chemistry and Plasma Processing, 23(1):1-46, 2003.

[3] A. Bogaerts, E. Neyts, R. Gijbels, and J. van der Mullen. Gas discharge plasmas and their applications. Spectrochimica Acta Part B: Atomic Spectroscopy, 57(4):609-658, April 2002.

[4] V. E. Semenov, V. G. Bondarenko, V. B. Gildenburg, V. M. Gubchenko, and A. I. Smirnov. Weakly ionized plasmas in aerospace applications. Plasma Physics and Controlled Fusion, 44(12B):B293-B305, 2002.

[5] P. Bletzinger, B. N. Ganguly, D. Van Wie, and A. Garscadden. Plasmas in high speed aerodynamics. Journal of Physics D: Applied Physics, 38(4):R33-R57, 2005.

[6] E. Moreau. Airflow control by non-thermal plasma actuators. Journal of Physics D: Applied Physics, 40(3):605-636, 2007.

[7] E.M. Braun, F.K. Lu, and D.R. Wilson. Experimental research in aerodynamic control with electric and electromagnetic fields. Progress in 
Aerospace Sciences, 45(1-3):30-49, 2009.

[8] R. L. Kimmel, J. R. Hayes, J. A. Menart, and J. S. Shang, Effect of surface plasma discharges on boundary layers at Mach 5, AIAA Paper 2004-0509, 2004.

[9] S. Leonov, V. Bityurin, K. Savelkin, and D. Yarantsev, Effect of electrical discharge on separation processes and shocks position in supersonic airflow, AIAA Paper 2002-0355.

[10] S. Leonov and D. Yarantsev. Near-Surface electrical discharge in supersonic airflow: Properties and flow control. Journal of Propulsion and Power, 24(6):1168-1181, 2008.

[11] R. L. Kimmel, J. Hayes, J. Menart, and J. S. Shang. Effect of magnetic fields on surface plasma discharges at Mach 5. Journal of Spacecraft and Rockets, 43(6):1340-1346, 2006.

[12] J. S. Shang, S. T. Surzhikov, R. Kimmel, D. Gaitonde, J. Menart, and J. Hayes, Plasma actuators for hypersonic flow control, AIAA Paper 20050562, 2005.

[13] J. S. Shang, R. Kimmel, J. Menart, and S. T. Surzhikov. Hypersonic flow control using surface plasma actuator. Journal of Propulsion and Power, 24(5):923-934, 2008.

[14] J. Shin, V. Narayanaswamy, L. L. Raja, and N. T. Clemens. Characterization of a DC glow discharge plasma actuator in supersonic flow. AIAA Journal, 45:1596-1605, 2007. 
[15] E. Menier, L. Leger, E. Depussay, V. Lago, and G. Artana. Effect of a dc discharge on the supersonic rarefied air flow over a flat plate. Journal of Physics D: Applied Physics, 40(3):695-701, 2007.

[16] D. B. Graves and K. F. Jensen. A continuum model of DC and RF discharges. IEEE Transactions on Plasma Science, 14(2):78-91, 1986.

[17] J. P. Boeuf. A two-dimensional model of dc glow discharges. Journal of Applied Physics, 63:1342-1348, 1988.

[18] S. T Surzhikov and J. S. Shang. Two-component plasma model for twodimensional glow discharge in magnetic field. Journal of Computational Physics, 199(2):437-464, 2004.

[19] M. Meyyappan and J. P. Kreskovsky. Glow discharge simulation through solutions to the moments of the boltzmann transport equation. Journal of Applied Physics, 68:1506, 1990.

[20] M. H. Wilcoxson and V. I. Manousiouthakis. Well-posedness of continuum models for weakly ionized plasmas. IEEE Transactions on Plasma Science, 21(2):213-222, 1993.

[21] J. P. Boeuf and L. C. Pitchford. Two-dimensional model of a capacitively coupled rf discharge and comparisons with experiments in the Gaseous Electronics Conference reference reactor. Physical Review E, 51(2):1376, February 1995.

[22] L. J. Overzet and M. B. Hopkins. Comparison of electron-density measurements made using a langmuir probe and microwave interferometer in 
the Gaseous Electronics Conference reference reactor. Journal of Applied Physics, 74(7):4323-4330, October 1993.

[23] J. D. Bukowski, D. B. Graves, and P. Vitello. Two-dimensional fluid model of an inductively coupled plasma with comparison to experimental spatial profiles. Journal of Applied Physics, 80(5):2614-2623, 1996.

[24] A. Bogaerts and R. Gijbels. Modeling of argon direct current glow discharges and comparison with experiment: how good is the agreement? Journal of Analytical Atomic Spectrometry, 13(9):945-953, 1998.

[25] I. A. Kossyi, A. Y. Kostinsky, A. A. Matveyev, and V. P. Silakov. Kinetic scheme of the non-equilibrium discharge in nitrogen-oxygen mixtures. Plasma Sources Science and Technology, 1(3):207-220, 1992.

[26] J. Nahorny, C. M. Ferreira, B. Gordiets, D. Pagnon, M. Touzeau, and M. Vialle. Experimental and theoretical investigation of a N2-O2 DC flowing glow discharge. Journal of Physics D Applied Physics, 28:738747, April 1995.

[27] B. F. Gordiets, C. M. Ferreira, V. L. Guerra, J. M. A. H. Loureiro, J. Nahorny, D. Pagnon, M. Touzeau, and M. Vialle. Kinetic model of a lowpressure N2-O2 flowing glow discharge. IEEE Transactions on Plasma Science, 23(4):750-768, 1995.

[28] M. Castillo, I. Mendez, A.M. Islyaikin, V.J. Herrero, and I. Tanarro. Low-Pressure DC air plasmas. investigation of neutral and ion chemistry. Journal of Physical Chemistry A, 109(28):6255-6263, July 2005. 
[29] S. V. Pancheshnyi and A. Y. Starikovskii. Two-dimensional numerical modelling of the cathode-directed streamer development in a long gap at high voltage. Journal of Physics D: Applied Physics, 36(21):2683-2691, 2003.

[30] S. Pancheshnyi, M. Nudnova, and A. Starikovskii. Development of a cathode-directed streamer discharge in air at different pressures: Experiment and comparison with direct numerical simulation. Physical Review E, 71(1):16407, 2005.

[31] K. P. Singh and S. Roy. Modeling plasma actuators with air chemistry for effective flow control. Journal of Applied Physics, 101(12):123308-8, June 2007.

[32] J. Poggie, Numerical exploration of flow control with glow discharges, AIAA Paper 2004-2658, 2004.

[33] J. S. Shang, P. G. Huang, H. Yan, and S. T. Surzhikov. Computational electrodynamic simulation of direct current discharge. Journal of Applied Physics, 105(2):023303, 2009.

[34] T. Deconinck, S. Mahadevan, and L. L. Raja. Simulation of direct-current surface plasma discharge phenomena in high-speed flow actuation. IEEE Transactions on Plasma Science, 35(5):1301-1311, Oct. 2007.

[35] F. F. Young and C. H. Wu. A fully-vectorized code for nonequilibrium RF glow discharge modeling and its parallel processing on a cray X-MP. 
In Proceedings of the 1992 ACM/IEEE conference on Supercomputing, pages 424-432, 1992.

[36] C.-T. Hung, M.-H. Hu, J.-S. Wu, and F.-N. Hwang. A new paradigm for solving plasma fluid modeling equations. Computer Physics Communications, 177(1-2):138 - 139, 2007.

[37] S. Balay, K. Buschelman, W. D. Gropp, D. Kaushik, M. G. Knepley, L. C. McInnes, B. F. Smith, and H. Zhang. PETSc web page. http://www.mcs.anl.gov/petsc.

[38] A. V. Likhanskii, V. V. Semak, M. N. Shneider, D. F. Opaits, R. B. Miles, and S. O. Macheret, Multiprocessor modeling of DBD plasma actuators, AIAA Paper 2008-4284, 2008.

[39] T. Deconinck, S. Mahadevan, and L. L. Raja. Simulation of a DirectCurrent Microdischarge for the Micro Plasma Thruster. IEEE Transactions on Plasma Science, 36(4):1200-1201, 2008.

[40] S. Mahadevan and L. L. Raja, Simulations of Glow Discharge Phenomena in Air for High-Speed Flow Control, AIAA Paper 2008-1093, 2008.

[41] T. Deconinck, S. Mahadevan, and L. L. Raja. Discretization of the Joule heating term for plasma discharge fluid models in unstructured meshes. Journal of Computational Physics, 228(12):4435 - 4443, 2009.

[42] T. Deconinck, S. Mahadevan, and L. L. Raja. Computational simulation of coupled nonequilibrium discharge and compressible flow phenomena in a microplasma thruster. Journal of Applied Physics, 106(6):3305, 2009. 
[43] S. Mahadevan and L. L. Raja. Simulations of direct-current air glow discharge at pressures $~ 1$ Torr: discharge model validation. Journal of Applied Physics, -:-, 2010. accepted for publication.

[44] G. G. Lister. Low-pressure gas discharge modelling. J. Phys. D: Appl. Phys, 25:1649-1680, 1992.

[45] G. J. M. Hagelaar and L. C. Pitchford. Solving the boltzmann equation to obtain electron transport coefficients and rate coefficients for fluid models. Plasma Sources Science and Technology, 14:722-33, 2005.

[46] I. Revel, L. C. Pitchford, and J. P. Boeuf. Calculated gas temperature profiles in argon glow discharges. Journal of Applied Physics, 88(5):2234$2239,2000$.

[47] J. P. Boeuf, L. C. Pitchford, and K. H. Schoenbach. Predicted properties of microhollow cathode discharges in xenon. Applied Physics Letters, 86(7):071501-3, February 2005.

[48] D. L. Scharfetter and H. K. Gummel. Large-signal analysis of a silicon read diode oscillator. Electron Devices, IEEE Transactions on, 16(1):6477, Jan 1969.

[49] S. V. Patankar. Numerical heat transfer and fluid flow. Hemisphere Pub, 1980.

[50] P. S. Kothnur. Simulation of direct current microdischarges for microthruster applications. PhD thesis, The University of Texas at Austin, 2005. 
[51] G. J. M. Hagelaar and G. M. W. Kroesen. Speeding up fluid models for gas discharges by implicit treatment of the electron energy source term. Journal of Computational Physics, 159(1):1-12, 2000.

[52] F. M White. Viscous Fluid Flow. McGraw-Hill, 2nd edition, 1991.

[53] T. J. Barth, A 3D upwind Euler solver for unstructured meshes, AIAA Paper 1991-1548, 1991.

[54] W. K. Anderson and D. L. Bonhaus. An implicit upwind algorithm for computing turbulent flows on unstructured grids. Computers and Fluids, 23(1):1-21, 1994.

[55] V. Venkatakrishnan. Convergence to steady state solutions of the Euler equations on unstructured grids with limiters. Journal of Computational Physics, 118:120-130, 1995.

[56] M-S Liou. A sequel to AUSM: AUSM+. Journal of Computational Physics, 129:364-382, 1996.

[57] A. Haselbacher and J. Blazek. Accurate and efficient discretizations of Navier-Stokes equations on mixed grids. AIAA Journal, 38(11):20942102, November 2000.

[58] V. Venkatakrishnan and D. J Mavriplis. Implicit method for the computation of unsteady flows on unstructured grids. Journal of Computational Physics, 127:380-397, 1996. 
[59] H. Luo, J. D. Baum, and R. Lohner. A fast, matrix-free implicit method for compressible flows on unstructured grids. Journal of Computational Physics, 146(2):664-690, 1998.

[60] H. Luo, J. D. Baum, and R. Lohner. An accurate, fast, matrix-free implicit method for computing unsteady flows on unstructured grids. Computers and Fluids, 30(2):137-159, 2001.

[61] A. V. Phelps and L. C. Pitchford. Anisotropic scattering of electrons by N2 and its effect on electron transport. Physical Review A, 31(5):29322949, May 1985.

[62] D. C. Cartwright, S. Trajmar, A. Chutjian, and W. Williams. Electron impact excitation of the electronic states of N2. II. integral cross sections at incident energies from 10 to $50 \mathrm{eV}$. Physical Review A, 16(3):10411051, 1977.

[63] A. A. Ionin, I. V. Kochetov, A. P. Napartovich, and N. N. Yuryshev. Physics and engineering of singlet delta oxygen production in low-temperature plasma. Journal of Physics D: Applied Physics, 40(2):R25-R61, 2007.

[64] S. A. Lawton and A. V. Phelps. Excitation of the b 1Sigma+ g state of O2 by low energy electrons. Journal of Chemical Physics, 69:1055-1068, 1978.

[65] D. Nelson, M. Benhenni, O. Eichwald, and M. Yousfi. Ion swarm data for electrical discharge modeling in air and flue gas mixtures. Journal of Applied Physics, 94(1):96-103, July 2003. 
[66] A. Bekstein, M. Benhenni, M. Yousfi, O. Ducasse, and O. Eichwald. Ion swarm data of N4+ in N2, O2 and dry air for streamer dynamics simulation. European physical journal. Applied physics : EPJ., 42(1):33-40, 2008.

[67] L. A. Viehland and E. A. Mason. Transport properties of gaseous ions over a wide energy range, IV. Atomic Data and Nuclear Data Tables, $60(1): 37-95,1995$.

[68] R. J. Kee, G. Dixon-Lewis, J. Warnatz, M. E. Coltrin, and J. A. Miller, A fortran computer code package for the evaluation of Gas-Phase multicomponent transport properties, Sandia Report SAND86-8246, 1986.

[69] Y. P. Raizer. Gas Discharge Physics. Springer, 1997.

[70] V. A. Lisovskiy and S. D. Yakovin. Experimental study of a low-pressure glow discharge in air in large-diameter discharge tubes: I. conditions for the normal regime of a glow discharge. Plasma Physics Reports, 26(12):1066-1075, 2000.

[71] T. Deconinck and L. L. Raja. Modeling of mode transition behavior in argon microhollow cathode discharges. Plasma Processes and Polymers, $6(5), 2009$.

[72] A. V. Phelps and Z. L. Petrovic. Cold-cathode discharges and breakdown in argon: surface and gas phase production of secondary electrons. Plasma Sources Science and Technology, 8:R21-R44, 1999. 
[73] A. Fridman. Plasma Chemistry. Cambridge University Press, 2008.

[74] B. Chapman. Glow Discharge Processes: Sputtering and Plasma Etching. John Wiley and Sons, 1981.

[75] W. G. Vincenti and C. H. Kruger. Introduction to physical gas dynamics. Wiley, New York, 1965.

[76] G. Karypis and V. Kumar, METIS: a software package for partitioning unstructured graphs, partitioning meshes, and computing fill-reducing orderings of sparse matrices version 4.0, University of Minnesota, Department of Computer Science, 1998.

[77] J. K. Olthoff. The Gaseous Electronics Conference RF Reference Cell - An Introduction. Journal of Research of the National Institute of Standards and Technology, 100(4), 1995.

[78] E. Meeks, R. S. Larson, P. Ho, C. Apblett, S. M. Han, E. Edelberg, and E. S. Aydil. Modeling of $\mathrm{SiO} 2$ deposition in high density plasma reactors and comparisons of model predictions with experimental measurements. Journal of Vacuum Science \&5 Technology A: Vacuum, Surfaces, and Films, 16(2):544-563, 1998.

[79] Texas Advanced Computing Center HPC Systems. http://www.tacc.utexas. edu/resources/hpc/.

[80] Dell HPC Servers (Poweredge R610 Rack Server). http://content.dell.com/us/en/enterprise/hpcc.aspx. 


\section{VITA}

Shankar Mahadevan graduated from National Public School, Bangalore, India in 2000, and received the B.Tech degree in Aerospace engineering from the Indian Institute of Technology Madras, Chennai, India in 2004. He subsequently joined in the graduate program at the University of Texas at Austin and received the M.S.E degree in Aerospace engineering in December 2006. He enrolled as a Ph.D. student in the spring of 2007.

Permanent address: Aikya, 546, 11th Cross, 4th Main BEL Layout 3rd Block, Vidyaranyapura

Bangalore 560097

India

This Dissertation was typeset with $\mathrm{AT}_{\mathrm{E}} \mathrm{X} 2 \varepsilon^{\dagger}$ by the author.

\footnotetext{
${ }^{\dagger} \mathrm{LT}_{\mathrm{E} X} 2_{\varepsilon}$ is an extension of $\mathrm{H}_{\mathrm{E}} \mathrm{X}$. $\mathrm{HAT}_{\mathrm{E}} \mathrm{X}$ is a document preparation system developed by Leslie Lamport as a special version of Donald Knuth's $\mathrm{T}_{\mathrm{E}} \mathrm{X}$ Program.
} 